\title{
Topological Phase Transitions in Calabi-Yau Compactifications of M-Theory
}

\author{
Dissertation \\ zur Erlangung des akademischen Grades \\ doctor rerum naturalium (Dr. rer. nat.)
}

Vorgelegt dem Rat der Physikalisch-Astronomischen Fakultät der Friedrich-Schiller-Universität Jena

von Dipl.-Phys. Frank Saueressig

geboren am 7. September 1975 in Bad Kreuznach 
Gutachter

1. Dr. habil. Thomas Mohaupt, Jena

2. Prof. Dr. Bernard de Wit, Utrecht

3. Prof. Dr. Jan Louis, Hamburg

Tag der letzten Rigorosumsprüfung: 15. Juni 2004

Tag der öffentlichen Verteidigung: 22. Juni 2004 
Ubi materia - ibi geometria.

Wo Materie ist, da ist Geometrie.

Johannes Kepler 


\section{Contents}

1 Introduction $\quad 1$

$\begin{array}{llr}2 & \text { Background material } & 9\end{array}$

2.1 Five-dimensional gauged supergravity . . . . . . . . . . . . . 9

2.2 Calabi-Yau compactifications . . . . . . . . . . . . 17

2.3 Topological phase transitions . . . . . . . . . . . . . . 18

2.4 Kasner cosmologies . . . . . . . . . . . . . . . 24

3 The hypermultiplet target manifolds $\quad 26$

3.1 Results for the metric and isometries of $X(n-1) \ldots \ldots 28$

3.2 Examples of isometries on $X(2) \ldots \ldots \ldots \ldots$

3.3 The relation to the supergravity conventions . . . . . . . . . 32

4 The flop transition $\quad 34$

4.1 The Lagrangian of the $\mathbb{F}_{1}$-model . . . . . . . . . . . . . . . 34

4.2 The Lagrangian for general flop transitions . . . . . . . . . . . . 39

5 The conifold transition $\quad 46$

5.1 The minimal Lagrangian . . . . . . . . . . . . . . . . . 47

5.2 The Lagrangian for general conifold transitions . . . . . . . . . . . . 51

6 Kasner cosmologies and Out-picture flops $\quad 59$

6.1 General properties of cosmological solutions . . . . . . . . . . 59

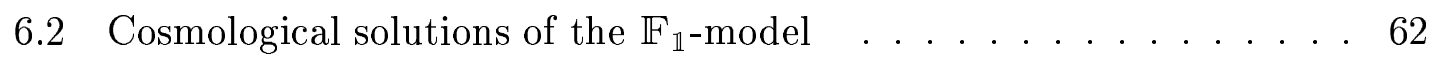

$\begin{array}{lll}7 & \text { Kasner cosmologies and In-picture flops } & 67\end{array}$

7.1 The scalar potential of the $\mathbb{F}_{1}$-model . . . . . . . . . . 67

7.2 Fixed points of the equations of motion . . . . . . . . . 69

7.3 Numerical solutions . . . . . . . . . . . . . . . 73

7.4 The search for inflation . . . . . . . . . . . . . 79

8 Kasner cosmologies and conifold transitions $\quad 84$

8.1 The scalar potential of the minimal Lagrangian . . . . . . . . . 84

8.2 Conifold transitions of cosmological solutions . . . . . . . . . . 86 
9 Summary and Conclusions $\quad 89$

$\begin{array}{ll}\text { References } & 95\end{array}$

$\begin{array}{ll}\text { Publications } & 100\end{array}$

$\begin{array}{ll}\text { A Vector multiplet sector I: one multiplet } & 101\end{array}$

B Vector multiplet sector II: $n_{V}$ multiplets 103

C Vector multiplet sector III: the $\mathbb{F}_{1}$-model 106

D The hypermultiplet sector: $X(n-1) \quad \mathbf{1 1 1}$

D.1 The metrics of the Wolf spaces $X(n-1) \ldots \ldots 111$

D.2 The isometries of the Wolf spaces $X(n-1) \ldots \ldots . \ldots 119$

E Initial values for numerical solutions $\quad 123$

$\begin{array}{ll}\text { Zusammenfassung } & 124\end{array}$

$\begin{array}{ll}\text { Danksagung } & \mathbf{1 3 0}\end{array}$

Ehrenwörtliche Erklärung 132

$\begin{array}{ll}\text { Lebenslauf } & 133\end{array}$ 


\section{Introduction}

Our current understanding of the fundamental interactions occurring in nature is based on the standard model of particle physics and the theory of general relativity. These two theories are tested to very high precision and there is no established discrepancy between their predictions and experimental data. It is, however, widely believed that these are merely effective theories which are only valid below a certain energy scale. This viewpoint is supported by the fact that general relativity cannot be formulated as a consistent quantum field theory, a concept which has been incredibly successful in describing the other fundamental interactions. When quantizing general relativity one faces the problem that the resulting quantum field theory is perturbatively non-renormalizable ${ }^{1}$, i.e., there are ultraviolet divergencies at every order in perturbation theory which destroy the predictive power of the quantum theory. Therefore it is natural to ask if there is a fundamental theory which treats the electromagnetic, strong, and weak interactions and general relativity on the same theoretical footing.

The most prominent candidate for such a unifying theory is string theory $[1,2]$ which, in certain limits, contains both quantum field theory and general relativity. Here the basic idea is to replace the point particles occurring in a quantum field theory by a single one-dimensional extended object, the string. Elementary particles are interpreted as different excitations of this string. These excitations also include a massless symmetric two-tensor which can be identified with the graviton so that string theory naturally incorporates gravity.

When investigating the consequences of this new concept it turns out that there are only five consistent superstring theories which all require a ten-dimensional space-time. These theories possess a very soft ultraviolet behavior at weak string coupling. Here the string scattering amplitudes are exponentially suppressed below

\footnotetext{
${ }^{1}$ There is, however, some evidence that general relativity might be asymptotically safe on the non-perturbative level, see [FS1, FS2, FS6] and references therein.
} 
the string scale, implying that the perturbative expansion is free of ultraviolet divergencies. Further there is a web of non-perturbative dualities which relates the five string theories to one another and also maps their strong coupling regime to dual theories. These dualities suggest that the string theories are different limits of an underlying eleven-dimensional theory called M-theory whose low energy limit is given by the eleven-dimensional supergravity.

Additionally, the non-perturbative sector of these theories contains BPS-solitons called $p$-branes [3]. These are static solutions of the equations of motion which carry electric or magnetic charge with respect to the antisymmetric $(p+1)$-form tensors appearing in the action and extend in $p$ spatial directions. They further satisfy the BPS-bound, i.e., the charge of the solution is (in suitable units) equal to its tension. In particular, the eleven-dimensional supergravity gives rise to an M2- and an M5-brane which are electrically and magnetically charged with respect to the antisymmetric three-form tensor of the action. Especially the M2-brane will play an important role in the following.

To describe a $d$-dimensional universe within string or M-theory one usually makes the ansatz that the ten- or eleven-dimensional space-time decomposes into the (possibly warped) product of a $d$-dimensional observable space-time and a compact internal manifold $X$ whose length scales are below the distances probed by current experiments. The $d$-dimensional physics is conveniently described by a low energy effective action (LEEA). For $X$ being a special holonomy manifold, these LEEA are locally supersymmetric, i.e., $d$-dimensional supergravity actions. These actions usually have a moduli space of vacua, corresponding to the deformations of the internal manifold $X$ and the background fields.

For theories with eight or less supercharges this moduli space includes special points where $X$ becomes singular, leading to a discontinuous or singular LEEA. However, within the full string or M-theory these singularities are believed to be artifacts, which result from ignoring some relevant modes of the theory, namely the 
winding states of strings or branes around the cycles of $X$. Singularities of $X$ arise when such cycles are contracted to zero volume, which gives rise to additional massless states. It was the crucial insight of [4] that the singularities occurring in the LEEA of type II string theory compactified on a Calabi-Yau (CY) threefold with a conifold singularity can be interpreted as arising from illegitimately integrating out such massless states. This has been generalized to many other situations, including M-theory compactifications on CY threefolds [5]. In some cases it is possible to smooth the singularity of $X$ in two or more topologically different ways. This gives rise to so-called topological phase transitions. Such transitions have been studied intensively in literature $[6,7,8,9,5]$. They can be realized as parametric deformations of vacua, but also dynamically $[10,11,12,13]$.

The usual LEEA only include those states which are generically massless, while the extra light modes occurring in a topological phase transition are left out. We refer to this description as the 'Out-picture'. For the complete description of the low energy physics, however, one also needs to include the additional light modes. Following [13], we will call these additional light modes 'transition states'. The low energy description which explicitly includes these states will be referred to as the 'In-picture'.

There are various reasons why it is important to know the In-picture description of topological phase transitions. The compactification of type II string or M-theory on smooth spaces gives rise to a massless spectrum which only contains neutral states. However, in the vicinity of special points one can get non-abelian gauge groups and charged matter $[14,15,16]$, which makes such compactifications viable for particle physics model building. Since in these models all charged particles are transition states, it is clear that one needs the In-picture LEEA to describe their dynamics. It has also been shown that in compactifications with background flux the scalar potential has its minima at special points in moduli space, where additional light states occur $[17,18]$. Conversely, it has been noticed in [13] that 
even without these mechanisms the potential generated by the transition states has the effect of the region in the vicinity of a topological phase transition being dynamically preferred. It was then observed in [19] that including dissipative effects like quantum particle production or Hubble friction may lead to the trapping of the moduli at the transition locus (enhanced symmetry points). Finally, there is some evidence that the interplay between singularities and background flux generates a small scale, which could help to solve the gauge hierarchy and the cosmological constant problem $[20,21,22]$.

Although it is clear in principle that one should be able to "integrate in" the additional states, not much effort has been devoted towards working out the corresponding LEEA explicitly. A systematic investigation was started in [23, 24], by constructing the explicit LEEA which describe $S U(2)$ gauge symmetry enhancement through string or brane winding states in five and four dimensions. For compactifications with 16 supercharges non-abelian gauge symmetry enhancement of the LEEA has been considered in [25].

The first step to obtain analogous results for flop transitions occurring in Mtheory compactified on CY threefolds was made in [13]. In this case the transition states are given by charged hypermultiplets which combine with the neutral hypermultiplets arising from the smooth CY compactification. Local supersymmetry requires that these fields parametrize a non-flat quaternion-Kähler manifold [26]. In [13] the difficulties in working with these rather complicated manifolds were avoided by taking the hypermultiplet manifold to be flat. This, however, is only compatible with global supersymmetry and does not result in a consistent supergravity description of the transition.

The first part of this thesis extends this program and constructs In-picture LEEA for general flop and conifold transitions which are $\mathcal{N}=2$ locally supersymmetric. The strategy is to combine information about the transition states coming from Mtheory with knowledge about the general $\mathcal{N}=2, D=5$ gauged supergravity action 
$[27,28,29,30]$ recently reinvestigated in [31]. ${ }^{2}$ Working in the M-theory setup thereby has the advantage that the vector multiplet sector of the LEEA does not receive quantum corrections and can be determined exactly. Furthermore the moduli cannot bypass the transition locus which in five dimensions is a real codimension one boundary of the vector multiplet moduli space. ${ }^{3}$

As long as the CY threefold $X$ is smooth, the LEEA can be obtained by dimensional reduction [32]. Besides the five-dimensional supergravity multiplet, it contains vector and hypermultiplets whose couplings are determined by $X$. The LEEA is an ungauged supergravity action: all fields are neutral, the gauge group is abelian, and there is no scalar potential. In a flop or conifold transition the Kähler moduli are varied such that $X$ becomes singular through the contraction of $N$ isolated holomorphic curves. The winding states of M2-branes around these curves give rise to $N$ charged hypermultiplets, which become massless at the transition locus [5]. These are the transition states that we want to integrate in. Since they are charged, the resulting action is a gauged supergravity action, which has a non-trivial scalar potential.

The vector multiplet sector of the LEEA contains the Kähler moduli which control the sizes of the $N$ holomorphic curves and, hence, the phase transition. These parametrize a so-called very special real manifold which is completely determined by a cubic polynomial, the prepotential. In the Out-picture the prepotential can be computed exactly and the threshold corrections arising from integrating out the transition states have been derived in [5]. As a result, we can determine the vector multiplet part of the In-picture LEEA exactly.

The situation is much more complicated in the hypermultiplet sector. This is one of the main points addressed this thesis. Local supersymmetry requires that

\footnotetext{
${ }^{2}$ Here $\mathcal{N}$ counts real supercharges in multiples of 4 . Thus $\mathcal{N}=2$ refers to the smallest supersymmetry algebra in five dimensions.

${ }^{3}$ This is in contrast to compactifications of type II string theory where the vector multiplet sector receives non-trivial quantum corrections and the complex vector multiplet scalars need to be fine-tuned in order to run into the transition locus.
} 
the hypermultiplet manifold is a quaternion-Kähler manifold with non-trivial Ricci curvature [26]. The latter constraint excludes hyper-Kähler and in particular flat manifolds. The main difference between (generic) quaternion-Kähler manifolds and other geometries familiar from supersymmetric theories, such as hyper-Kähler and special Kähler manifolds, is that there are no simple, globally defined holomorphic objects which encode the information one needs to construct the LEEA. There is no Kähler potential and in general a quaternion-Kähler manifold is not even a complex manifold. Moreover, for the study of gaugings it would be convenient to take the hypermultiplet manifold to be a direct product, with the neutral fields in one factor and the charged fields in the other. But this is also not an option, because the product of two (generic) quaternion-Kähler manifolds is not quaternion-Kähler.

Due to these complications, this type of geometry is much less understood than the other geometries occurring in supergravity. In particular, only very limited results exist on how to explicitly compute the hypermultiplet metric in string or M-theory. The best studied subsector is the universal hypermultiplet, which at tree level is described by the coset $\frac{U(1,2)}{U(1) \times U(2)}$, but receives non-trivial quantum corrections $[33,34,35]$. The tree level result for the neutral hypermultiplets can be obtained through the c-map [36, 37], but only little is known about quantum corrections (see [38] for a review). Charged multiplets have not been studied at all.

Therefore we take the approach of using a toy model: to describe the hypermultiplet sector of the LEEA we use a particular family of symmetric quaternion-Kähler spaces, the non-compact versions of the unitary Wolf spaces

$$
X(1+N)=\frac{U(1+N, 2)}{U(1+N) \times U(2)}
$$

containing $N+1$ hypermultiplets. In order to cope with the technical problems arising in the hypermultiplet sector it is extremely useful that every quaternion-Kähler manifold can be obtained from a so-called hyper-Kähler cone by a superconformal quotient [39]. As indicated by the name, this construction is intimately related to 
the construction of hypermultiplet actions using the superconformal tensor calculus $[40,41,42]$. This treatment does not utilize the fact that the spaces $X(1+N)$ happen to be Kähler, but only relies on techniques which apply to any quaternionKähler manifold. Moreover, working on the level of the hyper-Kähler cone has the advantage that the product of two hyper-Kähler cones is again a hyper-Kähler cone. Thus one can put the neutral and charged fields in separate factors. The isometries of $X(1+N)$ are also obtained from their counterparts on the corresponding hyperKähler cone. We find the resulting parametrization very useful for discussing the gauging of the LEEA, as there is a simple relation between the microscopic data and the Killing vectors occurring in the LEEA. The parametrization of $X(1+N)$ used in [37], which relies on its Kähler structure, is much less useful for this.

In the second part of this thesis we use our In-picture LEEA to investigate the effect of the dynamical transition states on five-dimensional Kasner cosmological solutions. ${ }^{4}$ We thereby extend earlier work by Brändle and Lukas [13] on flop transitions who addressed this question using a non-supersymmetric effective action. Even though these five-dimensional cosmologies are not directly related to our observable universe, there are nevertheless two relevant questions which can also be addressed in this five-dimensional setup.

First, studying cosmological solutions with time-dependent moduli allows to explicitly address the question of dynamical moduli stabilization. As these moduli parametrize the shape and volume of the internal manifold this is equivalent to asking whether there are manifolds $X$ which are dynamically preferred. In particular the time-dependence of the volume of $X$ is of importance, as a rapid decompactification may not be compatible with experimental observations.

Second, the last couple of years have seen a tremendous improvement in measuring cosmological observables [44, 45, 46]. This data supports a 'cosmological standard scenario' [47] which includes a period of primordial inflation $[48,49]$ in

\footnotetext{
${ }^{4}$ For a review of these cosmologies in the context of string theory see [43].
} 
the early universe. Further, there is some evidence that the modest acceleration observed today is only a very recent phenomenon in the history of the universe [50]. While there is a wealth of models explaining this observational input by postulating an 'ad-hoc' scalar potential, it turns out that deriving such potentials from string or M-theory is rather hard. Even though there has been considerable progress in this direction $[51,52,53]$, inflation is non-generic and involves some degree of 'functional fine tuning'. Subsequently it was argued in $[54,55]$ that realistic cosmologies will most likely occur in the 'central region of moduli space' which is not accessible by string dualities.

The inclusion of transition states in the LEEA thereby provides a natural way to obtain scalar potentials from string or M-theory compactifications. As we will see in the following, these potentials are completely fixed by the underlying M-theory data, so that there is no room for fine-tuning. They also provide explicit examples for potentials arising in the central region of moduli space, leading to the natural question if they have the necessary properties to support inflation. In particular the potentials constructed in this thesis have both flat and steep directions which makes it concievable that they give rise to hybrid inflation $[56,57]$. This would in particular be interesting in the context of brane world scenarios [58] where the four-dimensional universe is embedded into a higher-dimensional space-time.

The remaining parts of this thesis are organized as follows. In section 2 we review the necessary background material on five-dimensional supergravity, CY compactifications, topological phase transitions, and Kasner cosmologies. The superconformal quotient construction of the metrics, the Killing vectors and the moment maps of the Wolf spaces $X(N+1)$ is carried out in the appendix D and our results are summarized in section 3. These are used to construct the In-picture LEEA describing general flop and conifold transitions in sections 4 and 5, respectively. In sections 6 and 7 we investigate the properties of Kasner cosmologies undergoing a flop transition in the Out- and the In-picture. The corresponding analysis for 
conifold transitions is given in section 8 . We conclude with a summary in section 9 .

Some of the results presented in this thesis have already been published. The superconformal quotient construction of the geometrical data for the Wolf spaces $X(N+1)$ and the In-picture LEEA for flop transitions appeared in [FS3]. The Kasner cosmological solutions given in sections 6 and 7 were discussed in [FS4, FS5] which also include some additional material. The results on conifold transitions in sections 5 and 8 will appear in a future publication [FS10]. Additional results on accelerating cosmologies arising from M-theory compactified on a maximally symmetric internal space and on the interplay between space-time singularities and the Kähler cone have been published in refs. [FS8] and [FS9], respectively, but are not included in this thesis. The same applies to the results on supersymmetric Euclidean actions obtained in [FS7].

\section{Background material}

We begin with a review of the relevant properties of $\mathcal{N}=2, D=5$ gauged supergravity $[27,28,29,30,31]$ and its relation to M-theory compactified on CY threefolds. For smooth CY compactifications this relation was worked out in [32]. Our conventions for the five-dimensional gauged supergravity action follow [59]. We refer to these papers for further details.

\subsection{Five-dimensional gauged supergravity}

The LEEA of eleven-dimensional supergravity compactified on a smooth CY threefold $X$ with Hodge numbers $h^{p, q}$ is given by five-dimensional supergravity coupled to $n_{V}=h^{1,1}-1$ abelian vector and $n_{N}=h^{2,1}+1$ neutral hypermultiplets. By explicitly including the transition states arising in a flop or conifold transition we additionally obtain $\delta n_{H}$ charged hypermultiplets. 
The natural starting point for the construction of a LEEA which includes these states is given by the general $\mathcal{N}=2, D=5$ gauged supergravity action with $n_{V}$ vector, $n_{H}=n_{N}+\delta n_{H}$ hyper and no tensor multiplets. Anticipating the results of the subsequent sections, we limit ourselves to the case of abelian gaugings. The bosonic matter content of this theory consists of the graviton $e_{\mu}^{a}, n_{V}+1$ vector fields $A_{\mu}^{I}$ with field strength $F_{\mu \nu}^{I}=\partial_{\mu} A_{\nu}^{I}-\partial_{\nu} A_{\mu}^{I}, n_{V}$ real vector multiplet scalars $\phi^{x}$, and $4 n_{H}$ real hypermultiplet scalars $q^{X}$. The bosonic part of the Lagrangian reads:

$$
\begin{aligned}
\sqrt{-g}^{-1} \mathcal{L}_{\text {bosonic }}^{\mathcal{N}=2}= & -\frac{1}{2} R-\frac{1}{4} a_{I J} F_{\mu \nu}^{I} F^{J \mu \nu} \\
& -\frac{1}{2} g_{X Y} \mathcal{D}_{\mu} q^{X} \mathcal{D}^{\mu} q^{Y}-\frac{1}{2} g_{x y} \mathcal{D}_{\mu} \phi^{x} \mathcal{D}^{\mu} \phi^{y} \\
& +\frac{1}{6 \sqrt{6}} C_{I J K} \sqrt{-g}^{-1} \epsilon^{\mu \nu \rho \sigma \tau} F_{\mu \nu}^{I} F_{\rho \sigma}^{J} A_{\tau}^{K}-\mathrm{g}^{2} \mathbb{V}(\phi, q) .
\end{aligned}
$$

The scalars $\phi^{x}$ and $q^{X}$ parametrize a very special real manifold $\mathcal{M}_{\mathrm{VM}}$ [27] and a quaternion-Kähler ${ }^{5}$ manifold $\mathcal{M}_{\mathrm{HM}}[26]$. The manifolds $\mathcal{M}_{\mathrm{HM}}$ are Einstein. A ghostfree action requires that their Ricci scalar satisfies $\mathcal{R}=-8 n_{H}\left(n_{H}+2\right)$ which is readily seen using the superconformal calculus [60].

The vector multiplet sector is determined by the completely symmetric tensor $C_{I J K}$, appearing in the Chern-Simons term. This tensor is used to define a real homogeneous cubic polynomial

$$
\mathcal{V}(h)=C_{I J K} h^{I} h^{J} h^{K}
$$

in $n_{V}+1$ real variables $h^{I}$. The $n_{V}$-dimensional manifold $\mathcal{M}_{\mathrm{VM}}$ is obtained by restricting this polynomial to the hypersurface

$$
\mathcal{V}(\phi)=C_{I J K} h^{I}(\phi) h^{J}(\phi) h^{K}(\phi)=1
$$

\footnotetext{
${ }^{5}$ In parts of the physics literature, including [26], these manifolds are called 'quaternionic'. However, in the mathematical literature quaternionic is a weaker condition than 'quaternion(ic)Kähler'. Definitions for both kinds of manifolds are given later in the main text.
} 
The coefficients $a_{I J}$ appearing in the kinetic term of the vector field strength are given by

$$
\begin{aligned}
a_{I J}(h) & :=-\left.\frac{1}{3} \frac{\partial}{\partial h^{I}} \frac{\partial}{\partial h^{J}} \ln \mathcal{V}(h)\right|_{\mathcal{V}=1} \\
& =-2 C_{I J K} h^{K}+3 C_{I K L} C_{J M N} h^{K} h^{L} h^{M} h^{N} .
\end{aligned}
$$

Defining

$$
h_{x}^{I}:=-\sqrt{\frac{3}{2}} \frac{\partial}{\partial \phi^{x}} h^{I}(\phi), \quad h_{I}:=C_{I J K} h^{J} h^{K},
$$

the metric on $\mathcal{M}_{\mathrm{VM}}$ is proportional to the pullback ${ }^{6}$ of $a_{I J}$,

$$
g_{x y}(\phi):=h_{x}^{I} h_{y}^{J} a_{I J}
$$

The hypermultiplet scalars $q^{X}$ parametrize a quaternion-Kähler manifold of dimension $\operatorname{dim}_{\mathbb{R}}\left(\mathcal{M}_{\mathrm{HM}}\right)=4 n_{H}$. For $n_{H}>1$ such manifolds are characterized by their holonomy group,

$$
\operatorname{Hol}\left(\mathcal{M}_{\mathrm{HM}}\right)=S U(2) \cdot U S p\left(2 n_{H}\right)
$$

while in the case $n_{H}=1$ they are defined as Einstein spaces with self-dual Weyl curvature. The restricted holonomy group implies that the curvature tensor decomposes into an $S U(2)$ and $U S p\left(2 n_{H}\right)$ part

$$
R_{X Y W Z} f_{i A}^{W} f_{j B}^{Z}=\epsilon_{i j} R_{X Y A B}+C_{A B} R_{X Y i j}
$$

Here $i=1,2$ is an $S U(2)$ index and $A=1, \ldots, 2 n_{H}$ is a $U S p\left(2 n_{H}\right)$ index. These are raised and lowered by the symplectic metrics $\epsilon_{i j}$ and $C_{A B}$, respectively. The $4 n_{H}$-bein $f_{X}^{i A}$ is related to the metric on $\mathcal{M}_{\mathrm{HM}}$ by

$$
g_{X Y}=f_{X}^{i A} f_{Y}^{j B} \epsilon_{i j} C_{A B}=f_{X}^{i A} f_{Y i A}
$$

\footnotetext{
${ }^{6}$ The $a_{I J}$ can be interpreted as a metric on the space into which $\mathcal{M}_{\mathrm{VM}}$ is immersed by $(2.3)$.
} 
and satisfies:

$$
f_{i A}^{X} f_{Y}^{i A}=\delta_{Y}^{X}, \quad f_{i A}^{X} f_{X}^{j B}=\delta_{i}{ }^{j} \delta_{A}{ }^{B} .
$$

Local supersymmetry requires the $S U(2)$ part of the curvature to be non-vanishing [26]. This feature excludes hyper-Kähler manifolds as target manifolds, since these have trivial $S U(2)$ curvature.

The superconformal quotient construction $[40,41]$ employed in the next section provides a method to obtain all the quantities of interest in the hypermultiplet sector. In this approach the metric $g_{X Y}$ and all its isometries are computed from their counterparts on the associated hyper-Kähler cone, without the need to introduce the vielbein $f_{X}^{i A}$. However, to be able to relate our results to the major part of the literature on hypermultiplets, we review the properties of quaternion-Kähler manifolds using the vielbein $f_{X}^{i A}$.

We first introduce the Levi-Civita connection $\Gamma_{Y Z}{ }^{X}$, a $U S p\left(2 n_{H}\right)$ connection $\omega_{X B}{ }^{A}$, and an $S U(2)$ connection $\omega_{X k}{ }^{i}$. The vielbein is covariantly constant with respect to these connections,

$$
\partial_{X} f_{Y}^{i A}-\Gamma_{X Y}^{Z} f_{Z}^{i A}+f_{Y}^{i B} \omega_{X B}^{A}+\omega_{X k}^{i} f_{Y}^{k A}=0
$$

The $S U(2)$ curvature can be expressed in terms of the vielbein as

$$
\mathcal{R}_{X Y i j}=f_{X C(i} f_{j) Y}^{C}
$$

Raising the index $j$ with $\epsilon^{i j}$, we can expand the $S U(2)$ curvature in terms of the standard Pauli matrices,

$$
\mathcal{R}_{X Y i}{ }^{j}=i \mathcal{R}_{X Y}^{r}\left(\sigma_{r}\right)_{i}{ }^{j}
$$

where $r=1,2,3$ enumerates the Pauli matrices. The $\mathcal{R}_{X Y}^{r}$ defined in this way are 
real and satisfy

$$
\mathcal{R}_{X Y}^{r} \mathcal{R}^{s Y Z}=-\frac{1}{4} \delta^{r s} \delta_{X}{ }^{Z}-\frac{1}{2} \epsilon^{r s t} \mathcal{R}_{X}^{t}{ }^{Z}
$$

It is no accident that the above formula resembles the quaternionic algebra. A quaternion-Kähler manifold is in particular quaternionic, i.e., there locally exists a triplet of almost complex structures, which satisfy the quaternionic algebra. The curvatures $\mathcal{R}_{X Y}^{r}$ are proportional to the Kähler forms derived from these almost complex structures. However, since in general none of these almost complex structures is integrable, a quaternion-Kähler manifold does not need to be Kähler, or, in fact, not even be complex.

We now turn to the isometries of $\mathcal{M}_{\mathrm{HM}}$ which are relevant for the gauging. These must be compatible with the three locally defined almost complex structures, i.e., they leave the almost complex structures invariant up to an $S U(2)$ rotation. Such isometries are called tri-holomorphic. Given a tri-holomorphic Killing vector $K_{I}^{X}(q)$ on the quaternion-Kähler manifold, the $\mathcal{R}_{X Y}^{r}$ can be used to construct an $S U(2)$ triplet of real prepotentials $P_{I}^{r}(q)$, the so-called moment maps [61]:7

$$
\mathcal{R}_{X Y}^{r} K_{I}^{Y}=D_{X} P_{I}^{r}, \quad D_{X} P_{I}^{r}:=\partial_{x} P_{I}^{r}+2 \epsilon^{r s t} \omega_{X}^{s} P_{I}^{t}
$$

Here $\omega_{X}^{s}$ is defined by $\omega_{X i}{ }^{j}=: i \omega_{X}^{r}\left(\sigma_{r}\right)_{i}{ }^{j}$. Using eq. (2.14) this relation can be solved for the Killing vector $K_{I}^{Y}(q)$ :

$$
K_{I}^{Z}=-\frac{4}{3} \mathcal{R}^{r}{ }^{Z X} D_{X} P_{I}^{r}
$$

Hence the moment map $P_{I}^{r}$ provides a triplet of functions from which the Killing vectors of $\mathcal{M}_{\mathrm{HM}}$ can be obtained. Additionally, one can show that eq. (2.15) determines the prepotentials uniquely. In particular, covariantly constant shifts $P_{I}^{r(0)}$ are excluded. This is shown by first contracting eq. (2.15) with $D_{X}$ and then using the

\footnotetext{
${ }^{7} \mathrm{Up}$ to a rescaling, these are identical to the $\hat{\mu}^{r}$ given in the next section.
} 
harmonicity property of the prepotentials [62]:

$$
P_{I}^{r}=\frac{1}{2 n_{H}} D_{X}\left(K_{I Y} \mathcal{R}^{r X Y}\right)
$$

By virtue of eq. (2.15), this relation implies $P_{I}^{r(0)}=0$ as $\mathcal{R}_{X Y}^{r} K_{I}^{Y}=0$ for a covariantly constant shift. Hence there is no analog of $D=4, \mathcal{N}=1$ Fayet-Iliopoulos terms in $D=5, \mathcal{N}=2$ supergravity with a non-trivial hypermultiplet sector.

We now discuss the gauging of the Lagrangian (2.1). The scalars take values in a Riemannian manifold, and the gauge group must operate on them as a subgroup of the isometry group in order to keep the action invariant. This procedure includes the covariantization of the derivatives appearing in the scalar kinetic terms with respect to isometries of the vector or hypermultiplet target manifolds,

$$
\mathcal{D}_{\mu} q^{X}:=\partial_{\mu} q^{X}+\mathrm{g} A_{\mu}^{I} K_{I}^{X}(q), \quad \mathcal{D}_{\mu} \phi^{x}:=\partial_{\mu} \phi^{x}+\mathrm{g} A_{\mu}^{I} K_{I}^{x}(\phi)
$$

Here the $K_{I}^{X}(q)$ and $K_{I}^{x}(\phi)$ are the Killing vectors of the gauged isometries in the hypermultiplet and vector multiplet scalar manifold, respectively. An important consequence of the gauging is that we now have a non-trivial scalar potential $\mathbb{V}(\phi, q)$. Since we have both vector and hypermultiplets but no tensor multiplets, this potential is determined by the gauging of the hypermultiplet isometries. In order to write down $\mathbb{V}$ explicitly, we define:

$$
P^{r}(\phi, q):=h^{I}(\phi) P_{I}^{r}(q), \quad P_{x}^{r}(\phi, q):=h_{x}^{I}(\phi) P_{I}^{r}(q), \quad K^{X}(\phi, q):=h^{I}(\phi) K_{I}^{X}(q)
$$

Here $h^{I}(\phi)$ are the scalars $(2.2)$ associated to the gauge field $A_{\mu}^{I}, K_{I}^{X}(q)$ denotes the Killing vector of the hypermultiplet isometry for which $A_{\mu}^{I}$ serves as a gauge connection, and $P_{I}^{r}$ is its associated $S U(2)$ triplet of moment maps. The scalar 
potential takes the form

$$
\mathbb{V}(\phi, q)=-4 P^{r} P^{r}+2 g^{x y} P_{x}^{r} P_{y}^{r}+\frac{3}{4} g_{X Y} K^{X} K^{Y}
$$

To discuss the vacuum structure of this potential, it is further useful to introduce the real 'superpotential' [59]

$$
\mathcal{W}:=\sqrt{\frac{2}{3} P^{r} P^{r}}
$$

which can be read off from the supersymmetry variations of the gravitino. Under the condition that the phase $Q^{r}$ defined by

$$
P^{r}=\sqrt{\frac{3}{2}} \mathcal{W} Q^{r}, \quad Q^{r} Q^{r}=1
$$

is independent of the vector multiplet scalars, $\partial_{x} Q^{r}=0$, the scalar potential $(2.20)$ can be rewritten in terms of $\mathcal{W}$ :

$$
\mathbb{V}(\phi, q)=-6 \mathcal{W}^{2}+\frac{9}{2} g^{\Lambda \Sigma} \partial_{\Lambda} \mathcal{W} \partial_{\Sigma} \mathcal{W}
$$

Here $\phi^{\Lambda}, \Lambda, \Sigma=1, \ldots n_{V}+4 n_{H}$ denotes the combined set of vector and hypermultiplet scalar fields and $g^{\Lambda \Sigma}$ is the direct sum of the vector and hypermultiplet inverse metrics,

$$
g^{\Lambda \Sigma}(\phi, q):=g^{X Y}(q) \oplus g^{x y}(\phi)
$$

The 'stability form' (2.23) is useful, because it is sufficient to guarantee the gravitational stability of the theory [63].

It was further shown in $[59,64]$ that the minima of $\mathcal{W}$ are given by the solution 
of the algebraic equations ${ }^{8}$

$$
h_{I}\left(\phi_{c}\right) P^{r}\left(\phi_{c}, q_{c}\right)=P_{I}^{r}\left(q_{c}\right) \text { and } K^{X}\left(\phi_{c}, q_{c}\right)=0 .
$$

Here $\phi_{c}$ and $q_{c}$ denote the submanifolds of the vector and hypermultiplet scalar manifolds where the conditions (2.25) are satisfied. These submanifolds correspond to the supersymmetric vacua of the theory. Indeed it is straightforward to check that the solutions of the equations (2.25) are critical points of the potential (2.20) with $\left.\mathbb{V}\right|_{\phi_{c}, q_{c}} \leq 0$, implying that the resulting vacua are Minkowski or anti-de Sitter. Further $\partial_{x} Q^{r}=0$ is always satisfied on the submanifolds $\phi_{c}, q_{c}$ so that $\mathbb{V}$ restricted to these subspaces can always be rewritten in its stability form (2.23).

Depending on the choice of gauging there are several types of solutions of this equations:

- There are no solutions at all. In this case the entire supersymmetry is broken spontaneously.

- There are isolated supersymmetric solutions. This implies that all moduli of the theory are fixed and there are no flat directions in the potential.

- There may be solutions where the conditions (2.25) fix (some of) the hypermultiplet scalars, while the vector multiplet scalars are unconstrained. This is called a Coulomb branch.

- On the contrary there can be solutions where (some of) the vector multiplet scalars are fixed while the hypermultiplet scalars are free to take values in a submanifold of $\mathcal{M}_{\mathrm{HM}}$. This corresponds to a Higgs branch.

- Finally there can also be solutions where both vector and hypermultiplet scalars are restricted. This is called a mixed branch.

\footnotetext{
${ }^{8}$ For analogous relations in $\mathcal{N}=2, D=4$ super-Yang-Mills theory see [65].
} 
As it will turn out, the vacua of the Lagrangians constructed in this thesis correspond to either Coulomb or Higgs branches, and the other cases will not be relevant in the following.

\subsection{Calabi-Yau compactifications}

When compactifying eleven-dimensional supergravity on a smooth CY threefold $X$ [32], one obtains a five-dimensional ungauged supergravity action, i.e, all fields are neutral under the gauge group $U(1)^{n_{V}+1}$ and there is no scalar potential. In this case the objects introduced above acquire a geometrical interpretation: the vector multiplet scalars encode the deformations of the Kähler class of $X$ at fixed total volume, while the hypermultiplet scalars parametrize the volume of $X$, deformations of its complex structure, and deformations of the three-form gauge field. The hypermultiplet containing the volume is called the universal hypermultiplet, because it is insensitive to the complex structure of $X$. Further, the $C_{I J K}$ determining the vector multiplet sector of the LEEA are given by the triple intersection numbers of $X$,

$$
C_{I J K}=D_{I} \cdot D_{J} \cdot D_{K}
$$

where the $D_{I}, I=0, \ldots, n_{V}$ are a basis of the homological four-cycles $H_{4}(X, \mathbb{Z})$. The dual basis $C^{I}$ for the two-cycles is defined by

$$
C^{I} \cdot D_{J}=\delta^{I}
$$

By integrating the Kähler form $J$ over the two-cycles $C^{I}$ we obtain the quantities

$$
\hat{h}^{I}=\int_{C^{I}} J,
$$


which control the volumes of even-dimensional cycles of $X$. In particular, the overall volume of $X$ is given by

$$
\operatorname{vol}(X)=\frac{1}{3 !} \int_{X} J \wedge J \wedge J=\frac{1}{6} C_{I J K} \hat{h}^{I} \hat{h}^{J} \hat{h}^{K}
$$

Since the modulus corresponding to the total volume belongs to the universal hypermultiplet, one needs to introduce rescaled fields

$$
h^{I}=(6 \operatorname{vol}(X))^{-1 / 3} \hat{h}^{I},
$$

in order to separate the vector and hypermultiplet moduli $[32,13]$. These rescaled moduli appear in the cubic polynomial (2.3). Moreover, one needs to split the volume into the volume modulus $\mathrm{V}$, which is a dynamical field, and a fixed reference volume $\mathrm{v}$, which relates the eleven-dimensional and the five-dimensional gravitational couplings,

$$
\operatorname{vol}(X)=\mathrm{v} \cdot \mathrm{V}, \quad \text { where } \frac{\mathrm{v}}{\kappa_{(11)}^{2}}=\frac{1}{\kappa_{(5)}^{2}}
$$

\subsection{Topological phase transitions}

We now review the geometry and M-theory physics of the topological phase transitions which will be investigated in this thesis. The discussion follows $[6,5,66]$ while additional background information can be found in $[67,8]$.

The Kähler moduli space of a CY threefold $X$ is a cone, called the Kähler cone. This cone is defined by the condition that the volumes of all curves $C \subset X$, holomorphic surfaces $S \subset X$, and $X$ itself are positive when measured with the Kähler form $J$

$$
\int_{C} J>0, \quad \int_{D} J \wedge J>0 \quad, \quad \int_{X} J \wedge J \wedge J>0
$$


The vector multiplet moduli space is the projectivization of this cone, or, equivalently, a hypersurface corresponding to fixed total volume. At the boundaries of the Kähler cone some submanifolds of $X$ contract to zero volume and $X$ becomes a singular CY space $\hat{X}$. If there is a second, inequivalent, way to smooth the singularities of $\hat{X}$, leading to a topologically different CY manifold $\tilde{X}$, then $X$ and $\tilde{X}$ are said to be related by a topological phase transition.

In this thesis we are interested in one particular type of singular CY spaces $\hat{X}$, namely those containing $N$ isolated nodes (double points). These occur if $N$ isolated holomorphic curves $\mathcal{C}_{i}, i=1, \ldots, N$, have collapsed to points. Locally these nodes can be described as the vertex of a cone in $\mathbb{C}^{4}$ given by the zero locus of a quadratic polynomial. One particular way to parametrize this cone is the zero locus of the polynomial $\Psi=x y-z t$ where $x, y, z, t$ are the complex coordinates of $\mathbb{C}^{4}$. The base of this complex cone is given by $\mathbb{P}^{1} \times \mathbb{P}^{\prime 1}$ where $\mathbb{P}^{n}$ denotes the complex projective space of complex dimension $n$. The nodes in $\hat{X}$ can be resolved by expanding them into one of the $\mathbb{P}^{1}$ 's. This is called a small resolution. Alternatively the cone can locally be described as the zero locus of the polynomial $\Psi=\sum_{i=1}^{4} \zeta_{i}^{2}$ with the $\zeta_{i}$ again being complex coordinates in $\mathbb{C}^{4}$. In this case the base of the real cone is given by the product $S^{2} \times S^{3}$ where the $S^{2} \simeq \mathbb{P}^{1}$ is identified with one of the $\mathbb{P}^{1}$ s introduced before. This indicates that besides resolving the node by a small resolution, the singularity can also be expanded into a three-sphere. This is called a deformation. ${ }^{9}$

While smoothing the nodes of $\hat{X}$ by either small resolutions or deformations results in a smooth manifold, the condition that this manifold is again CY places some global constraints on the smoothing. In fact the $N$ node case has only two types of topological phase transitions connecting different CY manifolds $X$ and $\tilde{X}$.

If the $N$ contracting holomorphic curves $\mathcal{C}_{i}$ belong to the same homology class

\footnotetext{
${ }^{9}$ In principle the node can also be expanded into a surface of complex codimension one which is called a blow-up. This, however, is not compatible with the resulting smoothed manifold being CY.
} 
$C^{\star}=q_{I} C^{I}$ and have volume $h^{\star}=q_{I} h^{I}$ there is the possibility of a flop transition. ${ }^{10}$ In this case the cycles $\mathcal{C}_{i}$ (which correspond to the $\mathbb{P}^{1}$ in the base of each cone, say) are contracted and the resulting nodes are resolved into holomorphic curves $\tilde{\mathcal{C}}_{i}$ (which correspond to the $\mathbb{P}^{\prime 1}$ in the base of the cones) in the homology class $\tilde{C}^{\star}=-C^{\star}$. This operation relates two topological different but birationally equivalent CY manifolds $X$ and $\tilde{X}$. The Kähler cones of $X$ and $\tilde{X}$ can be glued together along their common boundary $h^{\star}=0$. The triple intersection numbers $\tilde{C}_{I J K}$ of $\tilde{X}$ are related to those of $X$ by

$$
\tilde{C}_{I J K}=C_{I J K}-\frac{N}{6}\left(D_{I} \cdot C^{\star}\right)\left(D_{J} \cdot C^{\star}\right)\left(D_{K} \cdot C^{\star}\right)
$$

while the Hodge and Euler numbers do not change in the transition. By joining the Kähler cones of all CY threefolds related by flops, one obtains the extended Kähler cone.

The second transition can occur if the sum of the $N$ collapsing cycles $\mathcal{C}_{i}$ of $X$ is homologically trivial and satisfies $N>r>0$ homology relations

$$
\sum_{i=1}^{N} a_{\rho i}\left[\mathcal{C}_{i}\right]=0, \quad \rho=1, \ldots, r
$$

In this case we have $N-r$ independent cycles whose contraction results in $N$ nodes. Deforming these nodes into three-spheres then also leads to a topological different CY manifold $\tilde{X}$ and is called a conifold transition. The Hodge numbers of $X$ and $\tilde{X}$ are related by

$$
h^{1,1}(\tilde{X})=h^{1,1}(X)-(N-r), \quad h^{1,2}(\tilde{X})=h^{1,2}(X)+r
$$

\footnotetext{
${ }^{10}$ In the language of Wilson [67] this is a type-I contraction. Type-III contractions which correspond to a four-cycle collapsing to a two-cycle of genus $g$ and lead to $S U(2)$-enhancement (i.e., additional massless vector multiplets) have been studied in $[23,24]$ and will not be considered here. Further there are type-II contractions where a four-cycle collapses to a point and the cubic cone where the CY threefold degenerates. For these cases no supergravity description is known and they will also not be considered here.
} 
implying a change in the Euler characteristic $\chi_{\mathrm{E}}(\tilde{X})=\chi_{\mathrm{E}}(X)-2 N$. We also note that the nodes of a conifold transition cannot be deformed in topologically different ways. Even though the deformation contains an entire $S O(2)$-family of three-spheres, these are all homotopically equivalent.

In terms of the microscopic M-theory these transitions have the following interpretation. In the smooth $\mathrm{CY}$ threefold $X$ the holomorphic curves $\mathcal{C}_{i}$ can be expanded in the homology basis, $C^{i}=q_{I}^{i} C^{I}$, and have a volume $h^{i}=q^{i}{ }_{I} h^{I}$. By wrapping M2branes around these curves, one obtains $N$ hypermultiplets ${ }^{11}$ which carry charges $\pm\left(q_{I}^{i}\right), I=0, \ldots, n_{V}$, under the gauge fields $A_{\mu}^{I}$. This means that each of these multiplets is charged under the gauge group $U(1) \subset U(1)^{n_{V}+1}$ associated with the gauge field $A_{\mu}^{i}=q_{I}^{i} A_{\mu}^{I}$. Their masses are proportional to the volume of the holomorphic curves. By dimensional reduction of the M2-brane action one computes the mass $[13]$

$$
M^{i}=T_{(2)}(6 \mathrm{v})^{1 / 3}\left|q_{I}^{i} h^{I}\right|
$$

where $T_{(2)}$ is the tension of the M2-brane. Since $Z^{i}= \pm q^{i}{ }_{I} h^{I}$ is the central charge of the charged states with respect to the five-dimensional supersymmetry algebra, we recognize the five-dimensional BPS mass formula $M_{(\mathrm{BPS})}^{i}=$ const $\cdot\left|Z^{i}\right| .^{12}$ As these states become massless at $h^{i}=0$, it is precisely these charges hypermultiplets which we want to include in our In-picture LEEA.

In the case of a conifold transition the nodes arising from the collapsing cycles $\mathcal{C}_{i}$ can also be deformed into three-cycles of the CY threefold $\tilde{X}$. The corresponding change in the Hodge numbers (2.35) indicates that the number of massless vector and hypermultiplets on the two sides of the transition is related by $\tilde{n}_{V}=n_{V}-(N-r)$

\footnotetext{
${ }^{11}$ Recall that a hypermultiplet is a short representation of the supersymmetry algebra where half of the supersymmetry generators act trivially. The mass of such short representations needs to saturate the BPS bound, i.e., the mass is (in suitable units) equal to the central charge carried by the multiplet.

${ }^{12}$ From the eleven-dimensional point of view the mass of a wrapped M2-brane is given by $M_{(11)}=$ $T_{(2)} \operatorname{vol}(\mathcal{C})=T_{(2)}(6 \mathrm{vV})^{1 / 3}\left|q_{I} h^{I}\right|$. However, the relation between the eleven-dimensional and fivedimensional metrics involves a conformal rescaling by the volume modulus $\mathrm{V}$.
} 
and $\tilde{n}_{N}=n_{N}+r$, respectively. Including the $N$ additional hypermultiplets which are massless at the transition locus, the massive states on the $\tilde{X}$ branch should be given by the degrees of freedom associated with $N-r$ vector and hypermultiplets. But as both vector and hypermultiplets are short multiplets whose mass must be equal to their central charge, one expects that these degrees of freedom organize themselves into long representations of the supersymmetry algebra. Investigating these representations [68] yields that this can be achieved by a vector multiplet 'eating up' a hypermultiplet to form a massive long vector multiplet. Therefore we expect that the transition states on the $\tilde{X}$ branch are given by $N-r$ long vector multiplets which become massless at the transition locus. The non-BPS nature of these multiplets thereby fits nicely with the fact that the microscopic picture does not contain BPS-branes which could wrap three-cycles to give rise to pointlike BPS-states. ${ }^{13}$

In the case of the flop it was shown in [5] that the discontinuity in the triple intersection numbers indicated by (2.33) can be interpreted as a threshold correction arising from illegitimately integrating out the charged hypermultiplets which become massless at the transition locus. Our aim now is to explicitly include these states in the LEEA, then called the In-picture LEEA $\hat{S}$, to obtain a smooth description of the physics at the transition locus. In the language of Wilsonian effective actions the relation between the Out- and the In-picture is as follows. The collapsing cycles $\mathcal{C}_{i}$ give rise to $N$ charged hypermultiplets with mass proportional to $h^{i}$. For energy scales below these masses, we can use the standard LEEA $S$, obtained by the dimensional reduction on $X$ whose vector multiplet sector is determined by the prepotential $\mathcal{V}$. However, the complete low energy description in the vicinity of the transition locus requires that we work with the In-picture LEEA $\hat{S}$. The vector multiplet sector of $\hat{S}$ is completely determined by the prepotential $\hat{\mathcal{V}}$, which

\footnotetext{
${ }^{13}$ The exact microscopic origin of these degrees of freedom remains to be clarified. For the case of type IIB string theory it was argued in [7] that these states correspond to massive string excitations.
} 
we would like to determine in terms of $\mathcal{V}$. To find this relation we note that there is an intermediate regime, where the transition states have small, but non-vanishing masses. Here both the actions $\hat{S}$ and $S$ are valid. Therefore one can relate $\hat{S}$ to $S$ by integrating out the transition states. In the vector multiplet sector this can be done exactly. Using the result of [5] we obtain ${ }^{14}$

$$
\hat{\mathcal{V}}=\mathcal{V}-\frac{1}{2} \sum_{i=1}^{N}\left(h^{i}\right)^{3}
$$

For flop transitions this result can be brought to a suggestive form by writing the $\hat{C}_{I J K}$ as 'averaged triple intersection numbers' [23],

$$
\hat{C}_{I J K}=\frac{1}{2}\left(C_{I J K}+\tilde{C}_{I J K}\right)
$$

where $C_{I J K}$ and $\tilde{C}_{I J K}$ are related by (2.33). The change $C_{I J K} \rightarrow \tilde{C}_{I J K}$ can be viewed as a threshold effect resulting from integrating in the extra hypermultiplets at $h^{\star}>0$, continuing to $h^{\star}<0$, and then integrating them out again.

For a pair of $\mathrm{CY}$ threefolds $X, \tilde{X}$ related by a conifold transition, however, an analog of the relation (2.38) remains to be established as the threshold corrections arising from integrating out the massive modes on the $\tilde{X}$ branch have not been computed. In this context we point out that knowing the triple intersection numbers of $X$ and $\tilde{X}$ explicitly and applying (2.37) to find the In-picture $\hat{C}_{I J K}$ gives a prediction for these corrections.

Let us also remark that it does not make sense to use the extended action $\hat{S}$ far away from the transition locus, where the extra states have a considerable mass, because the full M-theory contains many other massive states which are not included in $\hat{S}$. Moreover, there are additional boundaries of the Kähler cones of $X$ and $\tilde{X}$, where some other states become massless.

\footnotetext{
${ }^{14}$ Comparing to [5] the correction term differs by a factor of $\frac{1}{6}$ which is due to our different normalization of $h^{I}$.
} 


\subsection{Kasner cosmologies}

Besides constructing LEEA which explicitly include the extra light modes arising in topological phase transitions, we are also interested in the dynamics of the CY moduli and the space-time induced by the transition states. This will be our main focus in the sections $6-8$. When investigating these dynamics, we use the following Kasner ansatz for the five-dimensional space-time metric:

$$
d s_{5}^{2}=-\mathrm{e}^{2 \nu(t)} \mathrm{d} t^{2}+\mathrm{e}^{2 \alpha(t)} \mathrm{d} \vec{x}^{2}+\mathrm{e}^{2 \beta(t)} \mathrm{d} y^{2}
$$

Here $\vec{x}=\left(x^{1}, x^{2}, x^{3}\right)$ are three space-like coordinates, parametrizing the macroscopic dimensions, while $y$ is the coordinate of the fifth, extra dimension. Note that we include a non-trivial lapse function $\mathrm{e}^{\nu(t)}$ in the ansatz. This will play a crucial role in solving the Einstein equations in the Out-picture analytically. We also impose that all fields are homogeneous in the four space-like directions, i.e., they do not depend on the spatial coordinates.

Moreover, we restrict ourselves to the case where the vector fields $A_{\mu}^{I}$ can be consistently set to zero, as this will considerably simplify the later analysis. In the Out-picture the equations of motion are always solved by $A_{\mu}^{I}=0$. In the Inpicture, however, the covariant derivative in the hypermultiplet kinetic term adds the contribution

$$
j_{\mu}:=\mathrm{g} \sqrt{-g} g_{X Y}(q) \partial_{\mu} q^{X} k_{\text {gauge }}^{Y}(q),
$$

to the vector field equations of motion which does not vanish when setting $A_{\mu}^{I}=0$. For the In-picture Lagrangians constructed in the subsequent sections, this term vanishes, if the complex hypermultiplet scalar fields $q^{X}$ are restricted to be real. Therefore we will use this truncation when studying the dynamics of these theories.

Under these assumptions we get the following non-trivial equations of motion from the Lagrangian (2.1): 
Einstein equations:

$$
\begin{aligned}
3\left(\dot{\alpha}^{2}+\dot{\alpha} \dot{\beta}\right) & =T+\mathrm{g}^{2} \mathrm{e}^{2 \nu} \mathbb{V}, \\
2 \ddot{\alpha}+\ddot{\beta}+2 \dot{\alpha} \dot{\beta}+3 \dot{\alpha}^{2}+\dot{\beta}^{2}-2 \dot{\nu} \dot{\alpha}-\dot{\nu} \dot{\beta} & =-T+\mathrm{g}^{2} \mathrm{e}^{2 \nu} \mathbb{V}, \\
3\left(\ddot{\alpha}+2 \dot{\alpha}^{2}-\dot{\nu} \dot{\alpha}\right) & =-T+\mathrm{g}^{2} \mathrm{e}^{2 \nu} \mathbb{V},
\end{aligned}
$$

vector multiplet sector:

$$
\ddot{\phi}^{x}+\gamma_{y z}^{x} \dot{\phi}^{y} \dot{\phi}^{z}+(3 \dot{\alpha}+\dot{\beta}-\dot{\nu}) \dot{\phi}^{x}+\mathrm{e}^{2 \nu} \mathrm{g}^{2} g^{x y} \frac{\partial \mathbb{V}}{\partial \phi^{y}}=0
$$

hypermultiplet sector:

$$
\ddot{q}^{X}+\Gamma_{Y Z}^{X} \dot{q}^{Y} \dot{q}^{Z}+(3 \dot{\alpha}+\dot{\beta}-\dot{\nu}) \dot{q}^{X}+\mathrm{e}^{2 \nu} \mathrm{g}^{2} g^{X Y} \frac{\partial \mathbb{V}}{\partial q^{Y}}=0
$$

Here the "overdot" indicates a derivative with respect to the time coordinate $t$, i.e., $\dot{q}^{X}:=\frac{\partial}{\partial t} q^{X}$, etc. We also introduced the kinetic energy $T$ as

$$
T:=\frac{1}{2} g_{X Y} \dot{q}^{X} \dot{q}^{Y}+\frac{1}{2} g_{x y} \dot{\phi}^{x} \dot{\phi}^{y}
$$

which is positive semi-definite. The $\gamma_{y z}^{x}$ and $\Gamma_{Y Z}^{X}$ denote the Christoffel symbols of the vector and hypermultiplet scalar metrics $g_{x y}$ and $g_{X Y}$, respectively. To obtain the equations of motion valid in the Out- or In-picture, we make the appropriate substitutions for the potential and the scalar field metrics. When studying numerical solutions, g will be fixed to the value determined by M-theory, eq. (4.16). 


\section{The hypermultiplet target manifolds}

In subsection 2.3 we determined the exact vector multiplet sector of the In-picture LEEA including the transition states originating from a flop or conifold transition. Our next task is the construction of a family of hypermultiplet target manifolds which can be used to describe the additional charged hypermultiplets arising in these transitions. We will not attempt to derive these manifolds directly from Mtheory, but use the Wolf spaces (1.1). In order to find the explicit LEEA we need to know the metrics, the Killing vectors, and the moment maps of these spaces explicitly. As already mentioned, the Wolf spaces also happen to be Kähler, so that one can deduce these quantities from the corresponding Kähler potential. However, the structure relevant for the gauging of isometries is the quaternionic structure, as the scalar potential depends on the moment maps of the Killing vectors, which form a triplet under the $S U(2)$ related to the quaternionic structure. Therefore we will construct these objects from their counterparts on the associated hyper-Kähler cone using the superconformal quotient construction [40,41, 42]. This method can be applied to any quaternion-Kähler space.

The construction of the Wolf spaces (1.1) has been described in [40] but explicit formulae for the metric have only been given for $X(1)$. The general form of the Killing vectors of $X(N+1)$ has been obtained in [41]. In this section we summarize the explicit formulae for the metrics, Killing vectors, and moment maps of all these spaces obtained in the appendix D. As it will turn out in the next sections, the resulting parametrization is extremely useful for including the transition states in the LEEA.

Before considering the particular family (1.1) of quaternion-Kähler spaces, let us briefly explain the underlying method. From the physical point of view the basic idea is to construct theories with Poincaré supersymmetry as gauge-fixed versions of superconformal theories. In the case at hand one starts with a theory of $n=$ 
$N+2$ hypermultiplets ${ }^{15}$ invariant under rigid superconformal transformations. The corresponding hypermultiplet manifold $\mathcal{M}_{\mathrm{HM}}^{\mathrm{SC}}$ is a hyper-Kähler cone, i.e., it is hyperKähler and, in addition, possesses a homothetic Killing vector $\chi^{a}$ satisfying $D_{a} \chi^{b}=$ $\delta_{a}^{b}$. This implies that the hyper-Kähler metric $g_{a b}$ of $\mathcal{M}_{\mathrm{HM}}^{\mathrm{SC}}$ has a hyper-Kähler potential $\chi$, with $\chi_{a}=D_{a} \chi$ and $g_{a b}=D_{a} D_{b} \chi$. Moreover, $\mathcal{M}_{\mathrm{HM}}^{\mathrm{SC}}$ is a cone over a so-called tri-Sasakian manifold with radial coordinate $r=\sqrt{2 \chi}$. Superconformal invariance also implies that by multiplying the homothety $\chi^{a}$ with the $S U(2)$ triplet of complex structures $\vec{J}=\left[J^{+}, J^{-}, J^{3}\right]$ of $\mathcal{M}_{\mathrm{HM}}^{\mathrm{SC}}$ one obtains an $S U(2)$ triplet of Killing vectors,

$$
\vec{k}^{a}=\vec{J}_{b}^{a} \chi^{b}
$$

Using the superconformal calculus, the rigid superconformal theory can be coupled to conformal supergravity and thus be promoted to a locally superconformal theory. This theory is gauge-equivalent to a theory of $n-1$ hypermultiplets coupled to Poincaré supergravity. In this reinterpretation one of the hypermultiplets becomes dependent on the other fields and acts as a compensator. Geometrically this gauging corresponds to performing a superconformal quotient of $\mathcal{M}_{\mathrm{HM}}^{\mathrm{SC}}$ with respect to the four conformal Killing vector fields $\chi^{a}, \vec{k}^{a}$. The resulting hypermultiplet manifold $\mathcal{M}_{\mathrm{HM}}$ of the Poincaré supergravity theory is quaternion-Kähler. In fact every quaternion-Kähler manifold can be obtained by this construction from its associated hyper-Kähler cone [39].

The construction of the Wolf spaces $X(n-1)$ which have $\operatorname{dimension} \operatorname{dim}_{\mathbb{R}}(X(n-$ $1))=4(n-1)$ proceeds in several steps. First one needs to obtain the hyperKähler cone $\mathcal{H}^{(2 n)}$ associated with the space $X(n-1)$. In [40] this cone has been constructed as the hyper-Kähler quotient [69] of flat $\mathbb{C}^{(2 n+2)}$ with respect to a particular tri-holomorphic $U(1)$ isometry. Then the superconformal quotient is taken in two steps. First one quotients $\mathcal{H}^{(2 n)}$ by the homothetic Killing vector $\chi^{a}$ and the Killing vector $k^{3}=\left[k^{3 a}\right]$ corresponding to the Cartan direction of the $S U(2)$

\footnotetext{
${ }^{15}$ For notational convenience we have set $N+1=n-1$.
} 
isometry group. This quotient is a standard Kähler quotient [70]. The resulting space is the twistor space $\mathcal{Z}^{(2 n-2)}$ over $X(n-1)$. In the second step one quotients $\mathcal{Z}^{(2 n-2)}$ by the remaining Killing vectors $k^{+}$and $k^{-}$. The isometry (3.1), however, is only holomorphic and not tri-holomorphic. This implies that at the level of the twistor space, $k^{+}$and $k^{-}$are isometries up to $S U(2)$ rotations only. In order to obtain well defined quantities on the quaternion-Kähler manifold one has to include a compensating $S U(2)$ transformation.

\subsection{Results for the metric and isometries of $X(n-1)$}

The actual derivation of the explicit formulae for the metric, Killing vectors and moment maps of the Wolf spaces $X(n-1)$ is, however, rather technical and will not be required in the subsequent sections of this thesis. Therefore this subsection only gives a summary of our results while the calculation is contained in appendix D.

We start by introducing complex coordinates $v^{i}, u_{i}, i=1, \ldots, n-1$ with respect to the almost complex structure $J^{3}$, corresponding to the $n-1$ hypermultiplets of $X(n-1)$. Arranging our indices as $X, Y=\left\{v^{i}, \bar{v}^{\bar{\imath}}, u_{i}, \bar{u}_{\bar{\imath}}\right\}$ the components of the metric $G_{\alpha \bar{\beta}}$ on $X(n-1)$ can be read off from the matrix

$$
G_{X Y}=\left[\begin{array}{cccc}
0 & G_{v \bar{v}} & 0 & G_{v \bar{u}} \\
G_{\bar{v} v} & 0 & G_{\bar{v} u} & 0 \\
0 & G_{u \bar{v}} & 0 & G_{u \bar{u}} \\
G_{\bar{u} v} & 0 & G_{\bar{u} u} & 0
\end{array}\right]
$$


The entries of this matrix are given by

$$
\begin{aligned}
G_{u_{i} \bar{u}_{\bar{\jmath}}}= & \frac{1}{2 \phi_{-}}\left(\eta^{i \bar{\jmath}}+\bar{v}^{\bar{\jmath}} v^{i}\right)-\frac{1}{2 \phi_{-}^{2}}\left(\eta^{\bar{l} l} u_{l}+\bar{v}^{\bar{\jmath}}\left(v^{l} u_{l}\right)\right)\left(\eta^{i \bar{l}} \bar{u}_{\bar{l}}+v^{i}\left(\bar{v}^{\bar{l}} \bar{u}_{\bar{l}}\right)\right) \\
G_{\bar{v}^{\bar{\imath}} u_{j}}= & \frac{1}{2 \phi_{-}^{2}}\left(\bar{u}_{\bar{\imath}} v^{j}\left(1+\eta^{k \bar{l}} u_{k} \bar{u}_{\bar{l}}\right)-\bar{u}_{\bar{\imath}} \eta^{j \bar{l}} \bar{u}_{\bar{l}}\left(v^{l} u_{l}\right)\right) \\
G_{v^{i} \bar{v}^{\bar{\jmath}}}= & \frac{1}{2 \phi_{+}} \eta_{i \bar{\jmath}}-\frac{1}{2 \phi_{+}^{2}}\left(\eta_{i \bar{l}} \bar{v}^{\bar{l}}\right)\left(\eta_{\bar{\jmath} l} v^{l}\right)-\frac{1}{\phi_{+} \phi_{-}} u_{i} \bar{u}_{\bar{\jmath}} \\
& +\frac{1}{2 \phi_{-}} u_{i} \bar{u}_{\bar{\jmath}}-\frac{1}{2 \phi_{-}^{2}} u_{i} \bar{u}_{\bar{\jmath}}\left(v^{l} u_{l}\right)\left(\bar{v}^{\bar{l}} \bar{u}_{\bar{l}}\right)
\end{aligned}
$$

Here we introduced the $(n-1) \times(n-1)$ matrix $\eta_{i \bar{\jmath}}:=\operatorname{diag}[-1, \ldots-1]$ and its inverse $\eta^{i \bar{\jmath}}$ while $\phi_{+}$and $\phi_{-}$are given by

$$
\phi_{+}:=1+\eta_{i \bar{\jmath}} v^{i} \bar{v}^{\bar{\jmath}}, \quad \phi_{-}:=1+\eta^{i \bar{\jmath}} u_{i} \bar{u}_{\bar{\jmath}}+\left(v^{i} u_{i}\right)\left(\bar{v}^{\bar{\imath}} \bar{u}_{\bar{\imath}}\right)
$$

The other non-vanishing entries of the matrix (3.2) can be obtained from the condition that $G_{X Y}$ is hermitian with respect to $J^{3}$. These metrics are, however, not Kähler as the holomorphic assignment in (3.3) is adapted to the quaternionic structure, which cannot be used to define a Kähler potential. However, there must be a non-holomorphic coordinate transformation which brings the metric given above into its Kähler form [37].

The universal hypermultiplet $X(1)$ is obtained by setting $n=2$. In this case the index $i$ has only a single value and may be omitted. Setting $\eta_{i \bar{\jmath}}=\eta_{1 \overline{1}}=-1$ the general metric (3.3) becomes

$$
\begin{aligned}
G_{u \bar{u}} & =-\frac{1}{2 \phi_{-}^{2}}(1-v \bar{v}), \\
G_{u \bar{v}} & =\frac{1}{2 \phi_{-}^{2}} \bar{u} v, \\
G_{v \bar{v}} & =-\frac{1}{2 \phi_{+}^{2} \phi_{-}^{2}}\left(1-u \bar{u}(1-v \bar{v})^{2}\right),
\end{aligned}
$$

which is the metric for the universal hypermultiplet derived in [40]. 
The Killing vectors of the general metric (3.2) are conveniently obtained in terms of the generators $\left[t_{\alpha}\right]_{I}^{J}$ of the Lie algebra $s u(n-1,2)$. Dropping the index $\alpha$ enumerating the $(n+1)^{2}-1$ generators of $s u(n-1,2)$ and splitting the matrix index $I=i, n, n+1$, their components read

$$
\begin{gathered}
\hat{k}^{v^{i}}=i t^{i}{ }_{j} v^{j}+i t_{n+1}^{i}-i v^{i} t^{n+1}{ }_{j} v^{j}-i v^{i} t_{n+1}^{n+1}-\frac{k^{\zeta}}{2}\left(\eta^{i \bar{\jmath}} \bar{u}_{\bar{\jmath}}+v^{i} \bar{v}^{\bar{k}} \bar{u}_{\bar{k}}\right) \\
\hat{k}^{u_{i}}=i u_{i}\left(t^{n+1} v^{j}+t_{n+1}^{n+1}\right)-i t^{j}{ }_{i} u_{j}-i t_{i}^{n}+i t_{i}^{n+1}\left(v^{j} u_{j}\right) \\
-2 u_{i} k_{z}+\frac{\phi_{-}}{2 \phi_{+}} k^{\zeta} \eta_{i \bar{\jmath}} \bar{v}^{\bar{\jmath}}
\end{gathered}
$$

with $k^{\zeta}$ and $k_{z}$ given by

$$
\begin{aligned}
k^{\zeta} & =2 i\left(t_{i}^{n} v^{i}+t_{n+1}^{n}\right) \quad \text { and } \\
k_{z} & =\frac{i}{2}\left(t_{i}^{n+1} v^{i}+t_{n+1}^{n+1}-t_{n}^{i} u_{i}-t_{n}^{n}+t_{n}^{n+1} v^{i} u_{i}\right) .
\end{aligned}
$$

The components of the Killing vectors with respect to $\partial_{\bar{v} \bar{\imath}}$ and $\partial_{\bar{u}_{\bar{\imath}}}$ are obtained from eq. (3.6) by complex conjugation.

The $S U(2)$ triplet of moment maps $\hat{\mu}^{r}, r=+,-, 3$, associated with these Killing vectors is also obtained from its counterpart on the hyper-Kähler cone,

$$
\begin{aligned}
& \hat{\mu}^{3}=-\frac{1}{2 \phi_{+}}\left\{\bar{v}^{\bar{\imath}} \eta_{\bar{\imath} j} t_{k}^{j} v^{k}+t_{i}^{n+1} v^{i}+\bar{v}^{\bar{\jmath}} \eta_{\bar{\jmath} i} t_{n+1}^{i}+t_{n+1}^{n+1}\right\} \\
&+\frac{1}{2 \phi_{-}}\left\{u_{i} t^{i}{ }_{j} \eta^{j \bar{k}} \bar{u}_{\bar{k}}+u_{i} t_{n}^{i}+t^{n}{ }_{i} \eta^{i \bar{\jmath}} \bar{u}_{\bar{\jmath}}+t^{n}{ }_{n}-\left(u_{i} t^{i}{ }_{n+1}+t^{n}{ }_{n+1}\right)\left(\bar{u}_{\bar{\imath}} \bar{v}^{\bar{\imath}}\right)\right. \\
&\left.-\left(t^{n+1}{ }_{i} \eta^{i \bar{\jmath}} \bar{u}_{\bar{\jmath}}+t^{n+1}\right)\left(u_{k} v^{k}\right)+t^{n+1}{ }_{n+1}\left(u_{i} v^{i}\right)\left(\bar{u}_{\bar{\jmath}} \bar{v}^{\bar{\jmath}}\right)\right\} \\
& \hat{\mu}^{+}=-\frac{i}{2 \phi_{+}^{1 / 2} \phi_{-}^{1 / 2}}\left\{u_{i} t^{i}{ }_{j} v^{j}+t^{n}{ }_{j} v^{j}-t^{n+1}{ }_{i} v^{i}\left(u_{j} v^{j}\right)+u_{i} t^{i}{ }_{n+1}\right. \\
&\left.+t_{n+1}^{n}-t_{n+1}^{n+1}\left(u_{i} v^{i}\right)\right\}
\end{aligned}
$$

with $\hat{\mu}^{-}$and $\hat{\mu}^{+}$related by complex conjugation. This equation completes our summary of the results obtained in appendix D. We now have all the ingredients for modelling the hypermultiplet sector of our In-picture LEEA. 


\subsection{Examples of isometries on $X(2)$}

Before we embark on this construction, let us illustrate how the general formulae (3.6) and (3.8) may be used. For this purpose we anticipate some results obtained in the next sections and calculate the Killing vectors and moment maps for the flop and conifold LEEA with hypermultiplet sector based on $X(2)$. It turns out that the corresponding isometries are generated by

$$
t_{\text {Flop }}=\frac{1}{4} \operatorname{diag}[1,-3,1,1]
$$

and

$$
t_{\mathrm{Cf}}=\operatorname{diag}[1,-1,0,0],
$$

respectively. The relative normalization of these generators is chosen such that they give rise to the same mass on the Coulomb branch of the transition. Substituting these matrices into eq. (3.6), we find the corresponding Killing vectors,

$$
\begin{aligned}
k_{\text {Flop }} & =-i\left[0, v^{2}, 0,-\bar{v}^{2}, 0,-u_{2}, 0, \bar{u}_{2}\right]^{\mathrm{T}}, \\
k_{\mathrm{Cf}} & =i\left[v^{1},-v^{2},-\bar{v}^{1}, \bar{v}^{2},-u_{1}, u_{2}, \bar{u}_{1},-\bar{u}_{2}\right]^{\mathrm{T}},
\end{aligned}
$$

which are given with respect to the basis

$$
\left\{\partial_{v^{1}}, \partial_{v^{2}}, \partial_{\bar{v}^{1}}, \partial_{\bar{v}^{2}}, \partial_{u_{1}}, \partial_{u_{2}}, \partial_{\bar{u}_{1}}, \partial_{\bar{u}_{2}}\right\}
$$

When gauging these isometries we also need the triplet of moment maps associ- 
ated with these Killing vectors. They are found by evaluating eq. (3.8):

$$
\begin{aligned}
\hat{\mu}_{\text {Flop }}^{r}= & {\left[\begin{array}{c}
\frac{i}{2 \phi_{+}^{1 / 2} \phi_{-}^{1 / 2}}\left(v^{2} u_{2}-\bar{v}^{2} \bar{u}_{2}\right) \\
\frac{1}{2 \phi_{+}^{1 / 2} \phi_{-}^{1 / 2}}\left(v^{2} u_{2}+\bar{v}^{2} \bar{u}_{2}\right) \\
\frac{1}{2 \phi_{-}}\left(\bar{u}_{2} u_{2}\right)-\frac{1}{2 \phi_{+}}\left(\bar{v}^{2} v^{2}\right)
\end{array}\right], } \\
\hat{\mu}_{\mathrm{Cf}}^{r}= & {\left[\begin{array}{c}
-\frac{i}{2 \phi_{+}^{1 / 2} \phi_{-}^{1 / 2}}\left(v^{1} u_{1}-v^{2} u_{2}-\bar{v}^{1} \bar{u}_{1}+\bar{v}^{2} \bar{u}_{2}\right) \\
-\frac{1}{2 \phi_{+}^{1 / 2} \phi_{-}^{1 / 2}}\left(v^{1} u_{1}-v^{2} u_{2}+\bar{v}^{1} \bar{u}_{1}-\bar{v}^{2} \bar{u}_{2}\right) \\
-\frac{1}{2 \phi_{-}}\left(u_{1} \bar{u}_{1}-u_{2} \bar{u}_{2}\right)+\frac{1}{2 \phi_{+}}\left(v^{1} \bar{v}^{1}-v^{2} \bar{v}^{2}\right)
\end{array}\right] . }
\end{aligned}
$$

Here the components of the moment maps $\left\{\hat{\mu}^{1}, \hat{\mu}^{2}, \hat{\mu}^{3}\right\}$ are adapted to the basis associated with the complex structures given in (D.2). Their relation to $\hat{\mu}^{+}$and $\hat{\mu}^{-}$ is given by

$$
\hat{\mu}^{1}=\hat{\mu}^{+}+\hat{\mu}^{-}, \quad \hat{\mu}^{2}=-i\left(\hat{\mu}^{+}-\hat{\mu}^{-}\right), \quad \hat{\mu}^{3}=\hat{\mu}^{3} .
$$

This completes our excursion on isometries in the two hypermultiplet case.

\subsection{The relation to the supergravity conventions}

In order to use the results obtained in this section to construct a supergravity Lagrangian based on eq. (2.1) we need to rewrite them in the conventions used in section 2.1. Comparing the metric for the universal hypermultiplet given in [59] and [40] we find that the components of the metric $G_{X Y}$ given in (3.2) and the metric $g_{X Y}$ in the Lagrangian (2.1) are related by

$$
g_{X Y}(q)=-G_{X Y}(q)
$$

Looking at the definitions of the moment map (2.15) and the one given in [41], we further find that these differ by a factor of one half,

$$
P_{I}^{r}(q)=\frac{1}{2} \hat{\mu}^{r}(q)
$$


When studying cosmological solutions it is further interesting to identify a subspace of the hypermultiplet target manifold $X(n-1)$ with the universal hypermultiplet $X(1)$ which, for CY compactifications, contains the CY volume $V$ and three real scalars $\sigma, \theta, \tau$ stemming from the reduction of the three-from. To this end we observe that restricting the metric on $X(n-1)$ to the subspace spanned by $v^{1}, u_{1}$ with all other coordinates set to zero gives rise to the metric (3.5) for $X(1)$. By combining the coordinate transformations given in $[40,59]$ it is then straightforward to relate the parametrization of the universal hypermultiplet $X(1)$ given by $g_{X Y}(q)$ obtained from (3.5) to the one derived from CY-compactifications of M-theory [71],

$$
d s^{2}=\frac{1}{4 V^{2}} \mathrm{~d} V^{2}+\frac{1}{4 V^{2}}(\mathrm{~d} \sigma+2 \theta \mathrm{d} \tau-2 \tau \mathrm{d} \theta)^{2}+\frac{1}{V}\left(\mathrm{~d} \theta^{2}+\mathrm{d} \tau^{2}\right)
$$

Explicitly, we find

$$
\begin{aligned}
V & =\frac{1}{L}\left(1-u \bar{u}(1-v \bar{v})^{2}-v \bar{v}\right) \\
\sigma & =\frac{i}{L}(u-\bar{u})(1-v \bar{v}) \\
\theta & =\frac{1}{2 L}(\bar{v}(1+\bar{u}(1-v \bar{v}))+v(1+u(1-v \bar{v}))) \\
\tau & =\frac{i}{2 L}(\bar{v}(1+\bar{u}(1-v \bar{v}))-v(1+u(1-v \bar{v})))
\end{aligned}
$$

where $L$ is given by

$$
L:=(1+u(1-v \bar{v}))(1+\bar{u}(1-v \bar{v})) .
$$

Therefore we recover the eleven-dimensional meaning of the coordinates $v, u$ parametrizing $X(1)$ in (3.5) and in particular their relation to the CY volume $V$. We note that taking $v=\bar{v}=0$ and $u=\bar{u}$ corresponds to setting $\sigma=\theta=\tau=0$ and truncating the universal hypermultiplet to the volume scalar $V$. 


\section{The flop transition}

We now have all the ingredients to write down the explicit In-picture LEEA for general flop transitions including the charged extra states which become massless at the transition locus. To illustrate the key features of the construction, we first consider an example based on the $\mathbb{F}_{1}$-model reviewed in appendix $\mathrm{C}$ before turning to the generic transition. In both cases we proceed by first deriving the Lagrangian and then showing that the scalar masses obey the conditions arising from the microscopic picture.

\subsection{The Lagrangian of the $\mathbb{F}_{1}$-model}

\section{Gauging the general supergravity action}

According to the microscopic description of the flop transition occurring in the $\mathbb{F}_{1}$ model the In-picture Lagrangian should contain one neutral and one charged hypermultiplet. These play the roles of the universal hypermultiplet and of the transition states, respectively. ${ }^{16}$ The latter are charged with respect to the vector field $A_{\mu}^{\star}$ whose associated cycle collapses at the flop. In our particular model this implies that the transition states are charged with respect to the vector field associated with the scalar field combination $(U-W)$, as this is the modulus that vanishes at the transition locus. Taking the hypermultiplet scalar manifold $\mathcal{M}_{\mathrm{HM}}$ to be $X(2)$ with complex coordinates $v^{1}, v^{2}, u_{1}, u_{2}$, we choose the universal hypermultiplet as being represented by $v^{1}, u_{1}$, while the transition states are given by $v^{2}, u_{2}$.

Our first task is to identify the proper gauging in the hypermultiplet sector. Here we need a Killing vector which encodes the $U(1)$ charges of the second hypermultiplet. This implies that the correct Killing vector should be independent

\footnotetext{
${ }^{16} \mathrm{As}$ explained in the introduction, our model only includes the universal hypermultiplet and the transition states. The additional neutral hypermultiplets arising in the CY compactification are frozen and will not be included in the following analysis.
} 
of $v^{1}, u_{1}$, should not act on the universal hypermultiplet, and that its components with respect to $\partial_{v^{2}}$ and $\partial_{u_{2}}$ are of the form $-i v^{2}$ and $i u_{2}$, respectively. ${ }^{17}$ Inspecting the expression for a general Killing vector on $X(2)(3.6)$, it turns out that these specifications determine the Killing vector uniquely. ${ }^{18}$ Anticipating this result, this Killing vector, $k_{\text {Flop }}$, and its associated triplet of moment maps $\hat{\mu}_{\text {Flop }}^{r}$ have been given in eqs. (3.11) and (3.14), respectively.

In the next step we perform the gauging of (2.1) with respect to this isometry. In order to compare our five-dimensional supergravity action with eleven-dimensional M-theory data, it is natural to use the embedding coordinates $h^{I},($ C.1), as these are the coordinates which are related to the volumes of the CY cycles. However, for the $\mathbb{F}_{1}$-model it is more convenient to work with the variables $T, U, W$ given in (C.3). Further, it is useful to label the vector fields $A_{\mu}^{I}$ by their corresponding scalar field:

$$
\left\{A_{\mu}^{I}, \quad I=0,1,2\right\} \quad \longrightarrow \quad\left\{A_{\mu}^{T}, A_{\mu}^{U}, A_{\mu}^{W}\right\}
$$

Next we consider the scalar kinetic terms of (2.1). Since we do not gauge any isometries of the vector multiplet scalar manifold, the corresponding gauge covariant derivative becomes a partial derivative,

$$
\mathcal{D}_{\mu} \phi^{x}=\partial_{\mu} \phi^{x} \quad \Leftrightarrow \quad K_{I}^{x}(\phi) \quad \text { trivial }
$$

In the hypermultiplet sector the microscopic picture fixes the $U(1)$ gauge connection of the isometry (3.11) to be $A_{\mu}^{U}-A_{\mu}^{W}$. To implement this requirement we set

$$
K_{T}^{X}(q)=0, \quad K_{U}^{X}(q)=k_{\text {Flop }}^{X}(q), \quad K_{W}^{X}(q)=-k_{\text {Flop }}^{X}(q) .
$$

\footnotetext{
${ }^{17}$ Here and in the following we use conventions in which the charged hypermultiplet fields $u_{i}$ carry $U(1)$ charge $q=+1$ while their CPT conjugate partners $v^{i}$ have charge $q=-1$.

${ }^{18}$ Any rescaling of the Killing vector can be absorbed by a rescaling of the gauge coupling $g$. We will fix the normalization of the Killing vector and show later that $g$ is uniquely determined by microscopic M-theory physics.
} 
The covariant derivative for the hypermultiplet scalars then becomes

$$
\mathcal{D}_{\mu} q^{X}=\partial_{\mu} q^{X}+\mathrm{g}\left(A_{\mu}^{U}-A_{\mu}^{W}\right) k_{\mathrm{Flop}}^{X}(q)
$$

This expression shows that the universal hypermultiplet parametrized by $v^{1}, u_{1}$ is neutral, while the transition states $v^{2}, u_{2}$ carry $U(1)$ charges $q=-1$ and $q=+1$ with respect to the gauge connection, respectively.

Next we turn to the scalar potential (2.20) where we take the independent vector multiplet scalar fields as $\phi^{x}=U, W$, while $T(U, W)$ is given in eq. (C.9). Including the rescaling (3.18) the $P_{I}^{r}$ are given by

$$
P_{T}^{r}(q)=0, \quad P_{U}^{r}(q)=\frac{1}{2} \hat{\mu}_{\mathrm{Flop}}^{r}(q), \quad P_{W}^{r}(q)=-\frac{1}{2} \hat{\mu}_{\mathrm{Flop}}^{r}(q)
$$

Correspondingly, $P^{r}$ is obtained as

$$
P^{r}=\frac{1}{2} h^{1} \hat{\mu}_{\text {Flop }}^{r}=\frac{1}{2} 6^{-1 / 3}(U-W) \hat{\mu}_{\text {Flop }}^{r},
$$

where we used (C.3) in the second step.

In order to construct the scalar potential, we work out the superpotential (2.21). For the $P^{r}$ above this is given by

$$
\mathcal{W}=6^{-5 / 6}\left(\frac{1}{2 \phi_{-}}\left(\bar{u}_{2} u_{2}\right)+\frac{1}{2 \phi_{+}}\left(\bar{v}^{2} v^{2}\right)\right)(U-W)
$$

It is now straightforward to check that the $Q^{r}$ defined in $(2.22)$ is independent of the vector multiplet scalars,

$$
Q^{r}=\sqrt{\frac{2}{3}} \frac{P^{r}}{\mathcal{W}}=\frac{\hat{\mu}_{\mathrm{Flop}}^{r}(q)}{\frac{1}{2 \phi_{-}}\left(\bar{u}_{2} u_{2}\right)+\frac{1}{2 \phi_{+}}\left(\bar{v}^{2} v^{2}\right)} .
$$

Hence the condition $\partial_{x} Q^{r}=0$ is trivially satisfied. This implies that the scalar 
potential can be written as

$$
\mathbb{V}(\phi, q)=-6 \mathcal{W}^{2}+\frac{9}{2} g^{\Lambda \Sigma} \partial_{\Lambda} \mathcal{W} \partial_{\Sigma} \mathcal{W}
$$

Here $g^{\Lambda \Sigma}$ is defined in (2.24) with $g^{X Y}(q)$ being (minus) the inverse metric on $X(2)$ and $g^{x y}(\phi)$ the inverse of the In-picture vector multiplet scalar metric (C.10). The coordinates of the scalar manifold $\mathcal{M}_{\mathrm{HM}} \otimes \mathcal{M}_{\mathrm{VM}}$ are taken to be

$$
\phi^{\Lambda}=\left\{v^{1}, v^{2}, \bar{v}^{1}, \bar{v}^{2}, u_{1}, u_{2}, \bar{u}_{1}, \bar{u}_{2}, U, W\right\}
$$

Alternatively, we can compute the scalar potential by substituting the quantities $K^{X}, P^{r}, P_{x}^{r}$, and the inverse vector and hypermultiplet scalar metrics directly into the scalar potential (2.20). By explicit computation one finds that the resulting expressions agree. Since the equality of (2.23) and (2.20) requires some non-trivial identities of quaternion-Kähler geometry, this result provides a non-trivial check for our derivation.

\section{Vacua and mass matrix}

After constructing the In-picture Lagrangian for our flop model, let us investigate its vacuum structure and calculate the corresponding mass matrix. From the microscopic analysis we know that the masses of the transition states must be proportional to $|U-W|$ while all other fields must be massless.

The supersymmetric vacua of $\mathbb{V}$ can then be found via the algebraic eqs. (2.25). For the specific gauging $(4.3),(4.5)$ these equations are solved by $v^{2}=u_{2}=0$, with the value of the other fields $v^{1}, u_{1}, U, W$ undetermined. This implies that we have an entire manifold $\mathcal{M}_{\text {Coul }}$ of critical points, corresponding to a Coulomb branch parametrized by the vacuum expectation values of the universal hypermultiplet and 
the vector multiplet scalars:

$$
\left.\partial_{\Lambda} \mathbb{V}\right|_{\mathcal{M}_{\text {Coul }}}=0, \quad \mathcal{M}_{\text {Coul }}=\left\{\begin{array}{l}
v^{2}=u_{2}=0 \\
v^{1}, u_{1}, U, W \text { not fixed by eq. }(2.25) .
\end{array}\right.
$$

When investigating the possibility that $\mathbb{V}$ has additional (possibly non-supersymmetric) vacua, it first seems like there are some other critical points. A thorough investigation of these points, however, establishes that they are outside the admissible coordinate region of the scalar manifolds, i.e., at these points the scalar metric $g_{\Sigma \Lambda}$ is no longer positive definite.

To determine the type of vacuum corresponding to this set of critical points, we substitute the condition for a critical point into the potential (4.9). Since both $\mathcal{W}$ and $\partial_{\Lambda} \mathcal{W}$ vanish at $v^{2}=u_{2}=0$, we find

$$
\left.\mathbb{V}(\phi, q)\right|_{\mathcal{M}_{\text {Coul }}}=0
$$

Hence the manifold $\mathcal{M}_{\text {Coul }}$ corresponds to a set of Minkowski vacua with vanishing cosmological constant.

We now calculate the masses of the scalars in our model. These are given by the eigenvalues of the mass matrix

$$
\mathcal{M}_{\Sigma}^{\Lambda}=\left.g^{2} g^{\Lambda \Xi} \frac{\partial}{\partial \phi^{\Xi}} \frac{\partial}{\partial \phi^{\Sigma}} \mathbb{V}(\phi, q)\right|_{\mathcal{M}_{\text {Coul }}}
$$

where $g^{\Lambda \Sigma}$ is given in eq. (2.24). Evaluating this expression for the potential (4.9) we find

$$
\mathcal{M}_{\Sigma}^{\Lambda}=\left(m_{\text {Flop }}\right)^{2} \operatorname{diag}[0,1,0,1,0,1,0,1,0,0]
$$

given with respect to the basis (4.10). This result shows that the universal hypermultiplet $v^{1}, u_{1}$ and the vector multiplet scalars $U, W$ are massless and parametrize the flat directions of the potential. The masses of the transition states $v^{2}, u_{2}$ are 
given by

$$
\left(m_{\text {Flop }}\right)^{2}=\frac{3}{2} \mathrm{~g}^{2} 6^{-2 / 3}(U-W)^{2}=\frac{3}{2} \mathrm{~g}^{2}\left(h^{1}\right)^{2}
$$

In terms of the microscopic picture $\left|h^{1}\right|=6^{-1 / 3}|U-W|$ corresponds to the volume of the shrinking cycle. This implies that (4.15) has precisely the structure expected from the eleven-dimensional point of view. By comparing with (2.36) and using (2.31) together with the value $T_{(2)}=\left(\frac{8 \pi}{\kappa_{(11)}^{2}}\right)^{1 / 3}$ of the M2-brane tension [13], we find that $\mathrm{g}$ is fixed by M-theory,

$$
\mathrm{g}=\sqrt{\frac{2}{3}}(48 \pi)^{1 / 3}
$$

Thus the In-picture LEEA is completely fixed once we choose the hypermultiplet manifold to be $X(2)$.

\subsection{The Lagrangian for general flop transitions}

After working out the In-picture Lagrangian for an explicit model containing a single charged hypermultiplet, we will now generalize this setup to a generic flop where $N$ hypermultiplets become massless at the transition locus. Our construction does not depend on the details of the vector multiplet scalar manifolds connected by the flop and can easily be adjusted to any specific transition. The relation between Out-picture and In-picture is given by the orbit sum rule (2.38). After fixing the hypermultiplet scalar manifold to be $X(N+1)$, we find that the resulting hypermultiplet sector of these In-picture Lagrangians is still uniquely determined by the microscopic theory.

\section{Constructing the action}

For a generic flop transition the homology class $C^{\star}=q_{I} C^{I}$ of the contracting cycle contains $N$ isolated holomorphic curves. In this case the transition states are given 
by $N$ hypermultiplets, which carry $U(1)$ charge $q= \pm 1$ with respect to the vector field $A_{\mu}^{\star}=q_{I} A_{\mu}^{I}$ associated to $C^{\star} \cdot{ }^{19}$ Generalizing our construction from the previous section, we take the hypermultiplet scalar manifold to be $X(N+1)$, which will contain the universal hypermultiplet $v^{1}, u_{1}$ and $N$ charged hypermultiplets $v^{\alpha}, u_{\alpha}$. Here the index $\alpha=2, \ldots, N+1$ enumerates the charged hypermultiplets which correspond to the transition states. We further use $h^{\star}=q_{I} h^{I}$ to denote the volume of the shrinking cycle $C^{\star}$.

The microscopic theory imposes that the transition states $v^{\alpha}, u_{\alpha}$ carry $U(1)$ charge $q=-1$ and $q=+1$ with respect to $A_{\mu}^{\star}$, while the universal hypermultiplet remains neutral. This condition requires the existence of a holomorphic Killing vector of the form:

$$
k_{\text {gauge }}^{v^{i}}=-i\left[0, v^{2}, \ldots, v^{N+1}\right]^{\mathrm{T}}, \quad k_{\text {gauge }}^{u_{i}}=i\left[0, u_{2}, \ldots, u_{N+1}\right]^{\mathrm{T}} .
$$

Since this Killing vector is holomorphic, its components $k_{\text {gauge }}^{\bar{v}^{i}}$ and $k_{\text {gauge }}^{\bar{u}_{i}}$ can be obtained from $k_{\text {gauge }}^{v^{i}}$ and $k_{\text {gauge }}^{u_{i}}$ by complex conjugation. The sign conventions and overall scale in (4.17) are chosen such that for $N=1$ we reproduce the results of the previous section.

The first step is to check whether there exists a generator $t$ which gives rise to this Killing vector. Restricting the general expression for a Killing vector on $X(N+1)$ to the terms linear in the hypermultiplet scalars, eq. (3.6) simplifies to

$$
\begin{aligned}
k^{\zeta} & =0 \\
k_{z} & =\frac{i}{2}\left(t_{n+1}^{n+1}-t_{n}^{n}\right), \\
\hat{k}^{v^{i}} & =i\left(t^{i}{ }_{j} v^{j}-v^{i} t^{n+1}{ }_{n+1}\right), \\
\hat{k}^{u_{i}} & =i\left(t_{n+1}^{n+1} u_{i}-t^{j}{ }_{i} u_{j}-u_{i}\left(t_{n+1}^{n+1}-t_{n}^{n}\right)\right) .
\end{aligned}
$$

\footnotetext{
${ }^{19}$ Here as well as in the following section on conifold transitions we do not assume that an adapted parametrization of the Kähler cone (where $h^{I}>0$ ) has been chosen.
} 
Equating this expression to the Killing vector proposed in (4.17) yields that $t^{i}{ }_{j}=$ $a_{k} \delta^{i}{ }_{j}$ is diagonal, where the $N+1$ real constants $a_{k}$ are determined by the following system of equations

$$
\begin{aligned}
& a_{1}=t_{n+1}^{n+1} \quad, \quad-a_{\alpha}+t_{n+1}^{n+1}=1, \quad t_{n+1}^{n+1}=t_{n}^{n}, \\
& a_{1}+\sum_{\alpha=2}^{N+1} a_{\alpha}+t_{n}^{n}+t_{n+1}^{n+1}=0 .
\end{aligned}
$$

The last equation arises from the condition that $t$ should be traceless. This set of equations has the unique solution:

$$
t_{1}^{1}=t_{n}^{n}=t_{n+1}^{n+1}=\frac{N}{N+3}, \quad t_{2}^{2}=\ldots=t_{N+1}^{N+1}=-\frac{3}{N+3} .
$$

Hence the gauge generator $t_{\text {gauge }}$ is uniquely determined,

$$
t_{\text {gauge }}=\operatorname{diag}[\frac{N}{N+3}, \underbrace{-\frac{3}{N+3}, \ldots,-\frac{3}{N+3}}_{N \text { times }}, \frac{N}{N+3}, \frac{N}{N+3}] \text {. }
$$

Observe that in the case $N=1$, this is exactly the generator (3.9) of our example.

In the next step we calculate the moment map for this isometry by substituting $t_{\text {gauge }}$ into eq. (3.8). Taking linear combinations $\hat{\mu}^{1}=\hat{\mu}^{+}+\hat{\mu}^{-}, \hat{\mu}^{2}=-i\left(\hat{\mu}^{+}-\hat{\mu}^{-}\right)$ and using the definition (3.4) to simplify the resulting expressions, we obtain the following $S U(2)$ triplet of moment maps:

$$
\hat{\mu}_{\text {gauge }}^{r}=\left[\begin{array}{c}
\frac{i}{2 \phi_{+}^{1 / 2} \phi_{-}^{1 / 2}}\left(v^{\alpha} u_{\alpha}-\bar{v}^{\alpha} \bar{u}_{\alpha}\right) \\
\frac{1}{2 \phi_{+}^{1 / 2} \phi_{-}^{1 / 2}}\left(v^{\alpha} u_{\alpha}+\bar{v}^{\alpha} \bar{u}_{\alpha}\right) \\
\frac{1}{2 \phi_{-}}\left(\bar{u}_{\alpha} u_{\alpha}\right)-\frac{1}{2 \phi_{+}}\left(\bar{v}^{\alpha} v^{\alpha}\right)
\end{array}\right]
$$

The gauging of this isometry exactly proceeds as in the example of the previous section. 
In order to complete the construction of our In-picture Lagrangians we still have to calculate the scalar potential. For this purpose we first derive the superpotential $\mathcal{W}(2.21)$. The moment map $P^{r}$ is given by

$$
P^{r}=\frac{1}{2} h^{\star} \hat{\mu}_{\text {gauge }}^{r}(q)
$$

Substituting in the explicit form of $\hat{\mu}_{\text {gauge }}^{r}$, the superpotential $\mathcal{W}$ becomes

$$
\mathcal{W}=\frac{1}{\sqrt{6}} h^{\star}\left(\left(\frac{1}{2 \phi_{-}}\left(\bar{u}_{\alpha} u_{\alpha}\right)-\frac{1}{2 \phi_{+}}\left(\bar{v}^{\alpha} v^{\alpha}\right)\right)^{2}+\frac{1}{\phi_{+} \phi_{-}}\left(\bar{v}^{\alpha} \bar{u}_{\alpha}\right)\left(v^{\alpha} u_{\alpha}\right)\right)^{1 / 2}
$$

Looking at $Q^{r}$ defined in $(2.22)$, we see that

$$
Q^{r}=\sqrt{\frac{2}{3}} \frac{P^{r}}{\mathcal{W}}=\frac{\hat{\mu}_{\text {gauge }}^{r}(q)}{\left(\hat{\mu}_{\text {gauge }}^{s}(q) \hat{\mu}_{\text {gauge }}^{s}(q)\right)^{1 / 2}}
$$

is independent of the vector multiplet scalar fields and satisfies the condition $\partial_{x} Q^{r}=$ 0 . Hence the scalar potential can be expressed in terms of the superpotential and takes the form (2.23).

\section{Calculating the mass matrix}

After constructing the effective Lagrangian which includes the transition states for a generic flop transition, we now check that the masses of the scalar fields satisfy the conditions arising from the microscopic theory. Similar to the $\mathbb{F}_{1}$-model example, the only solution to the eqs. $(2.25)$ is given by setting all transition states to zero. ${ }^{20}$ The vacuum expectation values of the vector multiplet scalars and the universal hypermultiplet are not determined. Thus the manifold $\mathcal{M}_{\text {Coul }}$ of supersymmetric

\footnotetext{
${ }^{20}$ The existence of further critical points of $\mathbb{V}$ depends on the explicit choice of vector multiplet scalar manifold and is therefore not addressed here.
} 
vacua of our theory again corresponds to a Coulomb branch

$$
\mathcal{M}_{\text {Coul }}= \begin{cases}v^{\alpha}=u_{\alpha}=0, & \alpha=2, \ldots, N+1 \\ v^{1}, u_{1}, \phi^{x}, & \text { not determined }\end{cases}
$$

Substituting $\mathcal{M}_{\text {Coul }}$ into the superpotential, we find that $\mathcal{W}$ vanishes identically. Hence we have $\left.\mathbb{V}(\phi, q)\right|_{\mathcal{M}_{\text {Coul }}}=0$ and the vacuum is Minkowski. This is in complete analogy to our analysis in the previous subsection.

We will now calculate the mass matrix (4.13) for our Lagrangian. In this case it is more convenient to start from the scalar potential in the form (2.20):

$$
\mathbb{V}(\phi, q)=-4 P^{r} P^{r}+2 g^{x y} P_{x}^{r} P_{y}^{r}+\frac{3}{4} g_{X Y} K^{X} K^{Y}
$$

Here the first observation is that for the $P^{r}$ given in (4.23) the terms $P^{r} P^{r}$ and $g^{x y} P_{x}^{r} P_{y}^{r}$ are of fourth order in the transition states. This implies that these terms do not contribute to the mass matrix of our model since they vanish identically when taking two derivatives with respect to any scalar field and restricting to $\mathcal{M}_{\text {Coul }}$ afterwards. Hence the masses of our fields are solely generated by the last term in eq. (4.27).

In the next step we show that the vector multiplet scalar fields $\phi^{x}$ are massless. The matrix

$$
\mathcal{M}_{\Lambda \Sigma}:=\left.\partial_{\Lambda} \partial_{\Sigma}\left(\frac{3}{4} \mathrm{~g}^{2} g_{X Y} K^{X} K^{Y}\right)\right|_{\mathcal{M}_{\text {Coul }}}
$$

has non-trivial entries if and only if both $\Lambda$ and $\Sigma$ take values in the hypermultiplet sector. To see this, we expand $K^{X}=h^{\star}(\phi) k_{\text {gauge }}^{X}(q)$ and note that $k_{\text {gauge }}^{X}(q)$ vanishes when restricted to $\mathcal{M}_{\text {Coul }}$. This implies $\mathcal{M}_{\Lambda \Sigma}$ is only non-trivial if there is one derivative acting on each of the Killing vectors $k_{\text {gauge }}^{X}(q)$. Since $g^{\Xi \Lambda}=g^{X Y} \oplus g^{x y}$ is the direct sum of the hypermultiplet and vector multiplet inverse metrics, we find that non-trivial entries of the mass matrix (4.13) may occur in hypermultiplet sector only. This establishes that the vector multiplet scalars $\phi^{x}$ are massless. 
Thus we restrict our analysis to the case where both $\Lambda$ and $\Sigma$ take values in the hypermultiplet sector and calculate the masses of the hypermultiplets. Only terms where one of the derivatives acts on each Killing vector contribute to $\mathcal{M}_{X Y}$ :

$$
\mathcal{M}_{X Y}=\left.\frac{3}{2} \mathrm{~g}^{2}\left(h^{\star}\right)^{2} g_{W Z} \partial_{X} k_{\text {gauge }}^{W} \partial_{Y} k_{\text {gauge }}^{Z}\right|_{\mathcal{M}_{\text {Coul }}}
$$

The actual calculation of $\mathcal{M}_{X Y}$ proceeds in two steps. We first calculate the matrix $K_{X}^{Y}:=\left.\partial_{X} k_{\text {gauge }}^{Y}(q)\right|_{\mathcal{M}_{\text {Coul }}}$. With respect to the basis

$$
q^{X}=\left\{v^{1}, \ldots, v^{N+1}, \bar{v}^{1}, \ldots, \bar{v}^{N+1}, u_{1}, \ldots, u_{N+1}, \bar{u}_{1}, \ldots, \bar{u}_{N+1}\right\}
$$

$K_{X}^{Y}$ is diagonal and has the following form:

$$
K_{X}^{Y}=\operatorname{diag}[0, \underbrace{-i, \ldots,-i}_{N \text { times }}, 0, \underbrace{i, \ldots, i}_{N \text { times }}, 0, \underbrace{i, \ldots, i}_{N \text { times }}, 0, \underbrace{-i, \ldots,-i}_{N \text { times }}] \text {. }
$$

In the second step we calculate $\left.g_{X Y}(q)\right|_{\mathcal{M}_{\text {Coul }}}$ by restricting the general expression for $g_{X Y}(q)$ obtained from eq. (3.3) to $\mathcal{M}_{\text {Coul }}{ }^{21}$ We find that all blocks appearing in $\left.g_{X Y}(q)\right|_{\mathcal{M}_{\text {Coul }}}$ are diagonal:

$$
\begin{array}{ll}
g_{v_{1} \bar{v}_{1}}=\frac{1}{2 \phi_{+}^{2} \phi_{-}^{2}}\left(1-\bar{u}_{1} u_{1}\left(1-\bar{v}^{1} v^{1}\right)^{2}\right), & g_{v^{\alpha} \bar{v}^{\beta}}=\frac{1}{2 \phi_{+}} \delta_{\alpha \beta} \\
g_{u_{1} \bar{u}_{1}}=\frac{1}{2 \phi_{-}^{2}}\left(1-\bar{v}^{1} v^{1}\right), & g_{u_{\alpha} \bar{u}_{\beta}}=\frac{1}{2 \phi_{-}} \delta^{\alpha \beta} \\
g_{\bar{v}^{1} u_{1}}=-\frac{1}{2 \phi_{-}^{2}}\left(\bar{u}_{1} v^{1}\right) . &
\end{array}
$$

Here and in the following $\phi_{+}$and $\phi_{-}$are understood to be restricted to $\mathcal{M}_{\text {Coul }}$. The matrix $\mathcal{M}_{X Y}$ can now be computed from

$$
\mathcal{M}_{X Y}=\frac{3}{2} \mathrm{~g}^{2}\left(h^{\star}\right)^{2}\left[K g K^{\mathrm{T}}\right]
$$

\footnotetext{
${ }^{21}$ Recall that the components of $G_{X Y}(q)$ are minus the components of $g_{X Y}(q)$.
} 
Explicitly, we find

$$
\mathcal{M}_{X Y}=\left[\begin{array}{llll}
0 & A & 0 & 0 \\
A & 0 & 0 & 0 \\
0 & 0 & 0 & B \\
0 & 0 & B & 0
\end{array}\right]
$$

with $A$ and $B$ being the following $(N+1) \times(N+1)$-dimensional block matrices:

$$
A=\frac{3}{4 \phi_{+}} \mathrm{g}^{2}\left(h^{\star}\right)^{2} \operatorname{diag}[0, \underbrace{1, \ldots, 1}_{N \text { times }}], \quad B=\frac{3}{4 \phi_{-}} \mathrm{g}^{2}\left(h^{\star}\right)^{2} \operatorname{diag}[0, \underbrace{1, \ldots, 1}_{N \text { times }}] \text {. }
$$

Finally we need to calculate the inverse metric $g^{X Y}$, restricted to $\mathcal{M}_{\text {Coul }}$, by inverting $\left.g_{X Y}\right|_{\mathcal{M}_{\text {Coul }}}$ given in (4.32). The resulting inverse metric is again of the structure (3.2) with block diagonal entries. The only non-zero components are given by

$$
\begin{array}{ll}
g^{v^{1} \bar{v}^{1}}=2 \phi_{+}^{2} \phi_{-}, & g^{v^{\alpha} \bar{v}^{\beta}}=2 \phi_{+} \delta^{\alpha \beta} \\
g^{u_{1} \bar{u}_{1}}=2 \frac{\phi_{-}}{\phi_{+}}\left(1-\bar{u}_{1} u_{1}\left(1-\bar{v}^{1} v^{1}\right)^{2}\right), & g^{u_{\alpha} \bar{u}_{\beta}}=2 \phi_{-} \delta^{\alpha \beta} \\
g^{v^{1} \bar{u}_{1}}=2 \phi_{+} \phi_{-}\left(\bar{u}_{1} v^{1}\right) &
\end{array}
$$

The hypermultiplet masses are given by the eigenvalues of the mass matrix

$$
\mathcal{M}_{Y}^{X}=\left.g^{X Z} \mathcal{M}_{Z Y}\right|_{\mathcal{M}_{\text {Coul }}}
$$

Using the results (4.34) and (4.36), we find that the resulting matrix is diagonal

$$
\mathcal{M}_{Y}^{X}=\left(m_{\text {Flop }}\right)^{2}[0, \underbrace{1, \ldots, 1}_{N \text { times }}, 0, \underbrace{1, \ldots, 1}_{N \text { times }}, 0, \underbrace{1, \ldots, 1}_{N \text { times }}, 0, \underbrace{1, \ldots, 1}_{N \text { times }}]_{Y}^{X}
$$

where $\left(m_{\text {Flop }}\right)^{2}=\frac{3}{2}\left(h^{\star}\right)^{2} \mathrm{~g}^{2}$. This result explicitly shows that our Lagrangian contains one massless hypermultiplet, given by the complex fields $v^{1}, u_{1}$. This multiplet corresponds to the universal hypermultiplet. The transition states $v^{\alpha}, u_{\alpha}$ all acquire 
the same mass

$$
m_{\text {Flop }}=\sqrt{\frac{3}{2}} \mathrm{~g} h^{\star}
$$

It is proportional to the volume of the flopped cycle, $h^{\star}$, as required by the underlying microscopic theory. Comparing (4.39) to eq. (2.36) we find that the gauge coupling constant $\mathrm{g}$ is again set by (4.16).

This result concludes the construction of the In-picture Lagrangian for a generic flop transition. We find that after fixing the hypermultiplet scalar manifold to be $X(N+1)$, the hypermultiplet sector of the resulting action is uniquely determined in terms of the microscopic theory. We further note that in order to calculate the mass matrix, we did not need to specify the details of the vector multiplet sector. Hence the analysis in this section can be used to model any flop transition where $N$ charged hypermultiplets become massless. In the case where $N=1$ these results exactly match the ones found in the explicit example given in subsection 4.1.

\section{The conifold transition}

After deriving the In-picture LEEA for a general flop transition we now turn to the In-picture description of a general conifold transition. In analogy to the flop we will proceed by first constructing the Lagrangian before showing that it gives rise to the vacuum structure and mass matrix intrinsic to the transition. The new feature of these Lagrangians is that, besides the Coulomb branch familiar from the flop, they have a second supersymmetric vacuum branch, a Higgs branch.

In terms of the algebraic eqs. (2.25) describing the supersymmetric vacuum structure of the Lagrangians this setup can be understood as follows. Suppose we gauge an isometry where we can fix some of the hypermultiplet scalars to obtain $P_{I}^{r}(q)=0$ while the corresponding Killing vector $K_{I}^{X}(q)$ remains non-zero. Having $P_{I}^{r}(q)=0$ suffices to meet the first condition in $(2.25) .{ }^{22}$ In order to satisfy the

\footnotetext{
${ }^{22}$ In terms of four-dimensional super-Yang-Mills theory the conditions $P_{I}^{1}(q)=P_{I}^{2}(q)=0$ and $P_{I}^{3}(q)=0$ correspond to $F$ - and $D$-flatness, respectively [65].
} 
second equation, $h^{I}(\phi) K_{I}^{X}(q)=0$, we then have the choice to either fix the remaining hypermultiplet scalars such that $K_{I}^{X}(q)=0$ or the vector multiplet scalars such that $h^{I}(\phi)=0$. The first choice corresponds to the Coulomb branch, while the second one gives rise to the Higgs branch. The intersection locus where both $K_{I}^{X}(q)=0$ and $h^{I}(\phi)=0$ are fulfilled is the conifold locus.

In order to identify the correct isometries whose gauging describes the charged states arising in the conifold transition we turn to the corresponding microscopic description. The analysis of subsection 2.3 indicates that we should look for a set of Killing vectors $\alpha=1, \ldots, N-1$ of the form ${ }^{23}$

$$
\begin{aligned}
& \hat{k}_{\alpha}^{v}=i\left[0, v^{2}, 0, \ldots,-v^{\alpha+2}, 0, \ldots, 0\right]^{\mathrm{T}} \\
& \hat{k}_{\alpha}^{u}=i\left[0,-u^{2}, 0, \ldots, u^{\alpha+2}, 0, \ldots, 0\right]^{\mathrm{T}}
\end{aligned}
$$

where the entries ' $-v^{\alpha+2}$ ' and ' $u^{\alpha+2}$ ' occur at the $\alpha+2$ position. The components of $\hat{k}_{\alpha}^{X}$ with respect to $\partial_{\bar{v}^{i}}$ and $\partial_{\bar{u}_{i}}$ are obtained from $\hat{k}_{\alpha}^{v}$ and $\hat{k}_{\alpha}^{u}$ by complex conjugation. Here we have chosen our hypermultiplets such that $v^{1}, u_{1}$ parametrize the universal hypermultiplet, $v^{2}, u_{2}$ arise from the M2-brane wrapping the cycle $\mathcal{C}_{1}=-\sum_{\alpha=2}^{N} \mathcal{C}_{\alpha}$, and the remaining hypermultiplets $v^{\alpha+1}, u_{\alpha+1}$ correspond to the M2-branes wrapping the cycles $\mathcal{C}_{\alpha}, \alpha=2, \ldots, N$, respectively. The set of Killing vectors (5.1) mutually commutes, $\left[\hat{k}_{\alpha}, \hat{k}_{\beta}\right]=0$, so that the hypermultiplets are charged with respect to a subgroup $U(1)^{N-1} \subset U(1)^{n_{V}+1}$. Equation (5.1) further indicates that we need at least two charged hypermultiplets in order to describe a conifold transition.

\subsection{The minimal Lagrangian}

Before constructing the In-picture LEEA describing the general conifold transition, let us first illustrate the essential features of the transition by considering a minimal

\footnotetext{
${ }^{23}$ Here we consider the case where $N$ holomorphic curves satisfying $r=1$ homology relations are contracted and all the neutral hypermultiplets except the universal hypermultiplet have been truncated.
} 
model containing one vector and two hypermultiplets. The general analysis given above implies that in this case both hypermultiplets must correspond to transition states, i.e., we are in a situation where the universal hypermultiplet has also been truncated. The vector multiplet sector is taken to be the most general one vector multiplet manifold discussed in appendix A while the hypermultiplet sector is given by $X(2)$.

\section{Constructing the action}

Our first task is then to identify a suitable gauge connection $A_{\mu}^{\star}$. The Higgs branch condition $h^{\star}\left(\phi_{c}\right) K_{\star}^{X}\left(q_{c}\right)=0, K_{\star}^{X}\left(q_{c}\right) \neq 0$ requires that $h^{\star}(\phi)$ must have a zero for some value $\phi$ inside the vector multiplet scalar manifold. The discussion in appendix A then fixes $h^{\star}(\phi)=h^{1}(\phi)$ since $h^{0}(\phi)$ does not have this property. Second we need an analog of the isometry (5.1) for this model. Truncating the universal hypermultiplet and assigning negative and positive $U(1)$ charge with respect to $A_{\mu}^{\star}$ to $u_{1}$ and $u_{2}$, respectively, implies that the corresponding Killing vector is given by eq. (3.12) with its moment map being (3.15).

The gauging of the general supergravity Lagrangian (2.1) proceeds completely analogous to the case of the flop transition. Based on the previous discussion we set

$$
P^{r}(\phi, q):=\frac{1}{2} h^{1}(\phi) \mu_{\mathrm{Cf}}^{r}(q), \quad K^{X}(\phi, q):=h^{1}(\phi) k_{\mathrm{Cf}}^{X}(q)
$$

with $k_{\mathrm{Cf}}^{X}$ and $\mu_{\mathrm{Cf}}^{r}$ given in (3.12) and (3.15), respectively. Substituting $P^{r}(\phi, q)$ given above into the superpotential (2.21), we obtain

$$
\begin{array}{r}
\mathcal{W}=\frac{h^{1}}{\sqrt{6}}\left\{\left(\frac{1}{2 \phi_{+}}\left(v^{1} \bar{v}^{1}-v^{2} \bar{v}^{2}\right)-\frac{1}{2 \phi_{-}}\left(u_{1} \bar{u}_{1}-u_{2} \bar{u}_{2}\right)\right)^{2}\right. \\
\left.+\frac{1}{\phi_{+} \phi_{-}}\left(v^{1} u_{1}-v^{2} u_{2}\right)\left(\bar{v}^{1} \bar{u}_{1}-\bar{v}^{2} \bar{u}_{2}\right)\right\}^{1 / 2} .
\end{array}
$$

Following the calculation $(4.23)-(4.25)$ we establish that $Q^{r}$ defined in eq. $(2.22)$ 
is independent of the vector multiplet scalar. This implies that the scalar potential $\mathbb{V}(\phi, q)$ is determined by its stability form $(2.23)$.

\section{Vacua and mass matrices}

The supersymmetric vacua of this potential are determined by the conditions (2.25). Looking at the moment map (3.15) reveals that $\hat{\mu}_{\mathrm{Cf}}^{r}$ vanishes if and only if $v^{1}=$ $v^{2}, u_{1}=u_{2}$. Restricting the Killing vector (3.12) to this subset we find that the resulting expression is non-vanishing. The second condition in (2.25) can then be met by either setting the hypermultiplet scalars to zero, which corresponds to the Coulomb branch

$$
\mathcal{M}_{\text {Coul }}=\left\{v^{1}=u_{1}=v^{2}=u_{2}=0, \phi \text { undetermined }\right\}
$$

or fixing $\phi=0$ which yields the Higgs branch

$$
\mathcal{M}_{\mathrm{Higgs}}=\left\{v^{1}=v^{2}=: v, u_{1}=u_{2}=: u, \phi=0\right\}
$$

On the latter, the non-trivial vacuum expectation value of the hypermultiplet scalars spontaneously breaks the $U(1)$ gauge group. Restricting the superpotential (5.3) to these vacua, we find that the resulting expression vanishes. This implies that these vacua are Minkowski, establishing that our model has the correct vacuum structure to describe a conifold transition.

To complete our discussion, we also calculate the masses of the fields on the two vacuum branches. In analogy to eq. (4.10), we take the coordinates on $\mathcal{M}_{\mathrm{HM}} \times \mathcal{M}_{\mathrm{VM}}$ to be

$$
\phi^{\Lambda}=\left\{v^{1}, v^{2}, \bar{v}^{1}, \bar{v}^{2}, u_{1}, u_{2}, \bar{u}_{1}, \bar{u}_{2}, \phi\right\}
$$

The evaluation of the mass matrix (4.13) on the Coulomb branch proceeds exactly 
as in the case of a flop transition and yields

$$
\left.\mathcal{M}_{\Sigma}^{\Lambda}\right|_{\text {Coul }}=\left(m_{\text {Coul }}\right)^{2} \operatorname{diag}[1,1,1,1,1,1,1,1,0]
$$

with

$$
\left(m_{\text {Coul }}\right)^{2}=\frac{3}{2} \mathrm{~g}^{2}\left(h^{1}\right)^{2}
$$

This is exactly the same mass as the one obtained for the transition states occurring in the flop transition (4.39) which indicates that we obtained the correct BPS mass for hypermultiplets arising from M2-branes wrapping a holomorphic cycle with volume $h^{1}$.

Let us now turn to the Higgs branch. Here we have to evaluate the mass matrix for the scalar fields (4.13) and in addition obtain a mass term for the vector field $A_{\mu}^{1}$. In the latter case we note that since $\left.a_{I J}\right|_{\phi=0}=\mathbb{1}_{2 \times 2}(\mathrm{cf}$. appendix $\mathrm{A})$, the kinetic term for the vector fields has its standard form, so that the mass of $A_{\mu}^{1}$ can be read off directly from the term proportional to $\left(A_{\mu}^{1}\right)^{2}$ arising in the hypermultiplet kinetic term. Defining

$$
\left(m_{\mathrm{Higgs}}\right)^{2}=2 \mathrm{~g}^{2}\left(\frac{1}{\phi_{+}} v \bar{v}+\frac{1}{\phi_{-}} u \bar{u}\right)
$$

where $\phi_{ \pm}$is understood to be restricted to $\mathcal{M}_{\text {Higgs }}$, we find that the vector field $A_{\mu}^{1}$, the vector multiplet scalar $\phi$ and three hypermultiplet degrees of freedom acquire the mass $m_{\text {Higgs }}$ while the remaining fields stay massless. ${ }^{24}$ In terms of supersymmetry algebra representations (see for example [68]) these modes organize into one massless hypermultiplet and one long vector multiplet of mass $m_{\mathrm{Higgs}}$ containing the massive degrees of freedom as well as one massless hypermultiplet mode providing the longitudinal mode of the vector field. In this sense the vector multiplet 'eats up' one of the hypermultiplets to become a non-BPS multiplet.

In terms of CY compactifications this model has the following interpretation.

\footnotetext{
${ }^{24}$ Note that one of the massless hypermultiplet scalars has an interpretation as a Goldstone boson arising from the spontaneous broken $U(1)$ gauge symmetry.
} 
At the conifold point all fields are massless and we have one vector and two hypermultiplets. Far away from this point, however, the massive degrees of freedom are heavy and should be integrated out. In this case only the massless modes on the branches remain and we acquire one massless vector or hypermultiplet when on the Coulomb or Higgs branch, respectively. This minimal model can then be interpreted as connecting two $\mathrm{CY}$ compactifications $X$ and $\tilde{X}$ associated with the Coulomb and the Higgs branch. Their Hodge numbers differ by $h^{1,1}(\tilde{X})=h^{1,1}(X)-1$ and $h^{1,2}(\tilde{X})=h^{1,2}(X)+1$ in agreement with the general formula $(2.35)$ for $N=2, r=1$. In section 8 we will demonstrate that this transition from the Coulomb to the Higgs branch can indeed be realized dynamically.

\subsection{The Lagrangian for general conifold transitions}

After working out the minimal Lagrangian describing a conifold transition we will now generalize this setup to the case where $N$ charged hypermultiplets become massless at the transition locus. When including the neutral universal hypermultiplet, the hypermultiplet sector of the corresponding Lagrangian contains $N+1$ hypermultiplets and thus will be modelled by $X(N+1)$. In contrast to the actions describing the general flop transition we also need to specify an explicit vector multiplet sector for the conifold Lagrangian so that we can determine the mass matrix on the Higgs branch. In this sector we will use the family of very special real manifolds discussed in appendix $\mathrm{B}$ with $n_{V} \geq N-1$ vector multiplets. Even though these manifolds are not directly related to an exact In-picture vector multiplet sector arising from a CY compactification, this ansatz is general enough that it can readily be adjusted to any particular conifold transition by substituting the corresponding In-picture vector multiplet sector obtained from eq. (2.37). 


\section{Constructing the action}

Our first task in constructing the Lagrangian of our model is to determine the generators $t_{\alpha} \in s u(N+1,2)$ which give rise to the isometries (5.1). For this purpose we first restrict the expression for a general Killing vector on $X(N+1),(3.6)$, to the subset of Killing vectors which are linear in the hypermultiplet scalar fields given by eq. (4.18). Equating this expression with the Killing vectors (5.1) and imposing the condition that $t_{\alpha}$ is traceless leads to an overdetermined system of equations from which the generators $t_{\alpha}$ can be determined. In the case of the Killing vectors (5.1) we find the solution

$$
t_{\alpha}=\operatorname{diag}[0,1,0, \ldots,-1,0, \ldots, 0]
$$

where the entry ' -1 ' sits at the $\alpha+2$ position. Hence the Killing vectors (5.1) are indeed isometries of $X(N+1)$.

In the next step we calculate the moment maps $\hat{\mu}_{\alpha}^{r}$ of these isometries. Substituting the generators $t_{\alpha}$ into eq. (3.8) and taking the linear combinations $\hat{\mu}^{1}=\hat{\mu}^{+}+\hat{\mu}^{-}$, $\hat{\mu}^{2}=-i\left(\hat{\mu}^{+}-\hat{\mu}^{-}\right)$we obtain

$$
\hat{\mu}_{\alpha}^{r}=\left[\begin{array}{c}
-\frac{i}{2 \phi_{+}^{1 / 2} \phi_{-}^{1 / 2}}\left(v^{2} u_{2}-v^{\alpha+2} u_{\alpha+2}-\bar{v}^{2} \bar{u}_{2}+\bar{v}^{\alpha+2} \bar{u}_{\alpha+2}\right) \\
-\frac{1}{2 \phi_{+}^{1 / 2} \phi_{-}^{1 / 2}}\left(v^{2} u_{2}-v^{\alpha+2} u_{\alpha+2}+\bar{v}^{2} \bar{u}_{2}-\bar{v}^{\alpha+2} \bar{u}_{\alpha+2}\right) \\
-\frac{1}{2 \phi_{-}}\left(u_{2} \bar{u}_{2}-u_{\alpha+2} \bar{u}_{\alpha+2}\right)+\frac{1}{2 \phi_{+}}\left(v^{2} \bar{v}^{2}-v^{\alpha+2} \bar{v}^{\alpha+2}\right)
\end{array}\right] .
$$

In order to complete the construction of the Lagrangian, we then have to gauge these isometries. Following our previous discussion we need $N-1$ gauge fields $A_{\mu}^{\alpha}$ whose corresponding scalar fields $h^{\alpha}(\phi)$ vanish at some subset of the vector multiplet scalar manifold. By virtue of eq. (B.5) this condition is satisfied for all $h^{I}(\phi), I=1, \ldots, n_{V}$, but not for $h^{0}(\phi)$. Without loss of generality we then choose the gauge fields $A_{\mu}^{\alpha}, \alpha=1, \ldots, N-1$, to serve as the gauge connections for the Killing vectors. 
The gauging of the Lagrangian proceeds analogous to the one for the flop and we set

$$
P^{r}=\frac{1}{2} h^{\alpha}(\phi) \hat{\mu}_{\alpha}^{r}(q), \quad K^{X}=h^{\alpha}(\phi) k_{\alpha}^{X}(q),
$$

with the sets $k_{\alpha}^{X}(q)$ and $\hat{\mu}_{\alpha}^{r}(q)$ given in (5.1) and (5.11), respectively. The scalar potential of this Lagrangian is determined by substituting these relations, the hypermultiplet metric obtained from eq. (3.2) and the inverse vector multiplet scalar metric (B.7) into

$$
\mathbb{V}(\phi, q)=-4 P^{r} P^{r}+2 g^{x y} P_{x}^{r} P_{y}^{r}+\frac{3}{4} g_{X Y} K^{X} K^{Y}
$$

This completes the construction of the Lagrangian. Let us remark that rewriting $\mathbb{V}(\phi, q)$ in terms of the superpotential $(2.21)$ is not helpful in this case as it is simpler to determine the vacuum structure and mass matrices of the Lagrangian in terms of the moment maps and the potential (5.13).

\section{Vacua and mass matrices}

We now check whether the supersymmetric vacuum structure and the mass matrices of the Lagrangians constructed above agree with the specifications coming from the microscopic theory. Determining the vacuum structure again utilizes the algebraic eqs. (2.25) and proceeds completely analogous to the one for the minimal model. Looking at the set of moment maps (5.11) reveals that they vanish if and only if all charged hypermultiplets have the same value, i.e., if $v^{2}=\ldots=v^{N+1}=v$ and $u_{2}=\ldots=u_{N+1}=u$. To solve the second condition in (2.25) we then have the choice to either fix $v=u=0$, leading to the Coulomb branch

$$
\mathcal{M}_{\text {Coul }}=\left\{v^{2}=\ldots=v^{N+1}=0, u_{2}=\ldots=u_{N+1}=0\right\}
$$


or to set $\phi^{\alpha}=0 \leftrightarrow h^{\alpha}(\phi)=0$ which corresponds to the Higgs branch

$$
\mathcal{M}_{\mathrm{Higgs}}=\left\{v=v^{2}=\ldots=v^{N+1}, u=u_{2}=\ldots=u_{N+1}, \phi^{\alpha}=0\right\}
$$

The scalars not present in these equations are not fixed by the vacuum conditions and correspond to flat directions. We further note that the non-trivial value of the hypermultiplet scalars on the Higgs branch breaks the $U(1)^{N-1} \subset U(1)^{n_{V}+1}$ spontaneously. Substituting the conditions of vanishing $\hat{\mu}_{\alpha}^{r}$ and $K^{X}$ into the potential (5.13) shows that these vacua are Minkowski so that we obtain the correct vacuum structure expected for a conifold transition.

Our next task is to calculate the mass matrices for these vacuum branches. As in the case of the general flop transition, we start with the scalar potential in the form (5.13). We first analyze the Coulomb branch before turning to the Higgs branch.

In the first step of determining the mass matrix (4.13) we check which terms of the potential give a non-zero contribution. On the Coulomb branch we can use the arguments given in the case of the general flop (see below eq. (4.27)) to establish that only the hypermultiplet scalars obtain a mass and that we can restrict ourselves to evaluating the matrix

$$
\mathcal{M}_{X Y}=\left.\frac{3}{2} \mathrm{~g}^{2} h^{\alpha} h^{\beta} g_{W Z}\left(\partial_{X} k_{\alpha}^{W}\right)\left(\partial_{Y} k_{\beta}^{Z}\right)\right|_{\mathcal{M}_{\mathrm{Coul}}}
$$

For this purpose we first compute $K_{X}^{Y}:=h^{\alpha} \partial_{X} k_{\alpha}^{Y}(q)$. Using the basis (4.30) the resulting matrix is diagonal,

$$
K_{X}^{Y}=i \operatorname{diag}[H,-H,-H, H]
$$

with

$$
H=\operatorname{diag}\left[0,-\sum h^{\alpha}, h^{1}, \ldots, h^{N-1}\right]
$$

Next we restrict the hypermultiplet scalar metric $g_{X Y}(q)$ to the Coulomb branch, 
which gives rise to the non-zero entries (4.32). The matrix $\mathcal{M}_{X Y}$ is then calculated via eq. (4.33) and has again the form (4.34) with the block matrices $A$ and $B$ now given by

$$
\begin{aligned}
& A=\frac{3}{4 \phi_{+}} \mathrm{g}^{2}\left[0,\left(\sum h^{\alpha}\right)^{2},\left(h^{1}\right)^{2}, \ldots,\left(h^{N-1}\right)^{2}\right] \\
& B=\frac{3}{4 \phi_{-}} \mathrm{g}^{2}\left[0,\left(\sum h^{\alpha}\right)^{2},\left(h^{1}\right)^{2}, \ldots,\left(h^{N-1}\right)^{2}\right] .
\end{aligned}
$$

Raising one index of $\mathcal{M}_{X Y}$ with the inverse hypermultiplet metric restricted to the Coulomb branch (4.36) we obtain the matrix $\mathcal{M}_{Y}^{X}$ encoding the hypermultiplet scalar masses. Explicitly we find the masses for the universal hypermultiplet $v^{1}, u_{1}$, the hypermultiplet $v^{2}, u_{2}$ arising from the wrapped cycle $\mathcal{C}_{1}=-\sum_{\alpha=2}^{N} \mathcal{C}_{\alpha}$ and the other hypermultiplets $v^{\alpha+1}, u_{\alpha+1}$ associated with the cycles $\mathcal{C}_{\alpha}, \alpha=2, \ldots, N$, of volume $h^{\alpha-1}$ to be

$$
m_{1}=0, \quad m_{2}=\sqrt{\frac{3}{2}} \mathrm{~g} \sum_{\alpha=1}^{N-1} h^{\alpha}, \quad \text { and } \quad m_{\alpha+1}=\sqrt{\frac{3}{2}} \mathrm{~g} h^{\alpha-1}
$$

respectively. These masses are in complete agreement with the microscopic theory, which demands that the mass of the hypermultiplets is proportional to the volume of the cycle wrapped by the corresponding M2-brane. Comparing these masses to the BPS mass formula (2.36) fixes g to the value (4.16). This input pins down the remaining freedom of the In-picture LEEA in terms of the microscopic data.

Let us now turn to the Higgs branch. Here we do not have a prediction for the masses of the massive states in terms of the microscopic description. But since the data on the Coulomb branch together with the charge assignment (5.1) completely determines the Lagrangian, we can use our model to predict the masses on this branch.

We again begin our calculation by investigating which terms of the potential (5.13) contribute to the mass matrix on the Higgs branch. The first term pro- 
portional to $P^{r} P^{r}$ is of second order in both $\phi^{\alpha}$ and $\hat{\mu}_{\alpha}^{r}$ which both vanish when restricted to the Higgs branch. Hence all its second derivatives vanish when restricted to $\mathcal{M}_{\text {Higgs }}$ and there is no contribution to the mass matrix. The second term, $2 g^{x y} P_{x}^{r} P_{y}^{r}$, has orders zero and two in $\phi^{\alpha}$ and $\hat{\mu}_{\alpha}^{r}$, respectively. This indicates that this term is of relevance for the hypermultiplet mass matrix, as the only non-vanishing second derivative terms (restricted to $\mathcal{M}_{\text {Higgs }}$ ) are obtained when one derivative with respect to a hypermultiplet scalar acts on each of the moment maps. Finally the third term is of second order in $\phi^{\alpha}$ while the Killing vectors $k_{\alpha}^{X}$ do not vanish on $\mathcal{M}_{\text {Higgs }}$. Therefore this term gives rise to the vector multiplet scalar masses, as we have to take two derivatives with respect to the vector multiplet scalars to have a non-vanishing expression when restricting to $\mathcal{M}_{\mathrm{Higgs}}$.

The analysis of the supersymmetry representations [68] and the explicit example of our minimal Lagrangian show that the massive modes on this branch combine into massive long vector multiplets composed of one vector and one hypermultiplet of the same mass. In order to determine this mass it then suffices to compute the vector multiplet scalar masses arising from the third term in the potential.

We start by evaluating

$$
\mathcal{M}_{x y}=\left.\frac{3}{2} \mathrm{~g}^{2} g_{X Y}\left(\partial_{x} h^{\alpha}(\phi) k_{\alpha}^{X}\right)\left(\partial_{y} h^{\beta}(\phi) k_{\beta}^{Y}\right)\right|_{\mathcal{M}_{\mathrm{Higgs}}}
$$

To this end we first calculate the $\left(n_{V}\right) \times 4(N+1)$ dimensional matrix $K_{x}{ }^{X}:=$ $\partial_{x} h^{\alpha}(\phi) k_{\alpha}^{X}$. Taking the basis (4.30) for the hypermultiplets and using the definition (B.5) for $h^{I}(\phi)$ we find

$$
K_{x}{ }^{X}=h^{0}\left[\begin{array}{cccc}
-i v^{2} \mathcal{Q} & i \bar{v}^{2} \mathcal{Q} & i u_{2} \mathcal{Q} & -i \bar{u}_{2} \mathcal{Q} \\
0 & 0 & 0 & 0
\end{array}\right]
$$


where $\mathcal{Q}$ denotes the $(N-1) \times(N+1)$ matrix $^{25}$

$$
\mathcal{Q}=\left[\begin{array}{cc|c}
0 & -1 & \\
\vdots & \vdots & \mathbb{1}_{(N-1) \times(N-1)} \\
0 & -1 &
\end{array}\right]
$$

and ' 0 ' is short for the $\left(n_{V}-N+1\right) \times(N+1)$ dimensional zero matrix.

It is then straightforward to calculate $\mathcal{M}_{x y}=\left.\frac{3}{2} \mathrm{~g}^{2}\left[K g K^{\mathrm{T}}\right]_{x y}\right|_{\mathcal{M}_{\mathrm{Higgs}}}$ to be

$$
\mathcal{M}_{x y}=3 \mathrm{~g}^{2}\left(h^{0}\right)^{2} \operatorname{diag}[\Pi, 0] .
$$

Here $\Pi$ denotes the $(N-1) \times(N-1)$ matrix

$$
\Pi=|v|^{2} \mathcal{Q} g_{v \bar{v}} \mathcal{Q}^{\mathrm{T}}+|u|^{2} \mathcal{Q} g_{u \bar{u}} \mathcal{Q}^{\mathrm{T}}-v \bar{u} \mathcal{Q} g_{\bar{u} v} \mathcal{Q}^{\mathrm{T}}-u \bar{v} \mathcal{Q} g_{u \bar{v}} \mathcal{Q}^{\mathrm{T}}
$$

where $v, u$ are the values of the hypermultiplet scalars on the Higgs branch and $g_{\bar{v} v}$, $g_{\bar{u} u}, g_{\bar{u} v}$ and $g_{u \bar{v}}$ denote the block matrices appearing in the hypermultiplet scalar metric $g_{X Y}(q)$. Introducing $M:=\mathcal{Q} g \mathcal{Q}^{\mathrm{T}}$ with $g$ representing the blocks of $g_{X Y}(q)$ we find that there is a simple relation between the components of $M$ and $g$

$$
m_{\alpha \beta}=g_{22}-g_{(\alpha+2) 2}-g_{2(\beta+2)}+g_{(\alpha+2)(\beta+2)} .
$$

Evaluating this expression on $\mathcal{M}_{\text {Higgs }}$, we find $M=0$ if $g=g_{u \bar{v}}$ or $g=g_{\bar{u} v}$ while for $g=g_{v \bar{v}}$ and $g=g_{u \bar{u}}$ we obtain

$$
m_{\alpha \beta}=\frac{1}{2 \phi_{+}}\left(1+\delta_{\alpha \beta}\right) \quad \text { and } \quad m_{\alpha \beta}=\frac{1}{2 \phi_{-}}\left(1+\delta_{\alpha \beta}\right)
$$

respectively. This allows us to write down $\mathcal{M}_{x y}$ explicitly. Its non-zero components

\footnotetext{
${ }^{25}$ This matrix is the charge matrix of the $N+1$ hypermultiplets with respect to the $U(1)^{N-1}$ gauge connections $A_{\mu}^{\alpha}$.
} 
read

$$
\mathcal{M}_{\alpha \beta}=\frac{3}{2} \mathrm{~g}^{2}\left(h^{0}\right)^{2}\left(\frac{1}{\phi_{+}}|v|^{2}+\frac{1}{\phi_{-}}|u|^{2}\right)\left(1+\delta_{\alpha \beta}\right)
$$

The mass matrix $\mathcal{M}^{x}{ }_{y}$ for the vector multiplet scalars is obtained by raising one index of $\mathcal{M}_{x y}$ using the inverse vector multiplet scalar metric restricted to the Higgs branch (B.8). Defining

$$
\left(m_{\mathrm{Higgs}}\right)^{2}:=\mathrm{g}^{2}\left(\left(h^{0}\right)^{2}-\frac{3}{2} \sum_{z=N}^{n_{V}}\left(h^{z}\right)^{2}\right)\left(\frac{1}{\phi_{+}}|v|^{2}+\frac{1}{\phi_{-}}|u|^{2}\right)
$$

where $\phi_{+}$and $\phi_{-}$are understood to be restricted to $\mathcal{M}_{\text {Higgs }}$, we find

$$
\mathcal{M}^{x}{ }_{y}=\left(m_{\text {Higgs }}\right)^{2} \operatorname{diag}\left[\mathcal{M}_{\beta}^{\alpha}, 0\right]
$$

with $\mathcal{M}_{\beta}^{\alpha}=1+\delta_{\beta}^{\alpha}$.

This result shows that all 'spectator vector multiplets' $h^{I}, I=N, \ldots, n_{V}$, which do not serve as a gauge connection for the isometries remain massless. Determining the eigenvalues of $\mathcal{M}_{\beta}^{\alpha}$ shows that the vector multiplet scalars $\phi^{\alpha}$ acquire two different masses. One scalar has mass $(N)^{1 / 2} m_{\text {Higgs }}$ while the remaining $N-2$ scalars have degenerate mass $m_{\text {Higgs }}$. Here it is interesting to observe that, in the presence of spectator vector multiplets, the mass of these states depends on both the vector and hypermultiplet degrees of freedom which points at the non-BPS nature of the corresponding long vector multiplets. We also observe that in the absence of spectator vector multiplets and for $N=2$ the general formula (5.30) agrees with the results obtained for the minimal Lagrangian of the previous subsection.

In terms of CY compactifications, these general Lagrangians have the following interpretation. On the Coulomb branch, associated with the CY compactification on $X$, we have $n_{V}$ vector and one hypermultiplets which are generically massless. At the conifold locus one obtains $N$ additional massless hypermultiplets. When going to the Higgs branch arising from the CY compactification on $\tilde{X}, N-1$ charged 
hypermultiplets are 'eaten up' by the massive vector multiplets to form long vector multiplets. We are then left with $n_{V}-(N-1)$ vector and two hypermultiplets which are generically massless. This establishes that our In-picture LEEA indeed provides a continuous description of a conifold transition where the Hodge numbers change by $h^{1,1}(\tilde{X})=h^{1,1}(X)-(N-1)$ and $h^{1,2}(\tilde{X})=h^{1,2}(X)+1$. In particular this class includes the conifold transition of the quintic studied in $[9,2]$ where the Hodge numbers of $X$ and $\tilde{X}$ are given by $h^{1,1}(X)=102, h^{1,2}(X)=0$ and $h^{1,1}(\tilde{X})=87$, $h^{1,2}(\tilde{X})=1$.

\section{Kasner cosmologies and Out-picture flops}

After constructing the In-picture LEEA describing a general flop and conifold transition we now come to the second part of this thesis and investigate the effects of the transition states on the Kasner cosmologies introduced in subsection 2.4. In this course we first turn to the Out- and In-picture description of a flop transition in this and the subsequent section, respectively, before studying cosmological solutions undergoing a conifold transition in section 8.

We begin with cosmological solutions in the Out-picture. In this case the transition states have been integrated out and the transition manifests itself in a discontinuity in the triple intersection numbers. We proceed by first deriving some general properties of such transitions which can be established without specifying the vector and hypermultiplet manifolds in subsection 6.1 before focusing upon the $\mathbb{F}_{1}$-model in subsection 6.2 .

\subsection{General properties of cosmological solutions}

We start our investigation by considering an arbitrary CY threefold and cosmological solutions which pass through a topological phase transition involving a finite number of transitions states but no change in the Hodge numbers. ${ }^{26}$ This means that we do

\footnotetext{
${ }^{26}$ This excludes the conifold transitions discussed in section 5 .
} 
admit both flop transitions with an arbitrary number of charged hypermultiplets, and type-III contractions which lead to $S U(2)$ gauge symmetry enhancement [67, $16,5,23]$. In the latter case one has two charged vector multiplets together with a number of charged hypermultiplets depending on the details of the contraction. We will show that the logarithmic scale factors $\nu(t), \alpha(t)$ and $\beta(t)$ of the metric (2.39) are smooth across any topological phase transition satisfying these assumptions. ${ }^{27}$

The only source of discontinuities in the Out-picture is the jump in the triple intersection numbers, which can be understood as a threshold effect. When integrating out the transition states one finds that the Out-picture prepotentials in the two Kähler cones differ by the amount [5, 16, 23]

$$
\Delta \mathcal{V}=\frac{1}{6}\left(\delta n_{H}-\delta n_{V}\right)\left(h^{\star}\right)^{3}
$$

Here $h^{\star}=q_{I} h^{I}$ is proportional to the volume of the collapsing cycle ${ }^{28} C^{\star}=q_{I} C^{I}$, while $\delta n_{V}$ and $\delta n_{H}$ count the vector and hypermultiplets which become massless in the transition. The transition locus corresponds to $h^{\star}=0$. By virtue of eq. (2.4) we find that $\Delta \mathcal{V}$ does not contribute to the metric $a_{I J}$ at the transition point. Hence $a_{I J}$ and also $g_{x y}$ will be continuous. But the first derivative of $a_{I J}$ with respect to $h^{\star}$ is not continuous due to the jump in the triple intersection numbers indicated by eq. (6.1). This implies that the derivative of $g_{x y}$ and therefore the Christoffel connection $\gamma_{y z}^{x}$ is discontinuous at the transition locus.

Next we impose that the function $T$ defined in (2.44) is constant. As we will see, this corresponds to a specific choice of the lapse function in our ansatz and leads to a consistent solution. If $T$ is constant the Einstein equations decouple from the

\footnotetext{
${ }^{27}$ This was also shown in [13] for the flop of a single curve.

${ }^{28}$ The $C^{I}$ are a basis of $H_{2}(X, \mathbb{Z})$, therefore $q_{I} \in \mathbb{Z}$.
} 
matter equations and can be solved analytically

$$
\begin{aligned}
\alpha(t) & =c_{1} t+c_{2}, \\
\beta(t) & =-\frac{1}{3 c_{1}}\left(3 c_{1}^{2}-T\right) t+c_{3}, \\
\nu(t) & =\frac{1}{3 c_{1}}\left(6 c_{1}^{2}+T\right) t+c_{4},
\end{aligned}
$$

where the $c_{i}$ are constants of integration. This solution does not depend on the choice of the vector and hypermultiplet scalar metric. It satisfies

$$
3 \dot{\alpha}+\dot{\beta}-\dot{\nu}=0 .
$$

We read this as a condition which fixes the lapse function such that $T$ is constant with respect to the corresponding time variable. Now we have to check whether this is consistent with the scalar equations of motion. Substituting in our result, these reduce to the standard geodesic equations with respect to the vector and hypermultiplet scalar metrics

$$
\ddot{\phi}^{x}+\gamma_{y z}^{x} \dot{\phi}^{y} \dot{\phi}^{z}=0, \quad \ddot{q}^{X}+\Gamma_{Y Z}^{X} \dot{q}^{Y} \dot{q}^{Z}=0 .
$$

By taking the time derivative of $T$ and using the geodesic equations above we find that $T$ is conserved, $\frac{d}{d t} T=0$, so that we indeed have a consistent solution of the equations of motion.

In order to prove that the functions $\alpha(t), \beta(t)$ and $\nu(t)$ given by (6.2) are smooth, we only need to show that the piecewise constant function $T$ is continuous at the transition point. Looking at the definition (2.44), we find that $T$ contains the vector and hypermultiplet scalar metrics as well as $\dot{\phi}^{x}$ and $\dot{q}^{X}$. The metrics have already been shown to be continuous. To establish the continuity of the scalar fields, we observe that their dynamics is governed by the geodesic equations (6.4). These differential equations are different at both sides of the transition line because of the 
discontinuity in the Christoffel symbols $\gamma_{y z}^{x}$. But since eq. (6.4) is of second order, we can choose the constants of integration in such a way that the scalar fields $\phi^{x}, q^{X}$ as well as their first derivatives $\dot{\phi}^{x}, \dot{q}^{X}$ are continuous at the transition locus. Then $T$ is continuous at the transition line. This completes the proof of the statement given above.

Note that we only get a smooth solution if we choose a particular lapse function. This choice is distinguished by the fact that the gravitational and scalar equations of motion decouple. Moreover, the time variable corresponding to our lapse function is the affine parameter of the geodesic equations on the moduli spaces. When studying cosmological solutions, however, we will use the standard cosmological time $\tau$, which amounts to setting the lapse function to unity. In this parametrization the second derivatives of $\alpha(\tau)$ and $\beta(\tau)$ are continuous, but have a kink when crossing the transition locus. This frame dependence of solutions resembles the differences between the string frame and the Einstein frame familiar from string theory.

\subsection{Cosmological solutions of the $\mathbb{F}_{\mathbb{1}}$-model}

We now turn to the cosmological solutions of the $\mathbb{F}_{1}$-model. In order to have the standard parametrization used in cosmology we now switch to the cosmological time $\tau$ so that the space-time metric (2.39) becomes

$$
\mathrm{d} s^{2}=-\mathrm{d} \tau^{2}+\mathrm{e}^{2 \alpha(\tau)} \mathrm{d} \vec{x}^{2}+\mathrm{e}^{2 \beta(\tau)} \mathrm{d} y^{2}
$$

Here and henceforth it is understood that all fields and the functions $\alpha$ and $\beta$ now depend on $\tau$ and we use the "dot" to indicate derivatives with respect to $\tau$. The corresponding equations of motion can be obtained from (2.41), (2.42) and (2.43) by setting $\nu=\dot{\nu}=0$ and replacing $t \rightarrow \tau$. The vector multiplet scalar metric is given by eq. (C.7) where the choice of metric depends on in which region the solution evolves. Further we restrict the hypermultiplet sector to the universal hypermultiplet with 
metric (3.19). The scalar potential is set to zero, since the transition states have been integrated out.

Parametrizing the solutions in cosmological time, we note that the Einstein and matter equations do not decouple anymore. By taking certain linear combinations, Einstein equations may now be written as

$$
\begin{aligned}
3\left(\dot{\alpha}^{2}+\dot{\alpha} \dot{\beta}\right) & =T(\tau), \\
3\left(\ddot{\beta}+\dot{\beta}^{2}+2 \dot{\alpha} \dot{\beta}-\dot{\alpha}^{2}\right) & =-T(\tau), \\
3\left(\ddot{\alpha}+2 \dot{\alpha}^{2}\right) & =-T(\tau) .
\end{aligned}
$$

In this parametrization $T$ is no longer conserved along the integral curves of (2.42) and (2.43) and therefore depends on $\tau$.

\section{Possibilities for inflation}

In order to discuss whether this model allows inflation, we first introduce the scale factors

$$
\begin{array}{ll}
a=\mathrm{e}^{\alpha}, & \dot{a}=\dot{\alpha} \mathrm{e}^{\alpha}, \quad \ddot{a}=\left(\ddot{\alpha}+\dot{\alpha}^{2}\right) \mathrm{e}^{\alpha}, \\
b:=\mathrm{e}^{\beta}, & \dot{b}=\dot{\beta} \mathrm{e}^{\beta}, \quad \ddot{b}=\left(\ddot{\beta}+\dot{\beta}^{2}\right) \mathrm{e}^{\beta} .
\end{array}
$$

An expansion of space-time in the $\vec{x}$ - and $y$-dimensions is characterized by $\dot{a}>0$ and $\dot{b}>0$, while accelerated expansion corresponds to $\ddot{a}>0$ and $\ddot{b}>0$, respectively.

Rewriting Einstein's equations (6.6) in terms of $\ddot{a}$ and $\ddot{b}$ and taking appropriate linear combinations leads to

$$
\ddot{a}=-\left(\frac{1}{3} T+\dot{\alpha}^{2}\right) \mathrm{e}^{\alpha}, \quad \ddot{b}=\left(-T+3 \dot{\alpha}^{2}\right) \mathrm{e}^{\beta} .
$$

These equations show that the Out-picture does not allow for accelerated expansion in the $\vec{x}$-dimensions, since $\ddot{a}$ is negative semi-definite. An accelerating phase in the $y$-dimension is possible and requires $3 \dot{\alpha}^{2}>T$. 


\section{Numerical Examples}

We will now study numerical solutions of the eqs. (2.41), (2.42) and (2.43) parametrized by the cosmological time $\tau$. In this course we restrict the universal hypermultiplet sector of our model to the volume scalar $V$ and set $\sigma, \theta, \tau$ to zero which can be done consistently. In this case eq. (2.43) becomes

$$
\ddot{V}-\frac{1}{V} \dot{V}^{2}+(3 \dot{\alpha}+\dot{\beta}) \dot{V}=0
$$

We first focus on the dynamics of the vector multiplet scalar fields $U, W$. Their typical dynamics is shown in figure 1. Here the arrows point towards increasing values of $\tau$. But the equations of motion (2.41), (2.42) and (2.43) are invariant under time reversal $\tau \rightarrow-\tau$ so that each trajectory has a time-reversed counterpart.

The vector multiplet scalar trajectories may be classified according to the boundaries where they start and end. Figure 1 shows one example for every type of these trajectories. We find that for every possible pair of boundaries there is one class of trajectories which start at one of the boundaries and end at the other. ${ }^{29}$ All boundaries can be reached in a finite time which depends on the particular trajectory chosen. The solution "L" corresponds to a certain subclass of the types introduced above. Here the absolute value of the field derivatives decreases monotonically, so that the corresponding solution stays inside the vector multiplet moduli space for an exceptionally long time. None of the examples becomes singular while inside the extended Kähler cone. We further observe that all trajectories are one-time differentiable at the flop line $U=W$. Details on the behavior of the solutions at the other boundaries of the vector multiplet scalar manifold can be found in [FS4].

Let us now turn to the other fields appearing in the equations of motion. Examples of their characteristic behavior are illustrated in figure 2. The initial conditions

\footnotetext{
${ }^{29}$ For the $U=0$ boundary $b_{1}$ this is not obvious from figure 1 . The numerical analysis indicates that the trajectories which leave the plot at $W=3$ approach the boundary $b_{1}$ at large values of $W$. However, the numerical solutions are not conclusive about whether the boundary is reached at a finite of infinite value of $W$.
} 

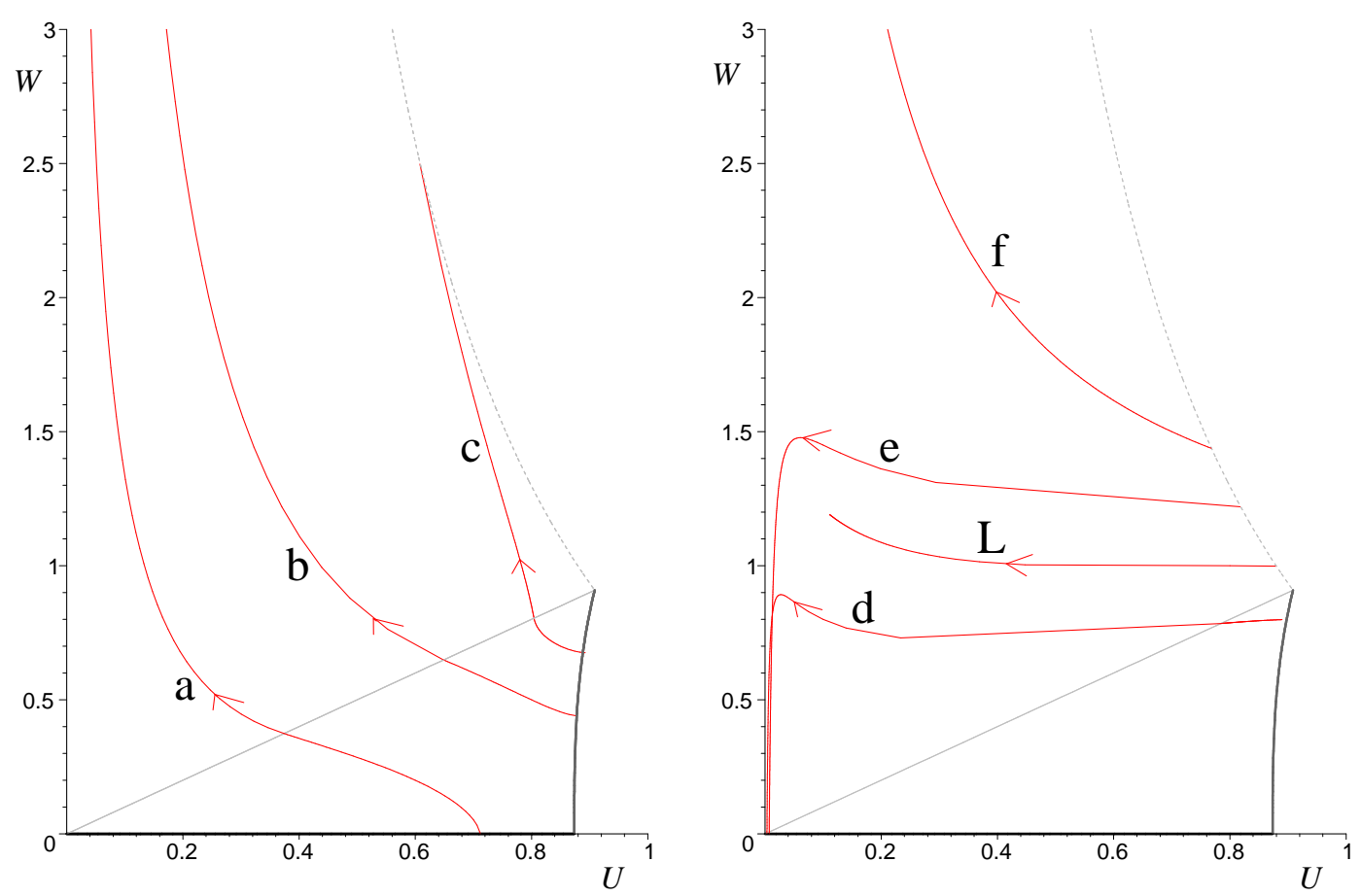

Figure 1: Illustrative examples for possible solutions of the Out-picture equations of motion with initial conditions given in table 2 in the appendix $\mathrm{C}$. The solutions " $\mathrm{a}$ " to " $\mathrm{f}$ " connect all possible boundaries of the extended Kähler cone. Solution "L" is an example for a solution that starts at the boundary and remains inside the moduli space for an exceptionally long time.

for these trajectories are given by the lines "b", "d" and "L" in table 2.

The first row of figure 2 shows the behavior of the vector multiplet scalar fields $U(\tau), W(\tau)$. The first two diagrams display the solutions "b" and "d" which both start at the boundary $b_{3}$ and end at $b_{1}$ and $b_{2}$, respectively. ${ }^{30}$ The second row shows the behavior of the CY volume $V$. Here we observe that $V(\tau)$ either increases or decreases monotonically, depending on whether the solution started with $\dot{V}(0)>0$ or $\dot{V}(0)<0$. Choosing $\dot{V}(0)=0$ leads to a constant volume. We find that $V$ does not become singular, $V=0$ or $V=\infty$, while the solution is inside the extended Kähler cone. The evolution of the scale factors $\alpha(\tau)$ and $\beta(\tau)$ is shown in the third row of figure 2. All solutions have $\ddot{a} \leq 0$. Their behavior is governed almost entirely by their initial conditions $\dot{\alpha}(0), \dot{\beta}(0)$. For positive (negative) initial values

\footnotetext{
${ }^{30}$ For the definition of these boundaries see appendix C.
} 
$\mathrm{b}$
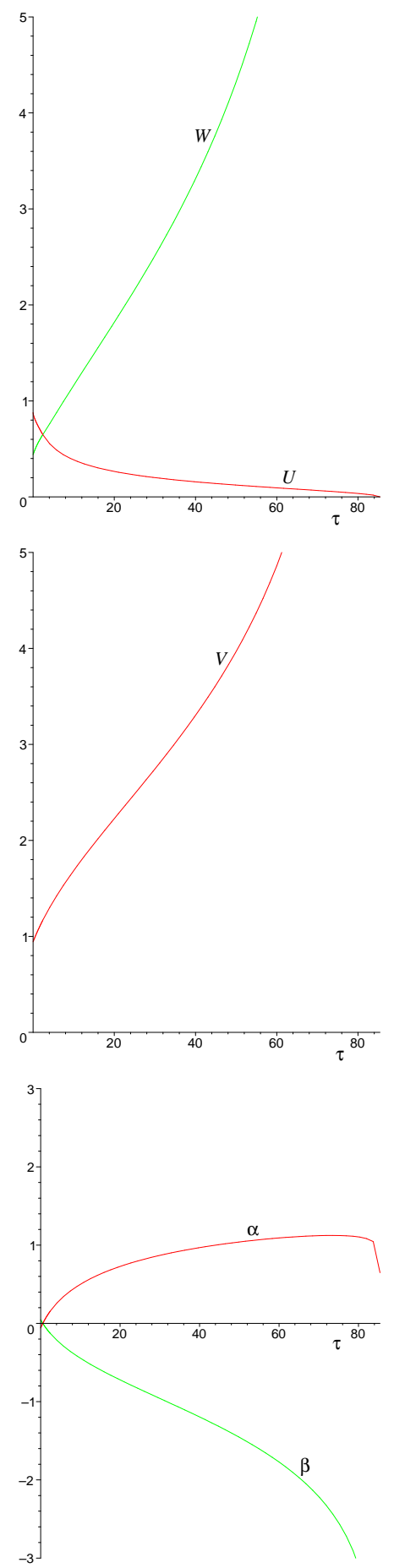

d
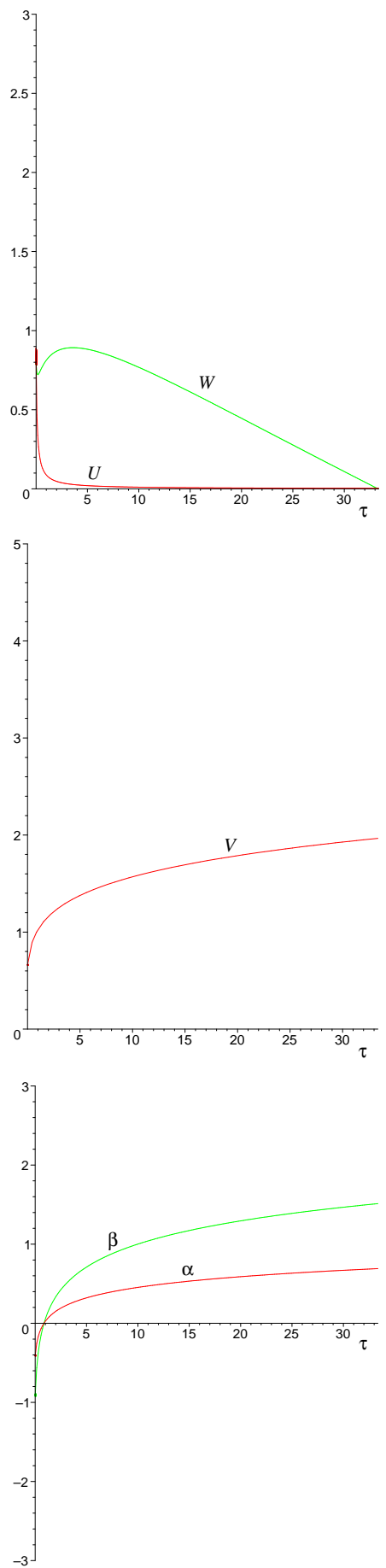

$\mathrm{L}$
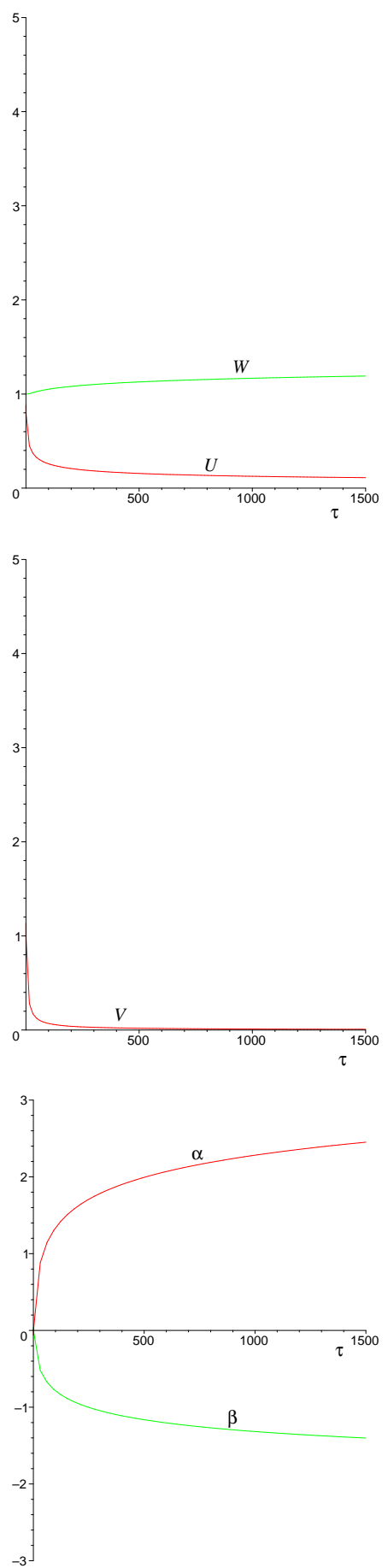

Figure 2: Three examples of solutions illustrating the typical behaviors of cosmological solutions in the Out-picture description of a flop transition. The solutions "b" and "d" show the usual run-away behavior of the moduli fields while the "L" solution stays inside the extended Kähler cone for an exceptionally long time. 
the solutions monotonically increase (decrease). The only exception to this rule arises when the solution approaches the boundary $b_{1}$. In this case the kinetic term $T$ becomes large due to the vector multiplet scalar metric $g_{x y}^{(\mathrm{III})}$ developing an infinite eigenvalue. This induces a rapid decrease of both $\alpha(\tau)$ and $\beta(\tau)$. But the solution only becomes singular at the boundary $b_{1}$. Therefore we do not encounter any spacetime singularities, while the vector multiplet scalars are inside the extended Kähler cone of the model. ${ }^{31}$

\section{Kasner cosmologies and In-picture flops}

After analyzing the behavior of cosmological solutions in the Out-picture description of a flop, we now turn to the corresponding In-picture. The new ingredient is the non-trivial scalar potential induced by the transition states. As we will show in the following, it is this new feature that can give rise to an accelerating phase in our cosmological solutions and leads to the dynamical stabilization of the moduli close to the flop. ${ }^{32}$ Thus we start the discussion of the In-picture by examining the properties of this potential.

\subsection{The scalar potential of the $\mathbb{F}_{1}$-model}

In subsection 4.1, we found that the potential arising in the In-picture description of the flop transition occurring in the $\mathbb{F}_{1}$-model is of the form (2.23). It is completely determined by the vector and hypermultiplet metrics obtained from (C.10) and (3.3) and the superpotential (4.7). The potential is positive semi-definite, $\mathbb{V}(\phi, q) \geq 0$. Its minima correspond to supersymmetric Minkowski vacua which are parametrized

\footnotetext{
${ }^{31}$ This is in agreement with the more general analysis [72, FS8].

${ }^{32}$ Since the In-picture LEEA is smooth, it is clear that Kasner solutions are smooth at flop transitions and also at any other transition involving finitely many transition states including conifold transitions.
} 

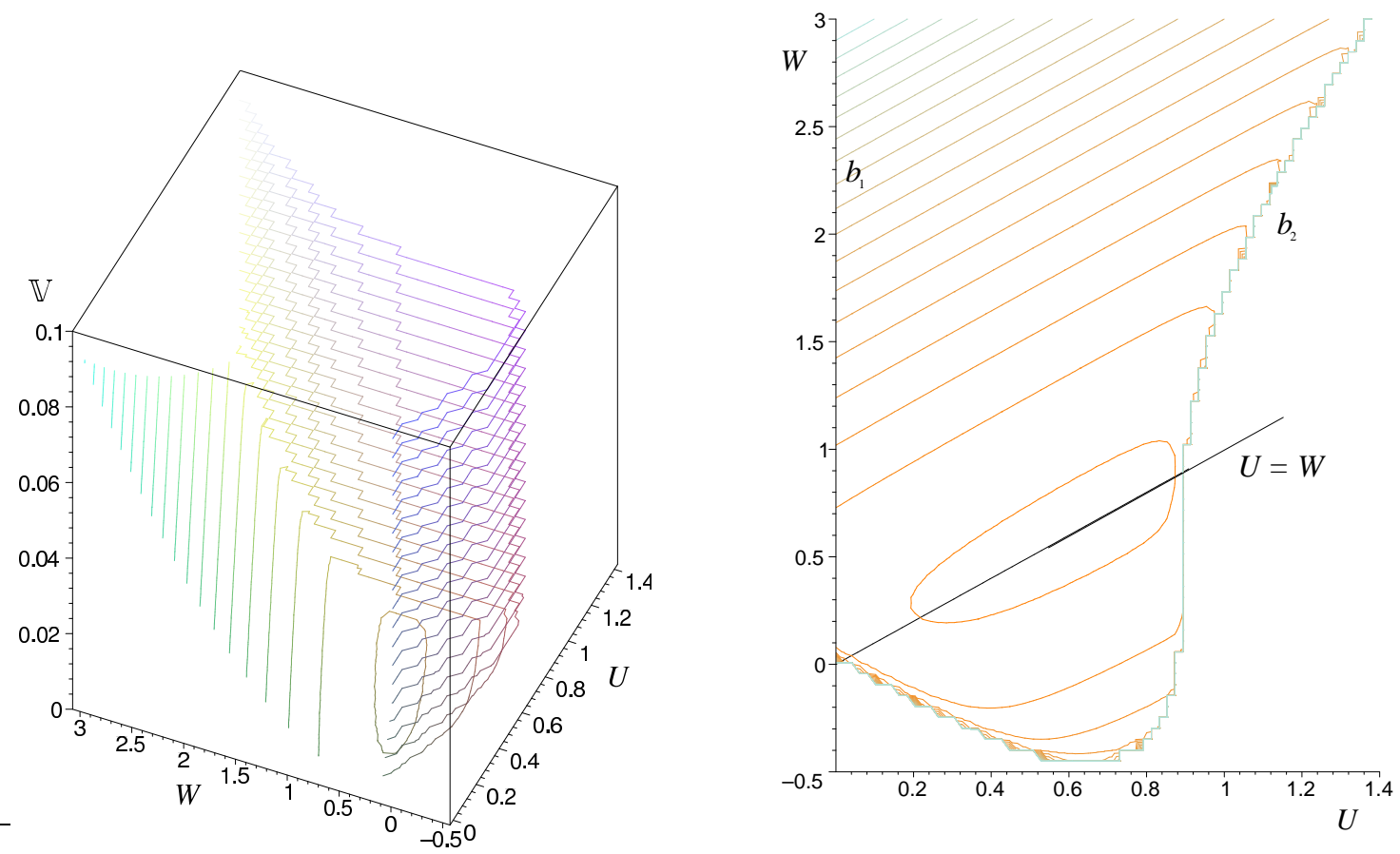

Figure 3: The potential $\mathbb{V}(\phi, q)$ at fixed values of the hypermultiplet scalars $u_{1}=u_{2}=0.2, v^{1}=$ $v^{2}=0$. The potential has its minimum in the vicinity of the flop line $U=W$. At the boundary ' $b_{2}$ ' where the metric $g_{x y}^{(\text {In) }}$ has a zero eigenvalue the potential diverges, while at the boundary ' $b_{1}$ ' where $g_{x y}^{(\mathrm{In})}$ becomes infinite it is finite.

by the subset $\mathcal{M}_{\text {Coul }}$ of the scalar manifold given in eq. (4.11). These minima are the only critical points of the potential.

Figure 3 shows the potential $\mathbb{V}(\phi, q)$ for some non-trivial but fixed values of the hypermultiplet scalar fields. The potential is positive definite and finite as long as we are inside the vector multiplet scalar manifold. While figure 3 clearly shows that the value of the potential is small in the vicinity of the flop line $U=W$, an explicit calculation reveals that its actual minimum for fixed non-zero values of the transition states is not located at the flop line but slightly next to it. ${ }^{33}$ The potential diverges at the boundary $b_{2}$ where the vector multiplet metric $g_{x y}^{(\text {In) }}$ has a zero eigenvalue. At the boundary $b_{1}$, where $\operatorname{det}\left(g_{x y}^{(\mathrm{In})}\right)$ is infinite, the potential is finite. This feature can

\footnotetext{
${ }^{33}$ Note that this point is not a critical point of the potential, since the derivatives with respect to the hypermultiplet scalars does not vanish.
} 
be traced back to the second term of the scalar potential (2.23) which contains the inverse metric $g^{(\mathrm{In}) x y}$. Finally we observe that in the limit $W \rightarrow \infty$ the potential diverges quadratically, $\mathbb{V} \propto W^{2}$.

After considering the properties of the potential at the boundaries of the vector multiplet scalar manifold, let us comment on the boundaries appearing in the hypermultiplet sector. These are given by the loci where the hypermultiplet metric derived from (3.3) has an infinite eigenvalue. This occurs for either $\phi_{+}$or $\phi_{-}$defined in (3.4) becoming zero. The potential diverges at all boundaries of the hypermultiplet moduli space.

Figure 4 illustrates the dependence of the potential on the transition states. Taking $v^{2}=p, u_{2}=q$ both real and $v^{1}=u_{1}=0$ and substituting this restriction into $\phi_{+}$and $\phi_{-}$, we obtain

$$
\phi_{+}=1-p^{2}, \quad \phi_{-}=1-q^{2}+p^{2} q^{2} .
$$

This indicates that $p$ is bounded and takes values $-1<p<1$, while $q$ is unbounded.

In summary we find that the potential diverges at all boundaries of the moduli space where either the vector or the hypermultiplet scalar metric develops a zero eigenvalue. At the boundaries where $\operatorname{det}\left(g_{x y}^{(\mathrm{In})}\right)$ becomes infinite, the potential is finite. These features can also be deduced from eq. (2.23). However, as explained before, it is not clear to which extent the In-picture LEEA at the boundaries really captures the microscopic M-theory physics.

\subsection{Fixed points of the equations of motion}

After discussing the properties of the $\mathbb{F}_{1}$-model scalar potential, we now turn to the In-picture equations of motion. Before investigating their properties numerically, let us first obtain some analytic results by studying the fixed point properties of 


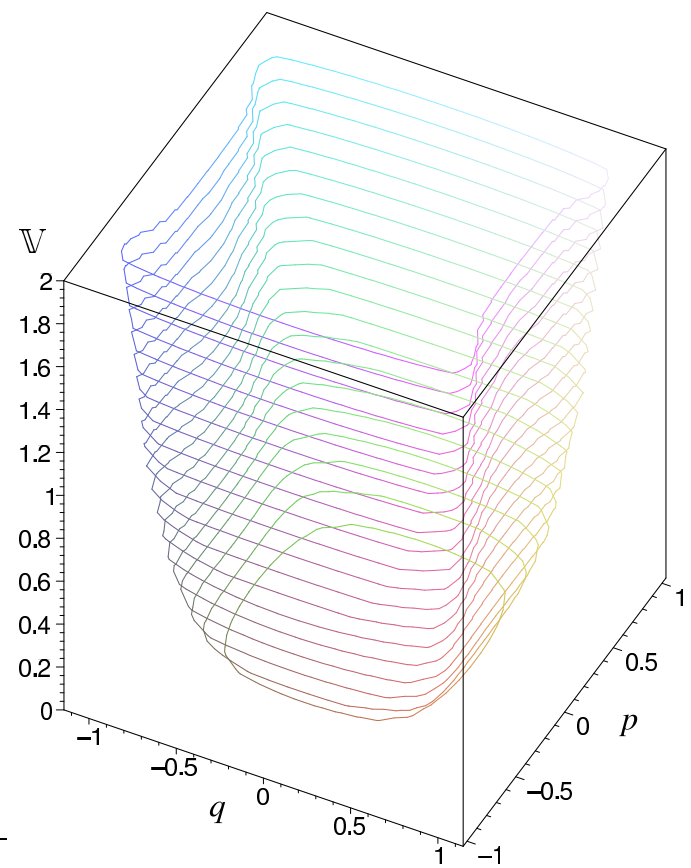

Figure 4: The scalar potential restricted to the submanifold $U=W=0.6, v^{1}=u_{1}=0$ and $v^{2}=p, u_{2}=q$ taken to be real. The potential diverges at the loci where $\phi_{+}$or $\phi_{-}$become zero.

these equations. We begin by rewriting the system of second order equations (2.41), (2.42) and (2.43) as a set of coupled autonomous first order equations. This is done in the standard way by introducing the momentum variables:

$$
p^{X}:=\dot{q}^{X}, \quad \rho^{x}:=\dot{\phi}^{x}, \quad \gamma:=\dot{\alpha}, \quad \delta:=\dot{\beta}
$$

Substituting in these variables, the equation of motion can be written as

$$
\dot{q}^{X}=\beta_{q}^{X}, \dot{p}^{X}:=\beta_{p}^{X}, \dot{\phi}^{x}=\beta_{\phi}^{x}, \dot{\rho}^{x}=\beta_{\rho}^{x}, \dot{\gamma}=\beta_{\gamma}, \dot{\delta}=\beta_{\delta}
$$

where the constraint arising in the Einstein equations takes the form

$$
\delta=\frac{1}{\gamma}\left(\frac{1}{3}\left(T+\mathrm{g}^{2} \mathbb{V}\right)-\gamma^{2}\right)
$$


In these expressions the functions $\beta_{i}$ are given by:

$$
\begin{aligned}
& \beta_{q}^{X}=p^{X}, \quad \beta_{\phi}^{x}=\rho^{x}, \\
& \beta_{p}^{X}=-(3 \gamma+\delta) p^{X}-\Gamma_{Y Z}^{X} p^{Y} p^{Z}-\mathrm{g}^{2} g^{X Y} \frac{\partial \mathbb{V}}{\partial q^{Y}}, \\
& \beta_{\rho}^{x}=-(3 \gamma+\delta) \rho^{x}-\gamma_{y z}^{x} \rho^{y} \rho^{z}-\mathrm{g}^{2} g^{x y} \frac{\partial \mathbb{V}}{\partial \phi^{y}}, \\
& \beta_{\gamma}=-\gamma \delta-3 \gamma^{2}+\frac{2}{3} \mathrm{~g}^{2} \mathbb{V}, \quad \beta_{\delta}=-3 \gamma \delta-\delta^{2}+\frac{2}{3} \mathrm{~g}^{2} \mathbb{V} .
\end{aligned}
$$

The set of fixed points of these equations consists of the points where the functions $\beta_{i}$ vanish simultaneously. For the matter fields the only solution to the equations $\beta_{q}^{X}=0, \beta_{\phi}^{x}=0$ is given by $p^{X}=0, \rho^{x}=0$. Substituting this constraint into $\beta_{p}^{X}, \beta_{\rho}^{x}$, we find that these functions vanish if $g^{\Lambda \Sigma} \frac{\partial \mathbb{V}}{\partial \phi^{\Sigma}}=0$. Since the metric $g^{\Lambda \Sigma}$ is non-degenerate, this condition requires $\frac{\partial \mathbb{V}}{\partial \phi^{\Sigma}}=0$. This is just the condition for a critical point of the potential, which has already been investigated in the previous subsection. Using the result (4.11) we see that the fixed points of the matter equations are

$$
\mathcal{M}_{C}^{\text {mat }}=\left\{\mathcal{M}_{\text {Coul }}, p^{X}=0, \rho^{x}=0\right\}
$$

Hence the fixed point manifold of the equations of motion is parametrized by the flat directions of the potential.

Concerning the Einstein equations, we first observe that under the condition (7.6) $T$ and $\mathbb{V}$ vanish identically. In this case $\beta_{\gamma}, \beta_{\delta}$ and the constraint (7.4) simplify to

$$
\left.\beta_{\gamma}\right|_{\mathcal{M}_{C}^{\text {mat }}}=-\gamma \delta-3 \gamma^{2},\left.\quad \beta_{\delta}\right|_{\mathcal{M}_{C}^{\text {mat }}}=-3 \gamma \delta-\delta^{2}, \quad \delta=-\gamma
$$

Applying the fixed point condition $\beta_{\gamma}=0, \beta_{\delta}=0$ implies that $\gamma=0, \delta=0$ while the values of $\alpha$ and $\beta$ are not determined by the fixed point condition. Thus we find 
that the equations (7.3) have an entire manifold of fixed points $\mathcal{M}_{C}^{\mathrm{FP}}$, given by

$$
\mathcal{M}_{C}^{\mathrm{FP}}=\left\{\begin{array}{l}
\mathcal{M}_{\mathrm{Coul}}, p^{X}=0, \rho^{x}=0, \gamma=0, \delta=0 \\
\alpha, \beta \text { undetermined. }
\end{array}\right.
$$

Let us now discuss the properties of these fixed points. To this end we calculate the critical exponents arising from linearizing the equations (7.3) in the vicinity of the fixed points. These exponents are given by the eigenvalues of the stability matrix

$$
\mathbf{B}_{i j}:=\left.\partial_{j} \beta_{i}\right|_{\mathcal{M}_{C}^{\mathrm{FP}}}, \quad\left\{i, j \in \alpha, \beta, \delta, \rho, \phi^{x}, \rho^{x}, q^{X}, p^{X}\right\}
$$

In order to calculate the entries of this matrix, we observe that

$$
\left.\frac{\partial \beta_{\phi}^{\Lambda}}{\partial \phi^{\Sigma}}\right|_{\mathcal{M}_{C}^{\mathrm{FP}}}=-\left.\mathrm{g}^{2} g^{\Lambda \Xi} \frac{\partial^{2} \mathrm{~V}}{\partial \phi^{\Sigma} \partial \phi^{\Xi}}\right|_{\mathcal{M}_{C}^{\mathrm{FP}}}
$$

is the negative of the mass matrix $\mathcal{M}_{\Sigma}^{\Lambda},(4.14)$. With this information at hand, it is now straightforward to compute the entries of $\mathbf{B}_{i j}$ with respect to the basis (7.9),

$$
\mathbf{B}_{i j}=\left[\begin{array}{cccccc}
0 & \mathbb{1}_{2} & 0 & 0 & 0 & 0 \\
0 & 0 & 0 & 0 & 0 & 0 \\
0 & 0 & 0 & \mathbb{1}_{2} & 0 & 0 \\
0 & 0 & 0 & 0 & 0 & 0 \\
0 & 0 & 0 & 0 & 0 & \mathbb{1}_{8} \\
0 & 0 & 0 & 0 & -\mathcal{M}_{X}^{Y} & 0
\end{array}\right] .
$$

The eigenvalues of this matrix are either zero or purely imaginary. Here it is useful to distinguish between the directions corresponding to the fields $\alpha, \beta, \gamma, \delta, \phi^{x}, \rho^{x}, v^{1}, u_{1}, \dot{v}^{1}, \dot{u}_{1}$ and the charged transition states $v^{2}, u_{2}, \dot{v}^{2}, \dot{u}_{2}$. The corresponding eigenvalues for 
these fields are given by

$$
\theta^{\text {neutral }}=0, \quad \theta^{\text {trans }}= \pm i \sqrt{\frac{3}{2}} 6^{-1 / 3} \mathrm{~g}(U-W)
$$

respectively. This result implies that the fixed plane is neutrally stable, i.e., the solutions are neither attracted nor repelled by the fixed points. In other words these fixed points are non-hyperbolic $[73,74]$. Since in this case it is not guaranteed that the stability matrix encodes the behavior of the full non-linear system, we rely on numerical solutions. As we will see in the next subsection, the transition states indeed oscillate around their fixed point value $v^{2}=u_{2}=0$. The frequency of these oscillations depends on the actual values of the vector multiplet scalar fields. This is the same qualitative behavior as indicated in eq. (7.12).

\subsection{Numerical solutions}

We now turn to the numerical solutions of the equations of motion (2.41), (2.42) and (2.43). In order to be able to work with a trivial vector field background, we restrict the theory to the case where the hypermultiplet scalar fields are real, i.e.,

$$
Q_{v}=\operatorname{Re}\left(v^{1}\right), \quad q_{v}=\operatorname{Re}\left(v^{2}\right), \quad Q_{u}=\operatorname{Re}\left(u_{1}\right), \quad q_{u}=\operatorname{Re}\left(u_{2}\right)
$$

As discussed in subsection 2.4, this restriction allows us to work with a trivial vector field background. Further it is straightforward to check that it provides a consistent truncation of the hypermultiplet equations of motion (2.43).

To illustrate some characteristic features, we have picked a few examples of solutions whose initial conditions are given in table 3 in the appendix E. The solution labeled ' $\mathrm{b}$ " has the same initial conditions as the corresponding solution ' $b$ ' in the Out-picture, the only difference being one dynamical transition state for which we choose a non-vanishing initial value. This feature allows us to compare 

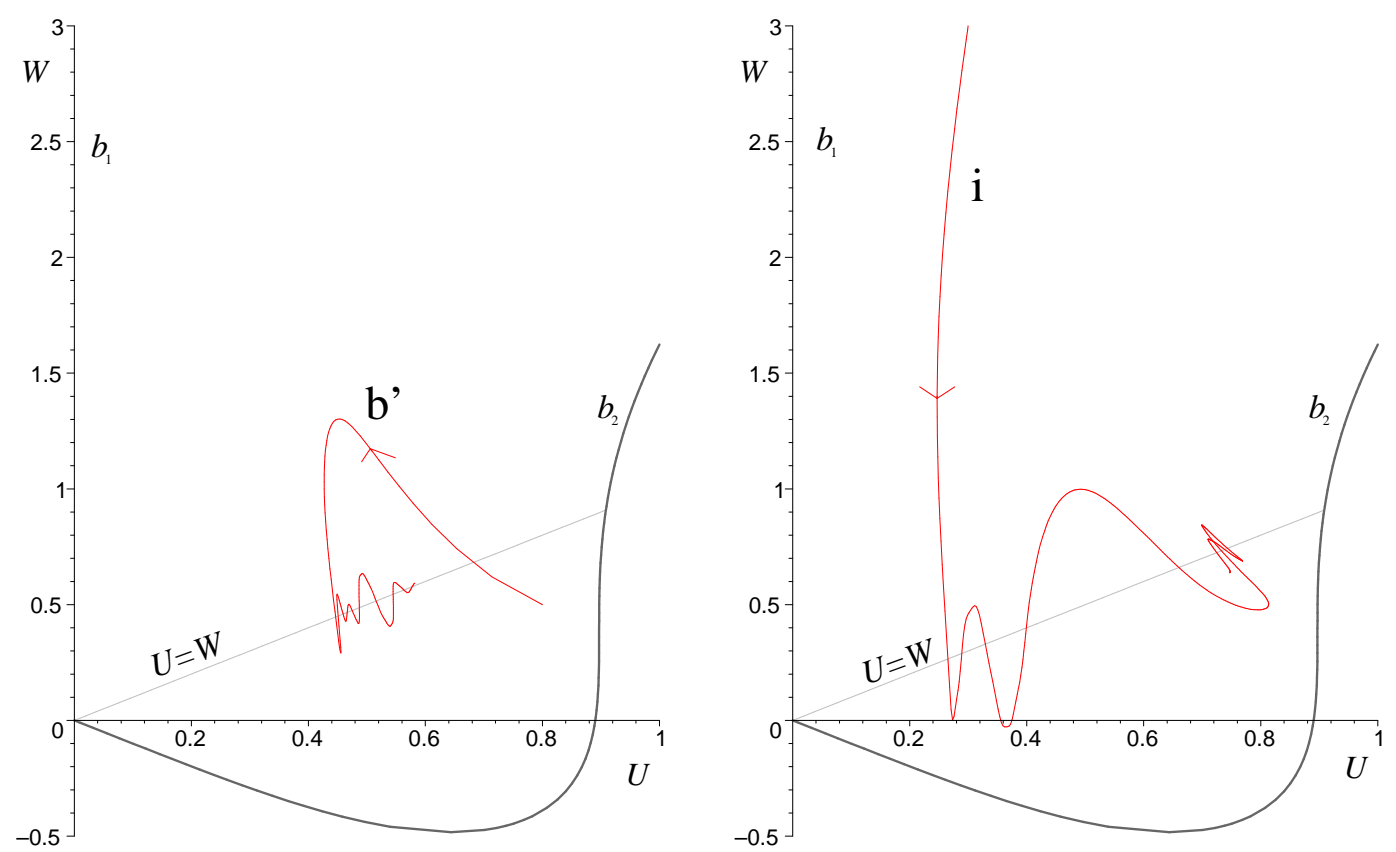

Figure 5: Numerical solutions of the In-picture equations of motion for the initial conditions ' $\mathrm{b}$ ', (left) and ' $\mathrm{i}$ ' (right) given in table 3. The trajectories $U(\tau), W(\tau)$ stabilize in the vicinity of the flop line. The right diagram also illustrates how the solution is repelled by the boundary $b_{2}$.

the qualitative behavior of the Out- and the In-picture solutions. Another example labeled ' $i$ ' illustrates the behavior of a solution which initially starts far away from the flop line.

Let us first focus on the dynamics of the vector multiplet scalars $U, W$. The trajectories of the example solutions ' $\mathrm{b}$ '" and ' $\mathrm{i}$ ' projected to the vector multiplet scalar manifold are shown in figure 5. The solutions first approach the flop line before starting to oscillate around $U=W$. However, the flop line is not an attractor: the solutions oscillate in the region close to the flop, but do not settle down at a fixed point. This generic behavior of solutions differs from the corresponding behavior in the Out-picture, where the solutions crossed the flop line and did not stabilize. The In-picture solution ' $\mathrm{b}$ ' ' initially evolves analogously to its Out-picture cousin ' $\mathrm{b}$ ' but after crossing the flop line is turned back by the potential. A similar effect is shown in the second diagram, where one can also observe that the trajectory gets 
repelled when approaching the boundary $b_{2}$. Hence the In-picture potential provides a mechanism that prevents the solutions from running into the boundaries where $\operatorname{det}\left(g_{x y}^{(\mathrm{In})}\right)=0$. This is different from the Out-picture, where such boundaries are reached in finite time.

Let us now focus on the solution ' $\mathrm{b}$ ' more closely. The corresponding moduli are shown in figure 6 . We observe that the vector multiplet moduli $U(\tau)$ and $W(\tau)$ oscillate around the flop line $U=W$ instead of approaching the boundary $b_{1}$. The CY volume $V(\tau)$ is obtained by substituting the numerical solution into eq. (3.20). We find that when picking the initial value $\dot{V}(0)=0.1$, as for the Out-picture solution 'b', the volume increases monotonically. This is completely analogous to the corresponding Out-picture solution. The difference between the two pictures is, however, that the Out-picture volume undergoes an accelerated increase while the volume in the In-picture shows decelerated increase.

The initial values for the transition states were chosen such that $q_{v}(\tau)$ is frozen to be zero. The non-trivial initial value for $q_{u}$ results in $q_{u}(\tau)$ oscillating around $q_{u}=0$. The frequency of oscillations depends on the difference $|U(\tau)-W(\tau)|$ in the sense that a large difference induces rapid oscillations while a small difference, i.e., being close to the flop, corresponds to a low frequency. After some initial period the logarithmic scale factors $\alpha(\tau)$ and $\beta(\tau)$ become almost constant. This differs from the Out-picture where $\alpha(\tau) \rightarrow-\infty, \beta(\tau) \rightarrow-\infty$ as the solution approaches the boundary $b_{1}$.

So, comparing these examples of In- and Out-picture solutions we find that the inclusion of the charged transition states drastically modifies the behavior of the solution. In particular, the In-picture solution does not run into a boundary where the solution becomes singular.

The complete solution of our second example ' $i$ ' is shown in figure 7. Here we have taken the vector multiplet scalar $W$ to start "far away" from the flop line and the initial conditions for $Q_{u}$ and $Q_{v}$ were chosen such that the CY volume stays constant, $V(\tau)=1$. 

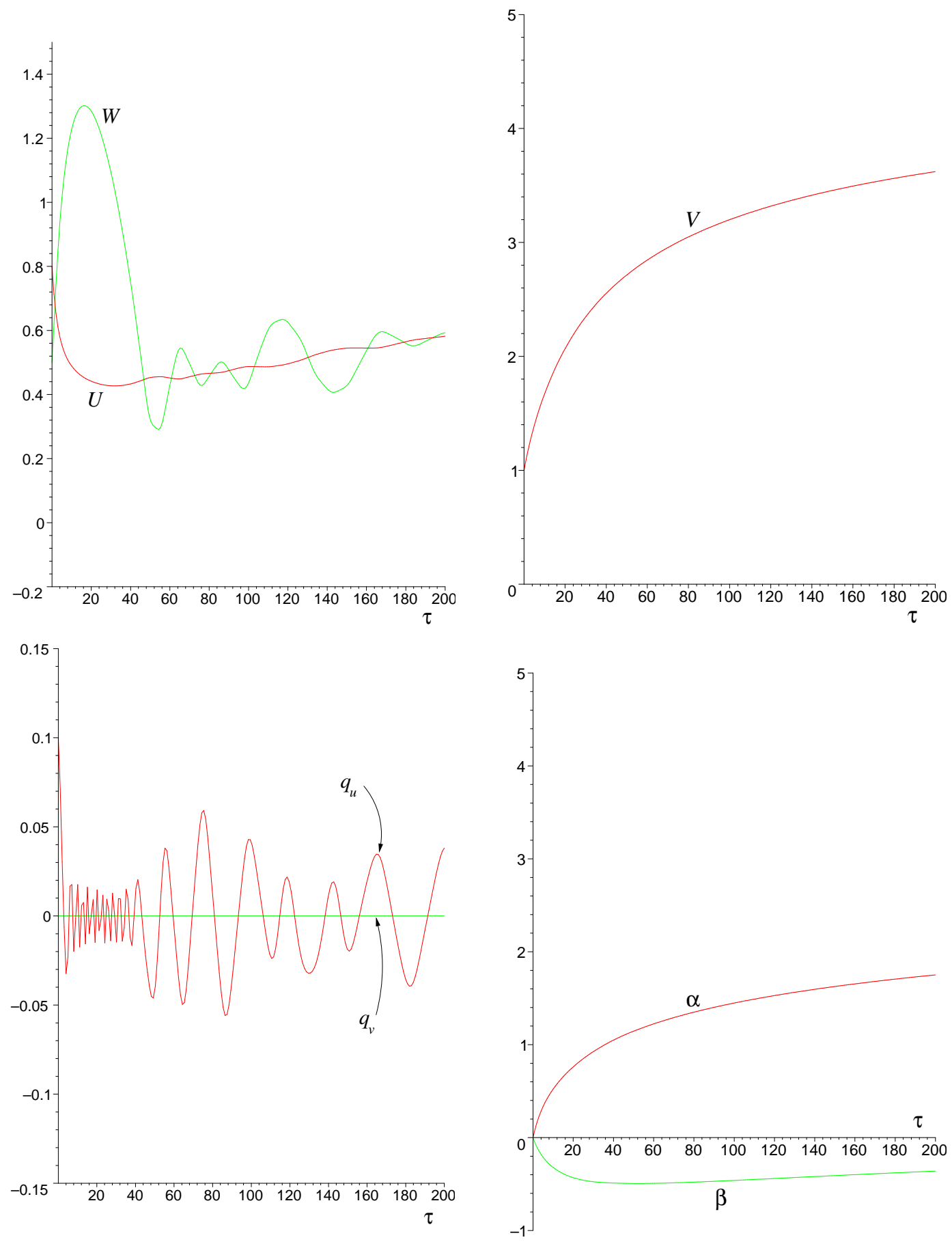

Figure 6: The complete numerical solution of eqs. (2.41), (2.42) and (2.43) starting with the initial values ' $\mathrm{b}$ ' given in table 3 . These are the same as for the Out-picture solution ' $\mathrm{b}$ ' except for the non-trivial transition state $q_{u}=0.1$. Starting from the upper left, the plots depict the dynamics of the vector multiplet scalars $U(\tau), W(\tau)$, the CY volume $V(\tau)$, the transition states $q_{u}(\tau), q_{v}(\tau)$ and the logarithmic scale factors $\alpha(\tau), \beta(\tau)$, respectively. After some initial period the CY volume stabilizes, the logarithmic scale factors increase monotonically, and $q_{u}(\tau)$ oscillates around the fixed plane value $q_{u}=0$. 

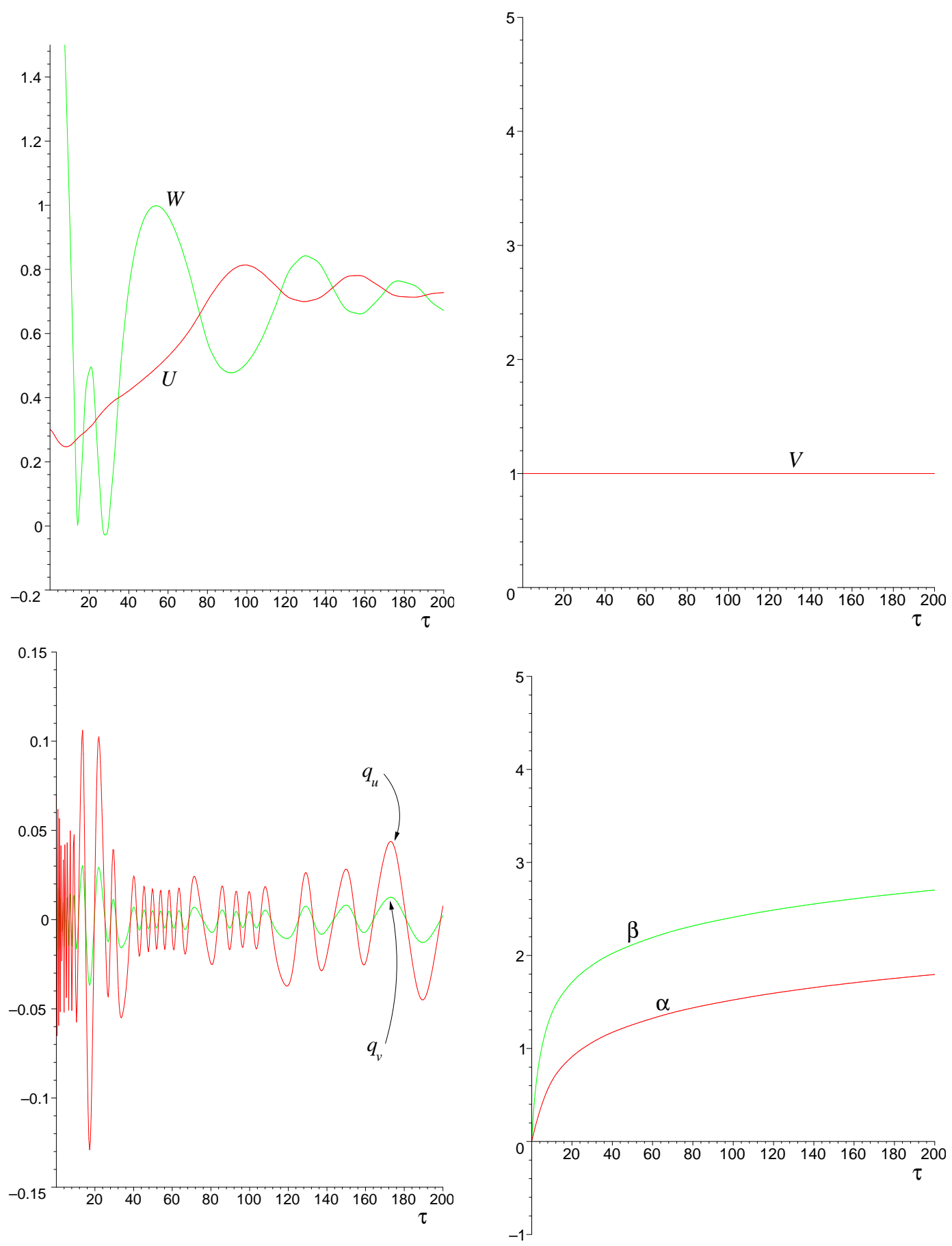

Figure 7: The complete numerical solution of eqs. (2.41), (2.42) and (2.43) starting with the initial values ' $\mathrm{i}$ ' given in table 3 . As in figure 6 , the diagrams show the dynamics of the vector multiplet scalars $U(\tau), W(\tau)$, the CY volume $V(\tau)$, the transition states $q_{u}(\tau), q_{v}(\tau)$ and the logarithmic scale factors $\alpha(\tau), \beta(\tau)$, respectively. The vector multiplet scalars are repelled when approaching the boundary $b_{2}$. The transition states $q_{u}(\tau), q_{v}(\tau)$ oscillate with exactly the same frequency. 

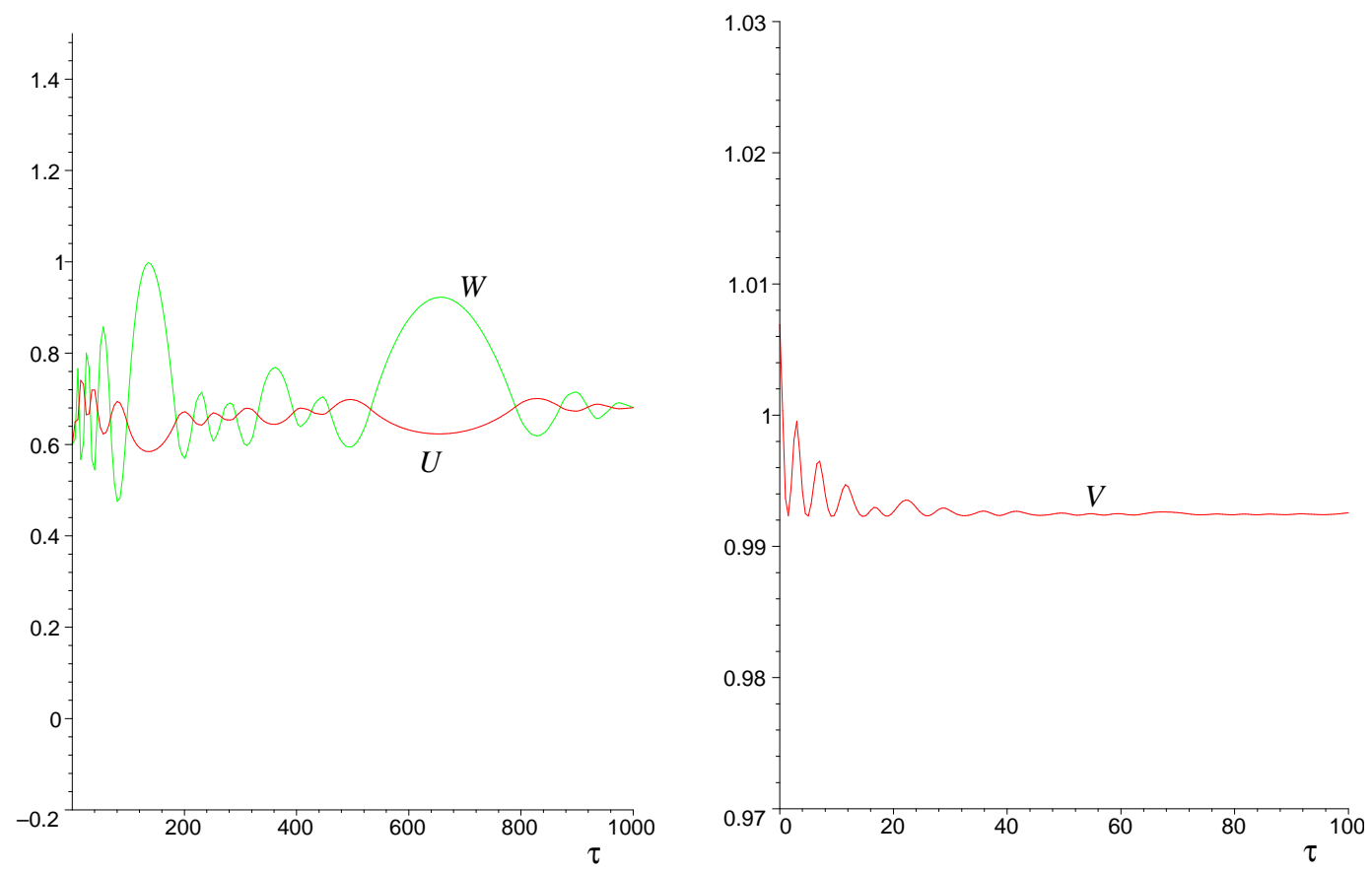

Figure 8: The left and right diagram show $U(\tau), W(\tau)$ and the CY volume $V(\tau)$ arising from the initial conditions ' $\mathrm{g}$ ' given in table 3 . The solution starts at the flop line but then evolves away and oscillates around $U=W$. Furthermore, $V(\tau)$ is no longer monotonic but oscillates before stabilizing.

The scalars $U(\tau), W(\tau)$ start in a region of high potential so that the solution first rolls down to the region of low potential before it begins to oscillate around the flop line. The first turning point in $W(\tau)$ and the second turning point in $U(\tau)$ correspond to points where the solution is repelled by the boundary $b_{2}$. In the region where $|U(\tau)-W(\tau)|$ is large the moduli $q_{u}(\tau)$ and $q_{v}(\tau)$ exhibit rapid oscillations around the zero line. The frequency of the oscillations is identical for both $q_{u}(\tau)$ and $q_{v}(\tau)$. The logarithmic scale factors $\alpha(\tau)$ and $\beta(\tau)$ increase monotonically with time. In fact, a closer look on their dynamics in subsection 7.4 reveals several short periods of accelerated expansion, although these do not significantly influence the behavior of $\alpha(\tau)$ and $\beta(\tau)$.

For the solution 'g' shown in figure 8 we have chosen our initial values (see table 3 ) for $U$ and $W$ to be on the flop line. However, the solution does not stay at the 
flop but starts oscillating around it. This result illustrates again that the flop line is neither a stable nor an unstable manifold. Another notable feature of this solution is that the CY volume $V(\tau)$ is no longer monotonic, but oscillates before settling to a finite value. This behavior is caused by giving non-trivial initial values to the transition states.

A common property of all the solutions presented in this subsection is that if we evolve them backwards in time, they become singular. Here the magnitude of $\dot{\alpha}(\tau)$ and $\dot{\beta}(\tau)$ becomes increasingly large, while the moduli $q^{X}, \phi^{x}$ are still inside their respective manifolds. As this happens, our numerical solutions are no longer reliable and we do not obtain any conclusive results about the origin of this singularity.

\subsection{The search for inflation}

In this subsection we investigate whether inflation is possible in the In-picture model. Equation (6.7) shows that the Out-picture does not admit accelerated expansion in the $\vec{x}$-directions, while in the $y$-direction the occurrence of acceleration depended on the actual value of $\dot{\alpha}$. However, contrary to the Out-picture, the In-picture contains a scalar potential, which, in principle, can give rise to an inflationary phase.

\section{General analysis of our model}

The starting point for our analysis are the Einstein equations (2.41) written in terms of the cosmological time $\tau$. Setting $\nu=0, \dot{\nu}=0$, these take the form:

$$
\begin{aligned}
3\left(\dot{\alpha}^{2}+\dot{\alpha} \dot{\beta}\right) & =T+\mathrm{g}^{2} \mathbb{V}, \\
2 \ddot{\alpha}+\ddot{\beta}+2 \dot{\alpha} \dot{\beta}+3 \dot{\alpha}^{2}+\dot{\beta}^{2} & =-T+\mathrm{g}^{2} \mathbb{V}, \\
3\left(\ddot{\alpha}+2 \dot{\alpha}^{2}\right) & =-T+\mathrm{g}^{2} \mathbb{V} .
\end{aligned}
$$

Eliminating the terms containing $\dot{\alpha} \dot{\beta}$ and rewriting the second and third equation in terms of $\ddot{a}$ and $\ddot{b}$ given in (6.7), we find the following analytic expressions for $\ddot{a}$ 
and $\ddot{b}$ :

$$
\ddot{a}=\left(\frac{1}{3}\left(\mathrm{~g}^{2} \mathbb{V}-T\right)-\dot{\alpha}^{2}\right) \mathrm{e}^{\alpha}, \quad \ddot{b}=\left(-\left(T+\frac{1}{3} \mathrm{~g}^{2} \mathbb{V}\right)+3 \dot{\alpha}^{2}\right) \mathrm{e}^{\beta} .
$$

These equations show that while $\mathbb{V}$ opposes accelerated expansion in the extradimension, it also enables accelerated expansion in the $\vec{x}$-directions. Acceleration in the three-space occurs if the potential dominates over the kinetic term $T$ and the $\dot{\alpha}$-contribution. In principle, eq. (7.15) allows for de Sitter like solutions, which correspond to $\mathbb{V}>0, \dot{\alpha}$ positive and approximately constant, and $T$ small compared to $\mathbb{V}$. However, since the ground state of our model is Minkowski, we cannot realize these de Sitter like solutions. The best approximation we can obtain are solutions which behave de Sitter like for a limited period of time. This can be realized when the scalar fields roll slowly at a non-vanishing value of the potential.

\section{Slow-Roll conditions}

Having found that our model admits inflationary phases in principle, we investigate whether there is a region in our parameter space where our potential satisfies the slow-roll conditions.

We consider the consistency conditions given in terms of the slow-roll parameter $\epsilon$ [49], generalized to non-linear sigma models (see for example [75]). Since the existence of a slow-roll regime is a property of the scalar potential $\mathbb{V}(\phi, q)$, we will drop the $(3+1)$-split of our space-like directions and set $\alpha(\tau)=\beta(\tau)$. We also introduce the standard Hubble parameter $H=\dot{\alpha}$. In terms of the covariant derivative with respect to the scalar field metrics, $D_{\tau} \dot{\phi}^{\Lambda}=\ddot{\phi}^{\Lambda}+\Gamma_{\Sigma \Xi}^{\Lambda} \dot{\phi}^{\Sigma} \dot{\phi}^{\Xi}$, the equations of motion (2.41), (2.42), and (2.43) imply

$$
H^{2}=\frac{1}{6}\left(T+\mathrm{g}^{2} \mathbb{V}\right), \quad D_{\tau} \dot{\phi}^{\Sigma}+4 H \dot{\phi}^{\Sigma}=-\mathrm{g}^{2} g^{\Sigma \Lambda} \frac{\partial \mathbb{V}}{\partial \phi^{\Lambda}} .
$$

Here $\left(\phi^{\Sigma}\right)=\left(\phi^{x}, q^{X}\right)$ and $\Gamma_{\Sigma \Xi}^{\Lambda}$ is the Christoffel connection with respect to the 
combined scalar metric $g_{\Lambda \Xi}=g_{x y} \oplus g_{X Y}$. Slow-roll inflation implies the following conditions:

$$
T \ll g^{2} \mathbb{V}, \quad D_{\tau} \dot{\phi}^{\Sigma} \ll 4 H \dot{\phi}^{\Sigma}
$$

In the slow-roll regime we can use the eq. (7.16) to express $\epsilon:=\frac{T}{g^{2} \mathbb{V}}$ entirely in terms of the potential,

$$
\epsilon \approx \frac{3}{16 \mathbb{V}^{2}} g^{\Sigma \Lambda} \frac{\partial \mathbb{V}}{\partial \phi^{\Sigma}} \frac{\partial \mathbb{V}}{\partial \phi^{\Lambda}}
$$

Consistency of the slow-roll conditions requires $\epsilon \ll 1$. By substituting $\alpha(\tau)=\beta(\tau)$ into eq. (2.40) and eliminating $\dot{\alpha}(\tau)$, we find that an accelerated expansion needs $3 T<\mathrm{g}^{2} \mathbb{V}$. Hence the period of inflation ends when $\epsilon=\frac{1}{3}$.

We then use this generalized slow-roll parameter $\epsilon$ (7.18) to investigate the possibility of slow-roll inflation for the In-picture scalar potential $\mathbb{V}(\phi, q)$. An extended numerical check shows that the condition for slow-roll inflation, i.e., the right hand side of eq. (7.18) being less than one third, is never satisfied. Thus we conclude that our scalar potential does not allow for a phase of slow-roll inflation.

\section{Numerical examples}

Even though our model does not admit slow-roll inflation, it nevertheless has solutions with short periods of accelerated expansion. This is not in conflict with the results above, as the conditions leading to (7.18) may be violated so that the approximation which we used to show that $\epsilon$ is bigger than one third breaks down.

Let us return to the numerical solutions ' $\mathrm{b}$ ' and ' $\mathrm{i}$ ' studied in subsection 7.3. The functions $\ddot{a}(\tau)$ and $\ddot{b}(\tau)$ for these solutions are shown in the left and right diagram of figure 9 , respectively. In both cases $\ddot{a}(\tau)$ and $\ddot{b}(\tau)$ show an oscillatory behavior. But the most prominent feature is that both solutions have short periods of acceleration. Looking at the solution ' $\mathrm{b}$ ' we see that $\ddot{b}(\tau)$ is positive for an extended period. This corresponds to a decelerated contraction of the $y$-direction which later turns into expansion (see figure 6 ). In the three-dimensional $\vec{x}$-space we observe several short 

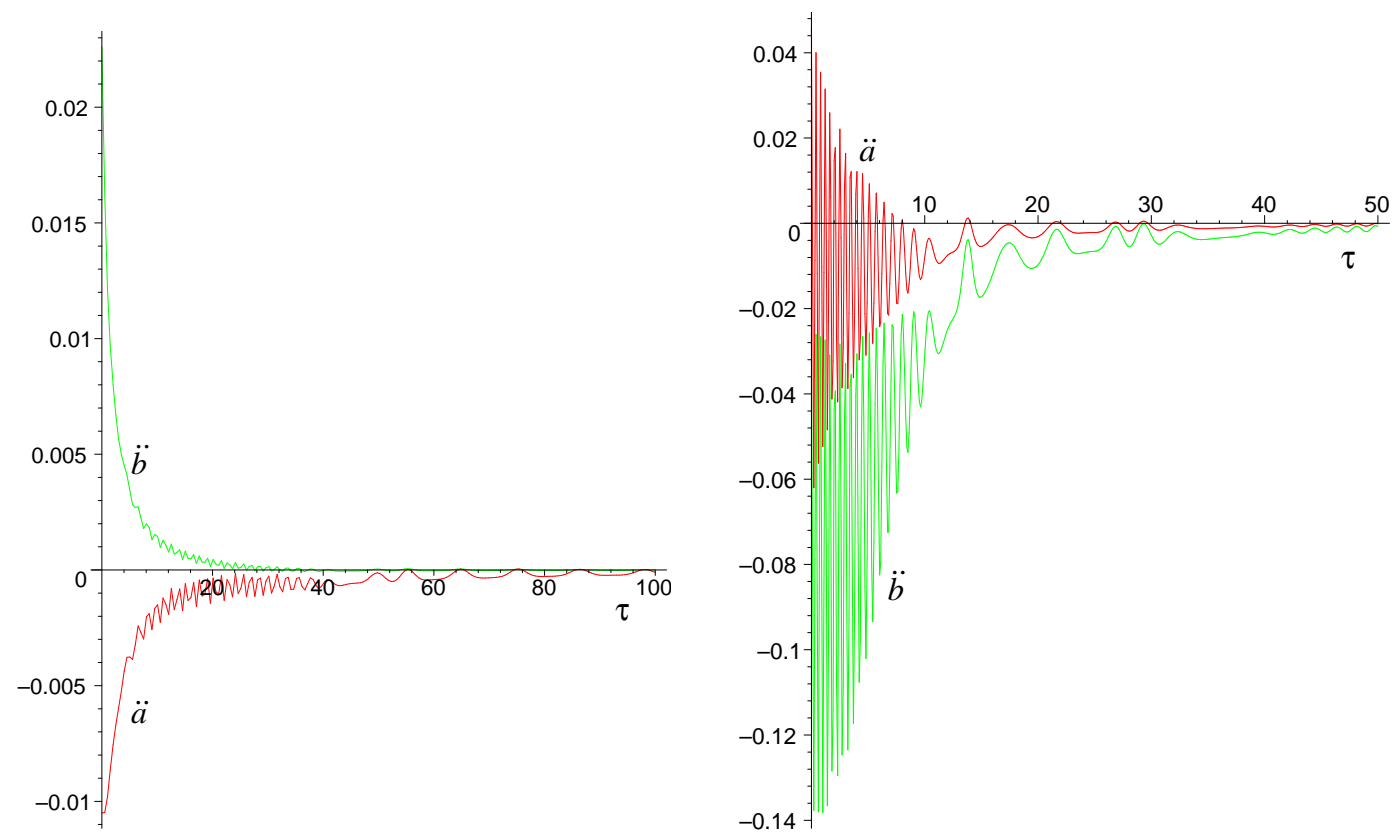

Figure 9: The factors $\ddot{a}(\tau)$ and $\ddot{b}(\tau)$ along the solutions ' $\mathrm{b}$ ' ' and ' $\mathrm{i}$ ' given in table 3 . Both solutions yield multiple short periods of accelerated expansion in three-space. The late time maxima are slightly positive.

periods of accelerated expansion at late times. For the solution 'i' these periods are more pronounced. Here $\ddot{b}(\tau)$ is initially negative and oscillates strongly whereas at later times we see small fluctuations. A common feature of the scale factors of both solutions is that after an initial period the average value of acceleration is small and negative, showing preference for a decelerating universe.

This behavior can also be understood from eq. (7.15). From there it is obvious that accelerated expansion in the three-space requires $\mathbb{V}(\phi(\tau), q(\tau))$ to dominate over $T(\phi(\tau), q(\tau))$ and $\dot{\alpha}(\tau)^{2}$ for some $\tau$. For our potential these requirements are most easily met if the transition states are non-zero and the vector multiplet moduli take values far away from the flop line. But there the transition states oscillate rapidly, and each time they pass through zero the potential vanishes, killing inflation.

These effects are also displayed by our numerical solutions. Looking at figure 6 shows that solution ' $\mathrm{b}$ " initially has a large value of $\dot{\alpha}(\tau)$, which for early times suppresses acceleration in $a(\tau)$ and induces acceleration in $b(\tau)$. For later times 
$\dot{\alpha}(\tau)$ decreases and the value of $\ddot{a}(\tau)$ is controlled by $T$ and $\mathbb{V}$. During this phase we observe decelerated expansion when $T$ dominates, while accelerated expansion corresponds to $\mathbb{V}$ dominating. The fluctuations in $\ddot{a}(\tau)$ reflect the fluctuations of the transition states. The potential vanishes if these are zero, so that at the corresponding points we automatically obtain decelerated expansion. On the other hand, if the numerical values of the transition states are large, the value of $\mathbb{V}$ also becomes large. These points are the most likely ones for $\mathbb{V}$ dominating over $T$ and give rise to the observed short phases of accelerated expansion. For the solution 'i' the effect of accelerated expansion is enhanced by choosing a small initial value of $\dot{\alpha}$ and $|U-W|$ being large, leading to large values of the potential and hence of $\ddot{a}$. The fluctuations in $\ddot{a}(\tau)$ are induced by the oscillations of the transition states, which cause oscillations in the value of the potential.

Note that the mechanism which leads to accelerated expansion in the above examples is rather generic. Acceleration is maximal at a collective turning point, where $T$ momentarily vanishes and the moduli fields turn from running "uphill" the potential to running "downhill" the potential. ${ }^{34}$ The farer the system is away from this point, the smaller is the resulting acceleration. As we have seen, the phases of acceleration in our model are not strong enough to induce an inflationary growth of the scale factors $a, b$. This is consistent with our earlier result that slow-roll inflation cannot be realized.

\footnotetext{
${ }^{34}$ It was pointed out in [76], that essentially the same mechanism is responsible for transient accelerating phases in cosmologies of hyperbolic and flux (S-brane) compactifications of string and M-theory.
} 


\section{Kasner cosmologies and conifold transitions}

After studying cosmological solutions of the $\mathbb{F}_{1}$-model we now return to the Inpicture description of the conifold transition. The aim of this section is to show that these transitions can occur dynamically. To this end we study Kasner type cosmological solutions of the minimal Lagrangian constructed in subsection 5.1. For explicitness and in order to be able to obtain numerical solutions we fix its vector multiplet sector by setting $c=0$ and use the M-theory value (4.16) for the gauge coupling g.

\subsection{The scalar potential of the minimal Lagrangian}

Before investigating the dynamics of the theory, let us discuss its scalar potential. In subsection 5.1 we found that it is of the form (2.23) with $\mathcal{W}$ given in (5.3). The potential is positive semi-definite, $\mathbb{V}(\phi, q) \geq 0$, and has two branches of supersymmetric Minkowski vacua given by $\mathcal{M}_{\text {Coul }}$ and $\mathcal{M}_{\text {Higgs }}$ in eq. (5.4) and (5.5), respectively.

To illustrate the structure of $\mathbb{V}(\phi, q)$ we restrict the hypermultiplet scalars to the one parameter subspace

$$
q:=\operatorname{Re}\left(u_{1}\right)=\operatorname{Re}\left(u_{2}\right), v^{1}=v^{2}=\operatorname{Im}\left(u_{1}\right)=\operatorname{Im}\left(u_{2}\right)=0 .
$$

On this subspace the Coulomb and the Higgs branch are given by $\phi \neq 0, q=0$ and $\phi=0, q \neq 0$, respectively. Further $\mathbb{V}(\phi, q)$ simplifies to

$$
\mathbb{V}(\phi, q)=\frac{3}{2\left(1-2 q^{2}\right)\left(1-\frac{3}{2} \phi^{2}\right)^{2 / 3}} q^{2} \phi^{2}
$$

This indicates that for $q \neq 0, \phi \neq 0$ the scalar potential diverges at the boundaries of the scalar manifolds given by $\phi_{ \pm}^{\text {crit }}= \pm \sqrt{\frac{3}{2}}$ and $q_{ \pm}^{\text {crit }}= \pm \sqrt{\frac{1}{2}}$. Even though these 


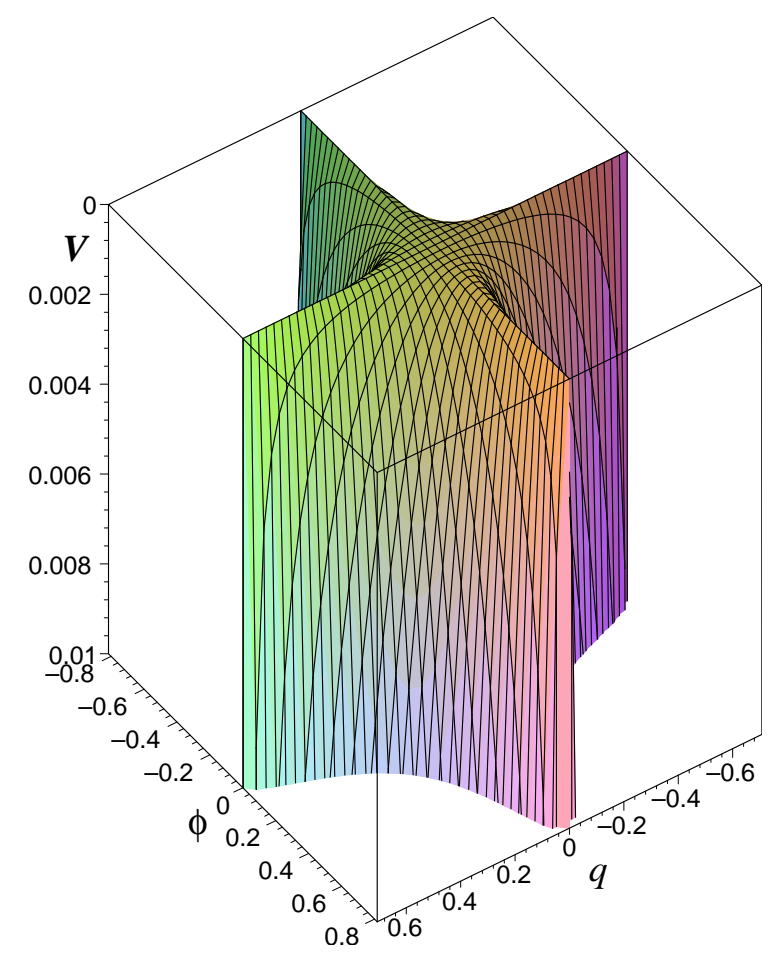

Figure 10: The scalar potential (8.2) of the minimal Lagrangian. The potential is positive semidefinite, but displayed 'upside down' in order to show the structure of the Coulomb- and the Higgs branch which manifests itself as a cross.

boundaries are located at finite coordinate distance, we point out that they have infinite geodesic distance from the origin.

The potential (8.2) is shown in figure 10. To make its vacuum structure manifest it is displayed 'upside down'. The Coulomb and the Higgs branch meet at the conifold locus $\phi=0, q=0$. Around this locus we have a central region where the potential almost vanishes. This central region and the vacuum branches are bounded by steep hyperbolic potential walls. We also observe that in the central region there is no energy barrier which obstructs that a classical solution evolves from the Coulomb to the Higgs branch and vice versa. 


\subsection{Conifold transitions of cosmological solutions}

We now turn to cosmological solutions of the minimal model. ${ }^{35}$ The corresponding equations of motion are obtained by substituting the vector and hypermultiplet scalar metrics (A.7) and (3.17) together with the scalar potential obtained from (5.3) into the eqs. (2.41), (2.42) and (2.43). To parametrize the solutions in cosmological time $\tau$ we also set $\nu=\dot{\nu}=0$. The resulting equations are further simplified by restricting them to the subspace (8.1) which allows us to work with a trivial vector field background and provides a consistent truncation of the equations of motion.

When solving these equations numerically, we are in particular interested in solutions which start on one vacuum branch and at some later time evolve along the other one. According to the discussion in subsection 5.1 such a solution has then undergone a conifold transition.

One example of such a solution is given in the figures 11 and 12 . The initial conditions of the trajectory are given in table 4 in the appendix E. Figure 11 displays the evolution of the scalar fields $\phi(\tau)$ and $q(\tau)$ and the logarithmic scale factors $\alpha(\tau)$ and $\beta(\tau)$ in the left and right diagram, respectively. The evolution of $\alpha(\tau)$ and $\beta(\tau)$ is completely analogous to the behavior found for the solution ' $\mathrm{b}^{\prime}$ ' displayed in figure 6 . The interesting new feature of this solution is the dynamics of the scalar fields. For $\tau \lesssim 250$, say, the absolute value of $q(\tau)$ is large while the one for $\phi(\tau)$ is very small, indicating that the solution evolves along the Higgs branch. For $250 \lesssim \tau \lesssim 350$ we encounter a crossover, where $|q(\tau)|$ and $|\phi(\tau)|$ are comparable and the solution evolves in the central region of the scalar potential. For $\tau \gtrsim 350$ the role of $q(\tau)$ and $\phi(\tau)$ have reversed. Now $q(\tau)$ has a very small modulus while $|\phi(\tau)|$ has become large. This indicates that the solution now evolves along the Coulomb branch, revealing that it has undergone a transition from one vacuum branch to the

\footnotetext{
${ }^{35}$ Note that this Lagrangian does not contain neutral hypermultiplets which, for CY compactifications, could play the role of the universal hypermultiplet. This can be interpreted as a truncation where all neutral hypermultiplets have been set to zero which, by virtue of eq. (3.20) corresponds to frozen $\mathrm{CY}$ volume, $V=1$.
} 

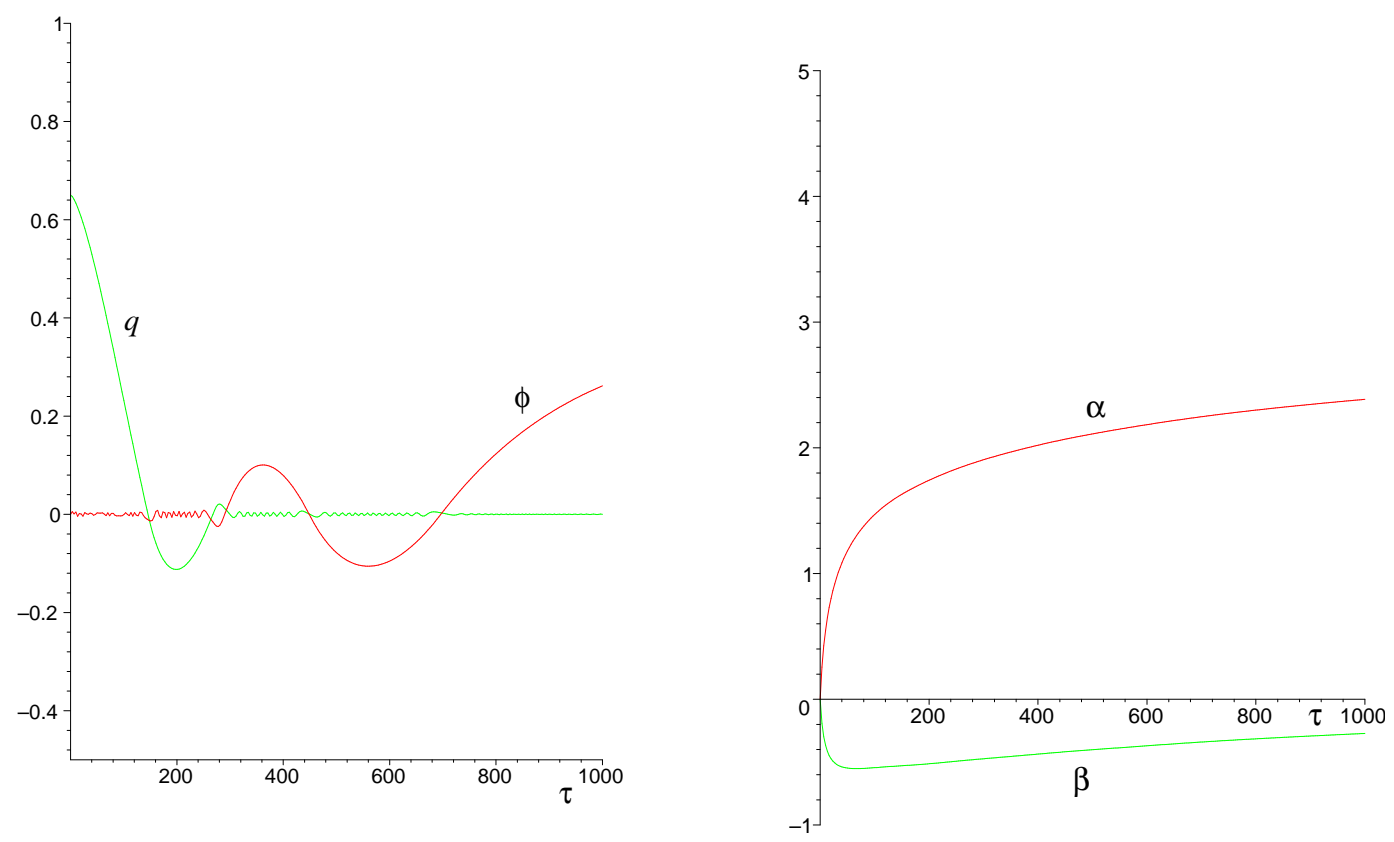

Figure 11: The scalars and logarithmic scale factors along a Kasner cosmological solution undergoing a conifold transition. The corresponding initial conditions are given in table 4 in the appendix E.

other.

Figure 12 shows the same solution projected onto the $\phi$-q-plane. Here the black hyperbolic lines correspond to equipotential lines of the scalar potential. The Coulomb and the Higgs branch equal the vertical $q=0$ and the horizontal $\phi=0$ axes, respectively. A conifold transition corresponds to the solution 'bending around the corner' when crossing from one vacuum branch to the other. For this bending to occur, the solution has to start with non-trivial initial values in both $\phi$ or $\dot{\phi}$ and $q$ or $\dot{q}$ as both $\phi=\dot{\phi}=0$ and $q=\dot{q}=0$ are consistent truncations of the equations of motion. This indicates that a solution starting with initial condition $\phi_{\text {init }}=\dot{\phi}_{\text {init }}=0$ always stays on the Higgs branch. Likewise $q_{\text {init }}=\dot{q}_{\text {init }}=0$ leads to a solution trapped to the Coulomb branch. Note that this implies that conifold transitions cannot occur dynamically in the Out-picture, since $q_{\text {init }}=\dot{q}_{\text {init }}=0$ is always satisfied in this case.

The second remarkable feature illustrated by figure 12 is that solutions evolving 


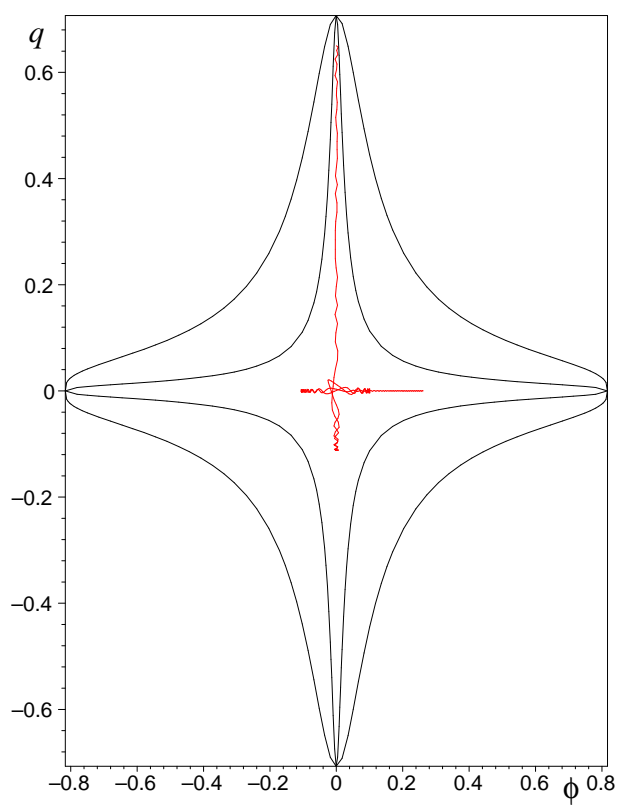

Figure 12: A cosmological solution undergoing a dynamical conifold transition projected to the $q$ - $\phi$-plane. The black lines are equipotential lines of the scalar potential. The four corners of the cross correspond to the boundaries of the scalar manifolds which are at infinite geodesic distance from the origin.

away from the central region experience an effective repulsive force which drives them back to the center. This in demonstrated by the turning points appearing if the solution "runs up" the Higgs or the Coulomb branch. ${ }^{36}$ Hence we find that, as in the case of flop transitions, the transition region is dynamically preferred even though the solution does not stabilize at the transition locus.

We further remark that there is some evidence that the equations of motion for the conifold are chaotic. Here we observe that evolving two solutions with almost identical initial conditions may result in the two solutions evolving at two different vacuum branches at some later point in time. This is similar to observations made for the potential $\mathbb{V}=x^{2} y^{2}$ which is also believed to exhibit chaotic behavior.

\footnotetext{
${ }^{36}$ For the potential $\mathbb{V}=x^{2} y^{2}$ this type of behavior was analyzed in [77] where it was found that there is an effective potential $\mathbb{V}_{\text {eff }} \propto \ln (x)$ driving the solutions back to the central region.
} 


\section{Summary and Conclusions}

In this thesis we constructed five-dimensional gauged supergravity actions which have all the essential features to describe flop and conifold transitions in M-theory compactified on Calabi-Yau threefolds. The new feature of these actions is that they explicitly include the extra light modes occurring in the transition region. The masses of these modes are encoded in the scalar potential. These actions smoothly connect the Calabi-Yau compactifications on the manifolds related by the transition, and there is no discontinuity or singularity arising at the transition locus. This is especially remarkable for conifold transitions, as these link Calabi-Yau compactifications with different Hodge numbers and therefore a different number of massless vector and hypermultiplets.

While the vector multiplet sector of these actions was determined exactly, we used a toy model based on the Wolf spaces $X(1+N)=\frac{U(1+N, 2)}{U(1+N) \times U(2)}$ to describe the hypermultiplets. In this context we worked out the metrics, the Killing vectors, and the moment maps of these spaces making use of the superconformal quotient construction $[40,41,42]$. This geometrical data suffices to determine any hypermultiplet sector based on $X(1+N)$ occurring in $\mathcal{N}=2$ supergravity in dimensions $3 \leq D \leq 6$. Furthermore, this approach considerably simplifies the investigation of gaugings, as the Killing vectors and moment maps are directly given in terms of the generators of the isometry group of the underlying Wolf space.

Our low energy effective actions have all the properties required to model flop and conifold transitions. Only the transition states acquire a mass away from the transition locus and the potential has a family of degenerate supersymmetric Minkowski ground states, which is parametrized by the moduli of $X$. None of the flat directions is lifted, and the number of vacuum branches exactly agrees with the requirements of the microscopic theory. Note that this is not implied by the charge assignment alone. The scalar potential which determines the masses of the scalar fields is a complicated function determined by the gauging. Here it was not obvious a priori 
that the gauging does not lift some of the flat directions or creates new ones. Thus it is non-trivial that we can model flop and conifold transitions with our quaternionKähler manifolds.

However, it is clear that a LEEA based on $X(1+N)$ can only be a toy model, as the hypermultiplet manifolds which actually occur in string and M-theory compactifications are unlikely to be symmetric spaces. Moreover, it is conceivable that integrating out the charged hypermultiplets modifies the couplings of the neutral hypermultiplets, so that the manifolds of the In-picture and the Out-picture are not related by the simple truncation $X(1+N) \rightarrow X(1)$. Yet, the very fact that we find a consistent description of the topological phase transitions shows that while such threshold corrections might modify the couplings, they cannot play an essential role. This is different in the vector multiplet sector, where these corrections are crucial when determining the In-picture LEEA, because the Out-picture LEEA are discontinuous. ${ }^{37}$

In summary, our models are a reasonable approximation of M-theory physics because they (i) define consistent gauged supergravity actions, (ii) have, for an arbitrary number of transition states, the correct properties to model the transitions, (iii) are unique once the hypermultiplet scalar manifold is fixed and (iv) are simple enough to allow for explicit calculations.

The last point is illustrated by studying Kasner cosmological solutions arising in particular examples for flop and conifold transitions. Thereby we investigate both the Out-picture, where the extra light states arising in the transition are integrated out, and in the In-picture, where they are dynamical. In the Out-picture, cosmologies undergoing a flop transition are regular while conifold transitions cannot be realized dynamically. The moduli encoding the volume and shape of the internal manifold typically show run-away behavior and there is no accelerated expansion in

\footnotetext{
${ }^{37}$ In the related case of $S U(2)$ enhancement it was proven in [23] that the Out-picture LEEA cannot be extended to an $S U(2)$ invariant action without taking into account the threshold corrections.
} 
three-space. This changes drastically once all light modes occurring in the transition region are taken to be dynamical. In this case both flop and conifold transitions lead to a smooth variation of the physical quantities across the transition locus, even though the latter interpolate between internal spaces with different Euler numbers. Further the non-trivial scalar potential has profound consequences for moduli stabilization and inflation.

Concerning the moduli stabilization we have seen that the usual picture of runaway behavior can be highly misleading. As soon as we allow all light states to be excited the moduli are dynamically confined to the transition region. Thus the "almost singular" manifolds close to a topological phase transition are dynamically preferred. ${ }^{38}$ This is somewhat surprising, because the potential has still many unlifted flat directions, so that there is no energy barrier which prevents the system from running away. Therefore this effect cannot be predicted by just analyzing the critical points of the superpotential.

The behavior of our cosmological solutions can be qualitatively understood from a thermodynamic analogy. Around the transition locus additional degrees of freedom can be excited. Generically, the available energy is then distributed equally among all the light modes ("thermalization"). Once this has happened it becomes very unlikely (though it remains possible in principle) that the system "finds" the flat directions and "escapes" from the transition region ("entropy beats energy"). This picture is consistent with all the numerical solutions we have looked at: irrespective of the initial conditions the system finally settles down in a state where all the fields either approach constant finite values or oscillate around the transition region with comparable and small amplitudes. For long simulation time one sees "fluctuations from equilibrium", i.e., some mode picks up a bigger share of the energy for a while, but the system eventually thermalizes again. In the case of flop transitions, this fits nicely with the non-hyperbolic character of the fixed point manifold, which implies

\footnotetext{
${ }^{38}$ For a quantitative analysis of this effect for hyperbolic potentials see [77].
} 
that linearized solutions describe oscillations.

In a realistic scenario of moduli stabilization one would of course prefer that the system is damped, so that the moduli are attracted to fixed point values. This is possible for hyperbolic fixed points, which are the generic case in dynamical systems. Obviously, the non-generic feature of our system is the existence of a degenerate family of supersymmetric vacua. It would be interesting to investigate if the lifting of these flat directions makes the fixed point hyperbolic. This would open the possibility of attractor behavior, but also carries the risk of reintroducing run-away behavior.

Let us now discuss what can be learned about inflation. Our In-picture solutions generically exhibit several periods of transient acceleration. Both the analysis of the slow-roll conditions and the study of numerical solutions shows that the amount of acceleration is much too small to account for an inflationary expansion of the early universe. However, our mechanism might still be relevant for the moderate acceleration suggested by current observations.

Again, the behavior of the solutions can be understood qualitatively in terms of properties of the scalar potential. The point is that the potential is only flat along the unlifted directions parametrized by the moduli. Thus it is either flat, but vanishing, or non-vanishing, but steep. This also explains why hybrid inflation $[56,57]$ is not realized in our models, despite the ingredients are present, namely several scalar fields and both flat and steep directions in the scalar potential. To get a considerable amount of inflation, one needs to lift the flat directions gently without making them to steep.

Our construction also avoids the no-go theorem $[78,79,80]$ which excludes de Sitter vacua in ten- or eleven-dimensional supergravity compactified on timeindependent, smooth and compact internal spaces based on the strong energy condition. In both the Out- and the In-picture we compactified on a time-dependent manifold. Moreover, in the In-picture we have gone beyond eleven-dimensional supergravity by including states of wrapped M2-branes, in order to have a sensible 
theory when the internal space becomes singular. But as we have seen, the mere fact that the no-go theorem is circumvented does not automatically lead to the existence of de Sitter solutions or inflation.

This feature is shared by two other approaches for obtaining positive semi-definite potentials from string or M-theory compactifications, namely the inclusion of fluxes and hyperbolic compactifications. As was pointed out in $[76,81]$, it is a common feature of these potentials that cosmological solutions exhibit epochs of accelerated expansion, which generically are not pronounced enough to describe primordial inflation. The inclusion of transition states in singular Calabi-Yau compactifications provides an interesting alternative to naturally obtain such potentials from string or M-theory.

In summary we see that the dynamics of the transition states is interesting and relevant, but can only be part of the solution of the problems of moduli stabilization and inflation. The first step to extend our work is to consider more general gaugings of our five-dimensional model. Here the detailed study of Kasner solutions performed in this thesis will be helpful, because we know which kinds of contributions to the equations of motion are needed in order to enhance the periods of acceleration and to stabilize the moduli. Once gaugings which lead to interesting cosmological solutions are found, one should clarify whether these can be derived from string or M-theory where they correspond to adding fluxes or branes. This framework also allows to address supersymmetry breaking.

Another direction is to work out to which extent our results can hold independently of the detailed form of the hypermultiplet metric. In particular, one can use the fact that a Calabi-Yau manifold has a flop transition in order to constrain the hypermultiplet metric in the In-picture. Yet another direction is to consider topological transitions other than flop and conifold transitions, where one also gets non-abelian gauge symmetry enhancement. Finally, one should investigate fourdimensional cosmologies, for example in the context of type II compactifications 
on Calabi-Yau threefolds. Here the main new complication is the vector multiplet sector, which no longer is controlled by a simple cubic polynomial, but by a general holomorphic prepotential. Ultimately, all these directions need to be put together to find realistic four-dimensional cosmologies in compactifications which include the effects of flux, branes and transition states. 


\section{References}

[1] M.B. Green, J.H. Schwarz, E. Witten, Superstring theory, two vols., Cambridge University Press (1987).

[2] J. Polchinski, String Theory, two vols., Cambridge University Press (2000).

[3] C.V. Johnson, D-Branes, Cambridge University Press (2003).

[4] A. Strominger, Nucl. Phys. B451 (1995) 96, hep-th/9504090.

[5] E. Witten, Nucl. Phys. B471 (1996) 195, hep-th/9603150.

[6] B.R. Greene, in Fields, strings and duality, Boulder (1996) 543, hepth/9702155.

[7] P.S. Aspinwall, B.R. Greene, D.R. Morrison, Nucl. Phys. B416 (1994) 414, hep-th/9309097.

[8] E. Witten, Nucl. Phys. B403 (1993) 159, hep-th/9301042.

[9] B.R. Greene, D.R. Morrison, A. Strominger, Nucl. Phys. B451 (1995) 109, hep-th/9504145.

[10] I. Gaida, S. Mahapatra, T. Mohaupt, W.A. Sabra, Class. Quant. Grav. 16 (1999) 419, hep-th/9807014.

[11] B.R. Greene, K. Schalm, G. Shiu, J. Math. Phys. 42 (2001) 3171, hepth/0010207.

[12] T. Mohaupt, Fortsch. Phys. 51 (2003) 787, hep-th/0212200.

[13] M. Brändle, A. Lukas, Phys. Rev. D68 (2003) 024030, hep-th/0212263.

[14] M. Bershadsky, V. Sadov, C. Vafa, Nucl. Phys. B463 (1996) 398, hepth/9510225.

[15] A. Klemm, P. Mayr, Nucl. Phys. B469 (1996) 37, hep-th/9601010.

[16] S. Katz, D.R. Morrison, R. Plesser, Nucl. Phys. B477 (1996) 105, hepth/9601108.

[17] J. Polchinski, A. Strominger, Phys. Lett. B388 (1996) 736, hep-th/9510227. T.R. Taylor, C. Vafa, Phys. Lett. B474 (2000) 130, hep-th/9912152. 
[18] G. Curio, A. Klemm, D. Lüst, S. Theisen, Nucl. Phys. B609 (2001) 3, hepth/0012213.

[19] L. Kofman, A. Linde, X. Liu, A. Maloney, L. McAllister, E. Silverstein, Beauty is attractive: moduli trapping at enhanced symmetry points, hep-th/0403001.

[20] P. Mayr, Nucl. Phys. B593 (2001) 99, hep-th/0003198.

[21] S.B. Giddings, S. Kachru, J. Polchinski, Phys. Rev. D66 (2002) 106006, hepth/0105097.

[22] J.L. Feng, J. March-Russel, S. Sethi, F. Wilczek, Nucl. Phys. B602 (2001) 307, hep-th/0005276.

[23] T. Mohaupt, M. Zagermann, JHEP 12 (2001) 026, hep-th/0109055.

[24] J. Louis, T. Mohaupt, M. Zagermann, JHEP 02 (2003) 053, hep-th/0301125.

[25] M. Wijnholt, S. Zhukov, Nucl. Phys. B639 (2002) 343, hep-th/0110109.

[26] J. Bagger, E. Witten, Nucl. Phys. B222 (1983) 1.

[27] M. Günaydin, G. Sierra, P.K. Townsend, Nucl. Phys. B242 (1984) 244; Nucl. Phys. B253 (1985) 573.

[28] M. Günaydin, M. Zagermann, Nucl. Phys. B572 (2000) 131, hep-th/9912027.

[29] A. Ceresole, G. Dall'Agata, Nucl. Phys. B585 (2000) 143, hep-th/0004111.

[30] J.R. Ellis, M. Günaydin, M. Zagermann, JHEP 11 (2001) 024, hepth/0108094.

[31] E. Bergshoeff, S. Cucu, T. de Wit, J. Gheerardyn, S. Vandoren, A. Van Proeyen, $\mathcal{N}=2$ supergravity in five dimensions revisited, hepth/0403045.

[32] A.C. Cadavid, A. Ceresole, R. D'Auria, S. Ferrara, Phys. Lett. B357 (1995) 76, hep-th/9506144. G. Papadopoulos, P.K. Townsend, Phys. Lett. B357 (1995) 300, hep-th/9506150.

[33] I. Antoniadis, R. Minasian, S. Theisen, P. Vanhove, Class. Quant. Grav. 20 (2003) 5079, hep-th/0307268. 
[34] L. Anguelova, M. Roček, S. Vandoren, Quantum corrections to the universal hypermultiplet and superspace, hep-th/0402132.

[35] M. Davidse, U. Theis, S. Vandoren, Five-brane instanton corrections to the universal hypermultiplet, hep-th/0404147.

[36] S. Cecotti, S. Ferrara, L. Girardello, Int. J. Mod. Phys. A4 (1989) 2475.

[37] S. Ferrara, S. Sabharwal, Nucl. Phys. B332 (1990) 317.

[38] P. Aspinwall, in Strings, branes and gravity, Boulder (1999) 723, hepth/0001001.

[39] A. Swann, Math. Ann. 289 (1991) 421.

[40] B. de Wit, M. Roček, S. Vandoren, JHEP 02 (2001) 039, hep-th/0101161.

[41] B. de Wit, M. Roček, S. Vandoren, Phys. Lett. B511 (2001) 302, hepth/0104215.

[42] L. Anguelova, M. Roček, S. Vandoren, JHEP 05 (2002) 064, hep-th/0202149.

[43] J.E. Lidsey, D. Wands, E.J. Copeland, Phys. Rept. 337 (2000) 343, hepth/9909061.

[44] D.N. Spergel et al., Astrophys. J. Suppl. 148 (2003) 175, astro-ph/0302209.

[45] S. Perlmutter et al., New constraints on $\Omega_{M}, \Omega_{\Lambda}$, and $W$ from an independent set of eleven high-redshift supernovae observed with HST, astro-ph/0309368.

[46] A. Riess et al., Astron. J. 116 (1998) 1009, astro-ph/9805201.

[47] M. Trodden, S.M. Carroll, TASI lectures: introduction to cosmology, astro$\mathrm{ph} / 0401547$.

[48] A. Linde, Particle physics and inflationary cosmology, Harwood, Chur, Switzerland (1990).

[49] A.R. Liddle, D.H. Lyth, Cosmological inflation and large-scale structure, Cambridge University Press (2000).

[50] A. Riess et al., Type IA supernova discoveries at $z>1$ from the Hubble Space Telescope: evidence for past deceleration and constraints on dark enery evolution, astro-ph/0402512. 
[51] S. Kachru, R. Kallosh, A. Linde, S.P. Trivedi, Phys. Rev. D68 (2003) 046005, hep-th/0301240.

[52] S. Kachru, R. Kallosh, A. Linde, J. Maldacena, L. McAllister, S.P. Trivedi, JCAP 10 (2003) 013, hep-th/0308055.

[53] C.P. Burgess, R. Kallosh, F. Quevedo, JHEP 10 (2003) 056, hep-th/0309187.

[54] R. Brustein, S.P. de Alwis, E.G. Novak, Phys. Rev. D68 (2003) 043507, hepth/0212344.

[55] R. Brustein, S.P. de Alwis, Moduli potentials in sting compactifications with fluxes: mapping the discretuum, hep-th/0402088.

[56] A. Linde, Phys. Lett. B259 (1991) 38; Phys. Rev. D49 (1994) 748.

[57] A. Linde, A. Riotto, Phys. Rev. D56 (1997) 1841, hep-ph/9703209.

[58] F. Quevedo, Lectures on string/brane cosmology, Class. Quant. Grav. 19 (2002) 5721, hep-th/0210292.

[59] A. Ceresole, G. Dall'Agata, R. Kallosh, A. Van Proeyen, Phys. Rev. D64 (2001) 104006, hep-th/0104056.

[60] B. de Wit, B. Kleijn, S. Vandoren, Nucl. Phys. B568 (2000) 47, hepth/9909228.

[61] K. Galicki, Commun. Math. Phys. 108 (1987) 117. R. D'Auria, S. Ferrara, P. Fré, Nucl. Phys. B359 (1991) 705. L. Andrianopoli, M. Bertolini, A. Ceresole, R. D’Auria, S. Ferrara, P. Fré, T. Magri, J. Geom. Phys. 23 (1997) 111, hep-th/9605032. L. Andrianopoli, M. Bertolini, A. Ceresole, R. D'Auria, S. Ferrara, P. Fré, Nucl. Phys. B476 (1996) 397, hep-th/9603004.

[62] R. D'Auria, S. Ferrara, JHEP 05 (2001) 034, hep-th/0103153.

[63] P.K. Townsend, Phys. Lett. B148 (1984) 55.

[64] D.V. Alekseevsky, V. Cortés, C. Devchand, A. Van Proeyen, Commun. Math. Phys. 238 (2003) 525, hep-th/0109094.

[65] I. Antoniadis, B. Pioline, Int. J. Mod. Phys. A12 (1997) 4907, hep-th/9607058.

[66] T. Hübsch, Calabi-Yau manifolds, World Scientific, 1992. 
[67] P.M.H. Wilson, Invent. Math. 107 (1992) 561.

[68] L. Alvarez-Gaume, S.F. Hassan, Fortsch. Phys. 45 (1997) 159, hepth/9701069.

[69] N. Hitchin, A. Karlhede, U. Lindström, M. Roček, Commun. Math. Phys. 108 (1987) 535 .

[70] J. Marsden, A. Weinstein, Rep. Math. Phys. 5 (1974) 121.

[71] A. Lukas, B. Ovrut, K. Stelle, D. Waldram, Nucl. Phys. B552 (1999) 246, hep-th/9806051.

[72] C. Mayer, T. Mohaupt, Class. Quant. Grav. 21 (2004) 1879, hep-th/0312008.

[73] D.V. Anosov, S.K. Aranson, I.U. Bronshtein, V.Z. Grines, Y.S. Il'yashenko, Ordinary differential equations and smooth dynamical systems, Springer, (1997).

[74] W. Hurewicz, Lectures on ordinary differential equations, Dover, New York, (1990).

[75] M. Sasaki, E.D. Stewart, Prog. Theor. Phys. 95 (1996) 71, astro-ph/9507001.

[76] R. Emparan, J. Garriga, JHEP 05 (2003) 028, hep-th/0304124.

[77] R. Helling, Beyond eikonal scattering in M(atrix)-theory, hep-th/0009134.

[78] B. de Wit, D.J. Smit, N.D. Hari Dass, Nucl. Phys. B283 (1987) 165.

[79] G.W. Gibbons, Aspects of supergravity theories in Supergravity and related topics, eds. F. del Aguila, J.A. de Azcárraga, L.E. Ibanez, World Scientific (1985).

[80] J.M. Maldacena, C. Nuñez, Int. J. Mod. Phys. A16 (2001) 822, hepth/0007018.

[81] P.K. Townsend, Cosmic acceleration and M-theory, hep-th/0308149.

[82] J. Louis, J. Sonnenschein, S. Theisen, S. Yankielowicz, Nucl. Phys. B480 (1996) 185, hep-th/9606049.

[83] A. Chou, R. Kallosh, J. Rahmfeld, S.-J. Rey, M. Shmakova, W.K. Wong, Nucl. Phys. B508 (1997) 147, hep-th/9704142. 


\section{Publications}

[FS10] T. Mohaupt and F. Saueressig, Dynamical conifold transitions in M-theory, in preparation.

[FS9] L. Järv, T. Mohaupt and F. Saueressig, Quintessence cosmologies with a double exponential potential, submitted to JCAP [arXiv:hep-th/0403063].

[FS8] L. Järv, C. Mayer, T. Mohaupt and F. Saueressig, Space-time singularities and the Kähler cone, contributed to the proceedings of the 36th Symposium Ahrenshoop, Berlin, August 26-30, 2003 [arXiv:hep-th/0312195].

[FS7] V. Cortés, C. Mayer, T. Mohaupt and F. Saueressig, Special geometry of Euclidean supersymmetry. I. vector multiplets, JHEP 03 (2004) 028 [arXiv:hepth/0312001].

[FS6] M. Reuter and F. Saueressig, Nonlocal quantum gravity and the size of the universe, contributed to the proceedings of the 36th Symposium Ahrenshoop, Berlin, August 26-30, 2003 [arXiv:hep-th/0311056].

[FS5] L. Järv, T. Mohaupt and F. Saueressig, Singular compactifications and cosmology, in "Symmetries beyond the standard model" , Portoroz (2003) 254 [arXiv:hep-th/0311016].

[FS4] L. Järv, T. Mohaupt and F. Saueressig, M-theory cosmologies from singular Calabi-Yau compactifications, JCAP 02 (2004) 012 [arXiv:hep-th/0310174].

[FS3] L. Järv, T. Mohaupt and F. Saueressig, Effective supergravity actions for flop transitions, JHEP 12 (2003) 047 [arXiv:hep-th/0310173].

[FS2] M. Reuter and F. Saueressig, A class of nonlocal truncations in Quantum Einstein Gravity and its renormalization group behavior, Phys. Rev. D66 (2002) 125001 [arXiv:hep-th/0206145].

[FS1] M. Reuter and F. Saueressig, Renormalization group flow of quantum gravity in the Einstein-Hilbert truncation, Phys. Rev. D65 (2002) 065016 [arXiv:hepth/0110054]. 


\section{A Vector multiplet sector I: one multiplet}

The simplest In-picture LEEA describing a conifold transition contains two charged hypermultiplets and was constructed in subsection 5.1. The vector multiplet sector of this action consists of a single vector multiplet. For convenience we collect all the relevant properties of this sector in this appendix.

Following [30], the prepotential (2.3) of the most general one vector multiplet scalar manifold can be brought to the standard form

$$
\mathcal{V}=\left(h^{0}\right)^{3}-\frac{3}{2} h^{0}\left(h^{1}\right)^{2}+c\left(h^{1}\right)^{3}=1
$$

where $c$ denotes an arbitrary real constant. The value $c=-\frac{1}{\sqrt{2}}$ corresponds to the manifold $O(1,1)$ considered in [59] while the (Out-picture) prepotential

$$
\mathcal{V}=\frac{3}{8} U^{3}+\frac{1}{2} U T^{2}=1
$$

which arises from Calabi-Yau compactifications on $T^{2} \times K 3$ [82] can be mapped to the case $|c|>\frac{1}{\sqrt{2}}$.

The metric coupling the vector field kinetic terms is obtained from eq. (2.4) and reads

$$
a_{I J}=\left[\begin{array}{ll}
a_{00} & a_{01} \\
a_{10} & a_{11}
\end{array}\right]
$$

with entries

$$
\begin{aligned}
& a_{00}=-2 h^{0}+3\left(\left(h^{0}\right)^{2}-\frac{1}{2}\left(h^{1}\right)^{2}\right)^{2}, \\
& a_{01}=h^{1}-\frac{3 h^{1}}{2}\left(2\left(h^{0}\right)^{2}-\left(h^{1}\right)^{2}\right)\left(h^{0}-c h^{1}\right), \\
& a_{11}=h^{0}-2 c h^{1}+3\left(h^{0} h^{1}-c\left(h^{1}\right)^{2}\right)^{2} .
\end{aligned}
$$

Observe that at the conifold point, $h^{0}=1, h^{1}=0$, this becomes the two-by-two 
unit matrix. Hence the two vector field kinetic terms decouple and have standard normalization at this point.

To fully specify the action, we also need a parametrization of the vector multiplet scalar manifold. Here it is convenient to introduce the vector multiplet scalar field $\phi$ as the homogeneous coordinate

$$
\phi=\frac{h^{1}}{h^{0}}
$$

Solving this relation for $h^{1}$ and substituting the resulting expression into the prepotential (A.1), it is straightforward to calculate $h^{I}(\phi)$ introduced in eq. (2.3):

$$
h^{0}(\phi)=\left(1-\frac{3}{2} \phi^{2}+c \phi^{3}\right)^{-1 / 3}, \quad h^{1}(\phi)=\phi\left(1-\frac{3}{2} \phi^{2}+c \phi^{3}\right)^{-1 / 3}
$$

The vector multiplet scalar manifold (2.6) is then given by

$$
g_{x y}=\frac{3\left(2-4 c \phi+\phi^{2}\right)}{\left(2-3 \phi^{2}+2 c \phi^{3}\right)^{2}}
$$

At the conifold point $h^{1}(\phi)=0 \leftrightarrow \phi=0$ this metric is regular $\left.g_{x y}\right|_{\phi=0}=\frac{3}{2}$. The coordinate range of $\phi$ is bounded by the values $\phi_{-}^{\text {crit }}$ and $\phi_{+}^{\text {crit }}$, at which the metric becomes zero or infinite. For $|c|<\frac{1}{\sqrt{2}}$ the metric diverges at both $\phi_{-}^{\text {crit }}$ and $\phi_{+}^{\text {crit }}$ while for $|c| \geq \frac{1}{\sqrt{2}}$ we encounter one zero and one infinity bounding the coordinate range of $\phi$. For the special case $c=0$ considered in section 8 we have $\phi_{ \pm}^{\text {crit }}= \pm \sqrt{\frac{2}{3}}$ implying the corresponding vector multiplet scalar manifold is given by the interval ]$-\sqrt{\frac{2}{3}}, \sqrt{\frac{2}{3}}[$. Note that even though the coordinate length of this interval is finite, the boundaries $\phi_{ \pm}^{\text {crit }}$ are at infinite geodesic distance from the origin. 


\section{B Vector multiplet sector II: $n_{V}$ multiplets}

The construction of the In-picture LEEA describing a general conifold transition in subsection 5.2 required specifying an explicit vector multiplet sector containing $n_{V}$ vector multiplets. In this appendix we derive the properties of the vector field and vector multiplet scalar metrics needed in this construction. The calculation is analogous to the one vector multiplet case considered in appendix A, but due to the increased number of vector multiplets somewhat more involved.

We start by specifying the prepotential (2.3) to be

$$
\mathcal{V}=\left(h^{0}\right)^{3}-\frac{3}{2} h^{0} \sum_{x=1}^{n_{V}}\left(h^{x}\right)^{2}=1
$$

where $x, y, z=1, \ldots, n_{V}$ counts the vector multiplets. The prepotential is again given in the standard form [30], but is not the most general one, as we have chosen $C_{x y z}=0$ in order to keep the calculation manageable.

The metric coupling the kinetic terms of the vector fields is obtained from eq. $(2.4)$

$$
a_{I J}=\left[\begin{array}{ll}
a_{00} & a_{0 x} \\
a_{x 0} & a_{x y}
\end{array}\right]
$$

with the entries being

$$
\begin{aligned}
& a_{00}=-2 h^{0}+3\left(\left(h^{0}\right)^{2}-\frac{1}{2} \sum_{z=1}^{n_{V}}\left(h^{z}\right)^{2}\right)^{2}, \\
& a_{0 x}=h^{x}-3 h^{0} h^{x}\left(\left(h^{0}\right)^{2}-\frac{1}{2} \sum_{z=1}^{n_{V}}\left(h^{z}\right)^{2}\right) \\
& a_{x y}=h^{0} \delta_{x y}+3\left(h^{0}\right)^{2} h^{x} h^{y} .
\end{aligned}
$$

In order to have an explicit parametrization of the vector multiplet scalar manifold 
we introduce homogeneous coordinates

$$
\phi^{x}=\frac{h^{x}}{h^{0}}, \quad x=1, \ldots, n_{V}
$$

Solving these relations for $h^{x}$ and substituting the resulting expressions into the prepotential (B.1) the $h^{I}$ become functions of the vector multiplet scalars $\phi^{x}$ :

$$
h^{0}(\phi)=\left(1-\frac{3}{2} \sum_{z=1}^{n_{V}}\left(\phi^{z}\right)^{2}\right)^{-1 / 3}, h^{x}(\phi)=\phi^{x}\left(1-\frac{3}{2} \sum_{z=1}^{n_{V}}\left(\phi^{z}\right)^{2}\right)^{-1 / 3} .
$$

These relations are used to calculate the components of the vector multiplet scalar metric $(2.6)$

$$
g_{x y}= \begin{cases}\frac{3\left(1+\frac{1}{2}\left(\phi^{x}\right)^{2}-\frac{3}{2} \sum_{z \neq x}^{n} V\left(\phi^{z}\right)^{2}\right)}{2\left(1-\frac{3}{2} \sum_{z=1}^{n} V\left(\phi^{z}\right)^{2}\right)^{2}}, & \text { for } x=y, \\ \frac{6 \phi^{x} \phi^{y}}{\left(1-\frac{3}{2} \sum_{z=1}^{n} V\left(\phi^{z}\right)^{2}\right)^{2}}, & \text { for } x \neq y .\end{cases}
$$

In order to find the mass matrix on the Higgs branch we further need the inverse vector multiplet scalar metric $g^{x y}$ whose components are given by

$$
g^{x y}= \begin{cases}\frac{2\left(1-\frac{3}{2}\left(\phi^{x}\right)^{2}+\frac{1}{2} \sum_{z \neq x}^{n}{ }^{n}\left(\phi^{z}\right)^{2}\right)\left(1-\frac{3}{2} \sum_{z=1}^{n}{ }^{n}\left(\phi^{z}\right)^{2}\right)}{3\left(1+\frac{1}{2} \sum_{z=1}^{n} V\left(\phi^{z}\right)^{2}\right)}, & \text { for } x=y, \\ -\frac{4 \phi^{x} \phi^{y}\left(1-\frac{3}{2} \sum_{z=1}^{n} V\left(\phi^{z}\right)^{2}\right)}{\left.3\left(1+\frac{1}{2} \sum_{z=1}^{n} \phi^{z}\right)^{2}\right)}, & \text { for } x \neq y .\end{cases}
$$

The vacuum conditions on the Higgs branch generically fix a subset $\phi^{\alpha}, \alpha=$ $1, \ldots, N-r \leq n_{V}$, to be zero (cf. eq. (5.15) for the case $r=1$ ). By virtue of the relation (B.5) this corresponds to the $h^{\alpha}$ parametrizing the volume of the contracting cycles being zero. Restricting $g^{x y}$ given above to $\phi^{\alpha}=0$, the resulting expression becomes block diagonal

$$
\left.g^{x y}\right|_{\phi^{\alpha}=0}=\left[\begin{array}{cc}
a \mathbb{1}_{(N-r) \times(N-r)} & 0 \\
0 & g
\end{array}\right]
$$


where

$$
a:=\frac{2}{3}\left(1-\frac{3}{2} \sum_{z=m+1}^{n_{V}}\left(\phi^{z}\right)^{2}\right),
$$

and $g$ is to be understood as $\left.g^{x y}\right|_{\phi^{\alpha}=0}$ with $x, y=N-r+1, \ldots, n_{V}$. This data completely determines the vector multiplet sector of the conifold model discussed in subsection 5.2 and enables us to explicitly calculate the vector multiplet scalar masses on the Higgs branch. 


\section{Vector multiplet sector III: the $\mathbb{F}_{1}$-model}

In subsection 4.1 as well as in the sections 6 and 7 we used an explicit example of a CY threefold $X$ with a flop transition involving a single isolated holomorphic curve. In this case one charged hypermultiplet becomes massless at the transition locus. The corresponding CY space is known as the 'elliptic fibration over the first Hirzebruch surface', or $\mathbb{F}_{1}$-model for short, and all its relevant properties can be found in $[82,83]$. In order to make this work self-contained, we summarize these properties in this appendix.

The extended Kähler cone of the $\mathbb{F}_{1}$-model consists of two Kähler cones which were called regions II and III in [83]. In terms of adapted parametrizations, $h^{I}>0$ and $\tilde{h}^{I}>0$, the prepotentials $(2.3)$ of these regions are given by $[82]^{39}$

$$
\begin{aligned}
\mathcal{V}_{(\mathrm{II})}= & 6\left(h^{0}\right)^{3}+9\left(h^{1}\right)^{3}+27\left(h^{0}\right)^{2} h^{1}+27 h^{0}\left(h^{1}\right)^{2}+9\left(h^{0}\right)^{2} h^{2}+9\left(h^{1}\right)^{2} h^{2} \\
& +3 h^{0}\left(h^{2}\right)^{2}+3 h^{1}\left(h^{2}\right)^{2}+18 h^{0} h^{1} h^{2}=1,
\end{aligned}
$$

and

$$
\mathcal{V}_{(\mathrm{III})}=8\left(\tilde{h}^{0}\right)^{3}+9\left(\tilde{h}^{0}\right)^{2} \tilde{h}^{1}+3 \tilde{h}^{0}\left(\tilde{h}^{1}\right)^{2}+6\left(\tilde{h}^{0}\right)^{2} \tilde{h}^{2}+6 \tilde{h}^{0} \tilde{h}^{1} \tilde{h}^{2}=1
$$

The flop transition occurs at $h^{1} \rightarrow 0$ and $\tilde{h}^{2} \rightarrow 0$, respectively.

To analyze the transition it is convenient to introduce variables $T, U, W$, which can be used in both regions. They are given by

$$
\begin{array}{lll}
6^{1 / 3} h^{0}=W, & 6^{1 / 3} h^{1}=U-W, & 6^{1 / 3} h^{2}=T-\frac{3}{2} U, \\
6^{1 / 3} \tilde{h}^{0}=U, & 6^{1 / 3} \tilde{h}^{1}=T-\frac{1}{2} U-W, & 6^{1 / 3} \tilde{h}^{2}=W-U .
\end{array}
$$

These formulae also encode the mutual relation between the adapted variables $h^{I}$

\footnotetext{
${ }^{39}$ Note that the $t^{I}$ appearing in $[82,83]$ are related to the $h^{I}$ by $h^{I}=6^{-1 / 3} t^{I+1}$.
} 
and $\tilde{h}^{I}$. In terms of $T, U, W$, the prepotentials (C.1) and (C.2) become

$$
\begin{aligned}
& \mathcal{V}_{(\mathrm{II})}=\frac{3}{8} U^{3}+\frac{1}{2} U T^{2}-\frac{1}{6} W^{3}=1 \\
& \mathcal{V}_{(\mathrm{III})}=\frac{5}{24} U^{3}+\frac{1}{2} U^{2} W-\frac{1}{2} U W^{2}+\frac{1}{2} T^{2} U=1 .
\end{aligned}
$$

The flop line is located at $U=W$. Comparing these prepotentials, we find that they differ by

$$
\mathcal{V}_{(\mathrm{II})}-\mathcal{V}_{(\mathrm{III})}=\frac{1}{6}(U-W)^{3}
$$

This discontinuity in the triple intersection numbers $C_{I J K}$ exactly matches the contribution arising from integrating out one charged hypermultiplet [5].

To describe the vector multiplet moduli space corresponding to these regions, we solve the constraints (C.4) for $T$

$$
\begin{aligned}
T_{\text {(II) }}(U, W) & =\left(\frac{24+4 W^{3}-9 U^{3}}{12 U}\right)^{1 / 2}, \\
T_{(\mathrm{III})}(U, W) & =\left(\frac{24+12 U W^{2}-12 U^{2} W-5 U^{3}}{12 U}\right)^{1 / 2},
\end{aligned}
$$

and take $U$ and $W$ as independent scalar fields which parametrize the vector multiplet scalar manifolds. The metrics $g_{x y}^{(\mathrm{II})}$ and $g_{x y}^{(\mathrm{III})}$ of these manifolds are obtained from the relations $(2.4),(2.5)$ and (2.6). Substituting $h^{I}(\phi)=T(U, W), U, W$ and $\phi^{x}=U, W$ into these formulae, we find

$$
\begin{aligned}
g_{x y}^{(\mathrm{II})}= & {\left[\begin{array}{cc}
\frac{9 U^{3} W^{3}+54 U^{3}-12 W^{3}-36-W^{6}}{2 U^{2}\left(9 U^{3}-4 W^{3}-24\right)} & -\frac{W^{2}\left(9 U^{3}-W^{3}-6\right)}{2 U\left(9 U^{3}-4 W^{3}-24\right)} \\
-\frac{W^{2}\left(9 U^{3}-W^{3}-6\right)}{2 U\left(9 U^{3}-4 W^{3}-24\right)} & \frac{W\left(9 U^{3}-W^{3}-24\right)}{2\left(9 U^{3}-4 W^{3}-24\right)}
\end{array}\right], } \\
g_{x y}^{(\mathrm{III})}= & {\left[\begin{array}{cc}
\frac{18-15 U^{3}-18 U^{2} W+12 U W^{2}-4 U^{4} W^{2}}{U^{2}\left(24+12 U W^{2}-12 U^{2} W-5 U^{3}\right)} & \frac{6 W-9 U+4 U^{3} W}{24+12 U W^{2}-12 U^{2} W-5 U^{3}} \\
\frac{6 W-9 U+4 U^{3} W}{24+12 U W^{2}-12 U^{2} W-5 U^{3}} & \frac{4 U\left(3-U^{3}\right)}{24+12 U W^{2}-12 U^{2} W-5 U^{3}}
\end{array}\right] . }
\end{aligned}
$$

These metrics are non-degenerate at the flop line $U=W$, and can be connected continuously. 

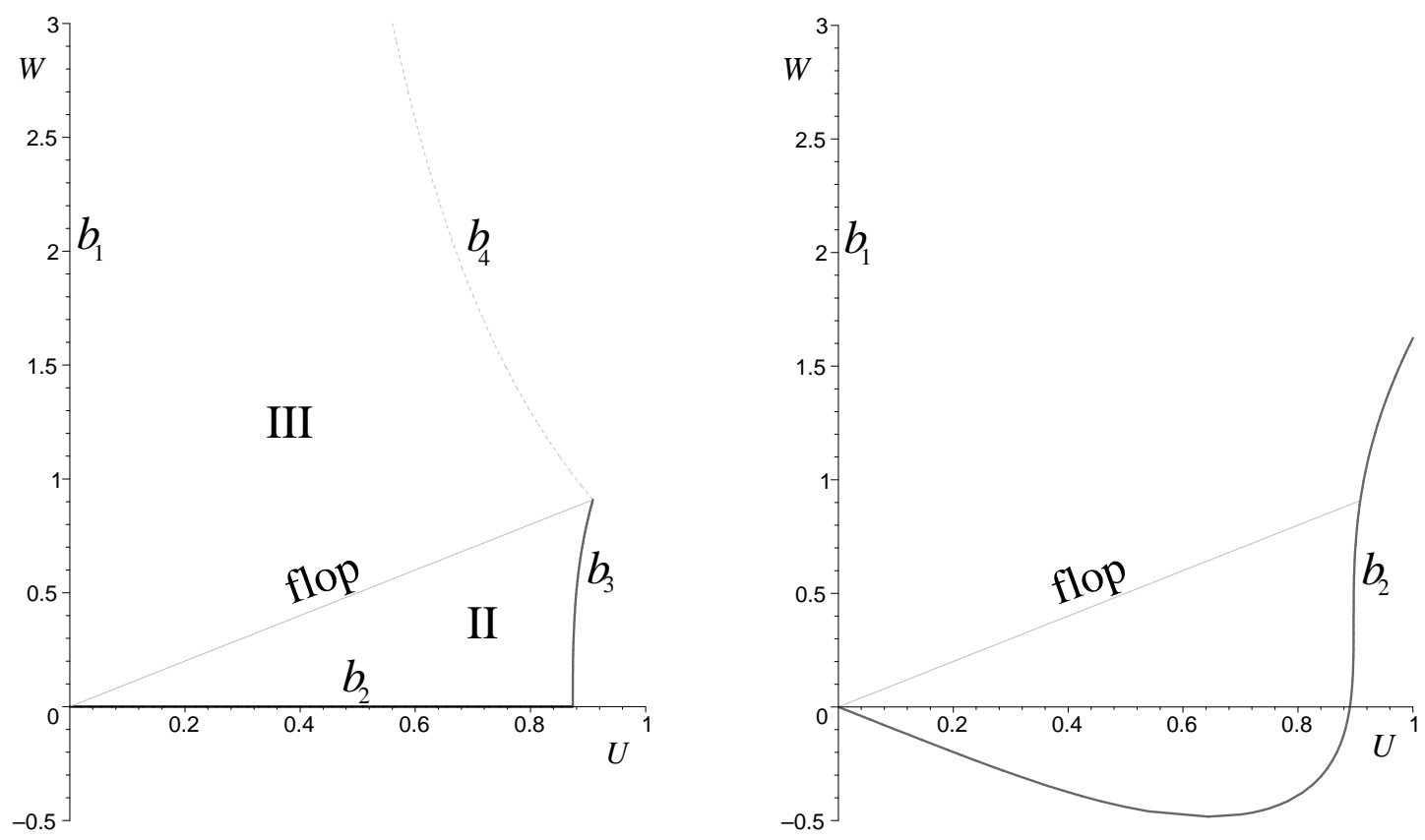

Figure 13: Comparison between the vector multiplet scalar manifolds in the Out- (left) and In-picture (right). The gray line labeled "flop" indicates the locus of the flop transition, $U=W$. The ' $b_{1}$ ', ' $b_{2}$ ', ' $b_{3}$ ' and ' $b_{4}$ ' denote the other boundaries of the scalar manifolds. The location of these boundaries is different in the Out- and the In-picture.

The extended Kähler cone of the $\mathbb{F}_{1}$-model is shown in the left diagram of figure 13. Besides the flop line, this diagram displays additional boundaries labeled ' $b_{1}$ ', ' $b_{2}$ ', ' $b_{3}$ ' and ' $b_{4}$ ', which have the following meaning:

- The boundary $b_{1}$ corresponds to $\tilde{h}^{0} \rightarrow 0$. The metric on the Kähler cone has an infinite eigenvalue. In the full Kähler cone this limit corresponds to the CY volume becoming zero. However, the vector multiplet manifold of the five-dimensional supergravity theory corresponds to a hypersurface of the Kähler cone, obtained by keeping the total volume constant. In this subspace the singularity takes a different form: while some two-cycles collapse, others diverge, such that the total volume remains at a fixed finite value [12].

- The boundaries $b_{2}$ and $b_{3}$ correspond to $h^{0} \rightarrow 0$ and $h^{2} \rightarrow 0$, respectively. Here the metric on the Kähler cone degenerates and has a zero eigenvalue. 
In the microscopic picture a surface is contracted to a point and one obtains tensionless strings. Furthermore, the line $h^{0} \rightarrow 0$ is the fixed volume section of the Kähler cone arising from the elliptic fibration over $\mathbb{P}^{2}$. Since one divisor has been blown down, this space has one Kähler modulus less. This boundary component has been called region I in [83]. Its prepotential is given in eq. (A.2).

- The $b_{4}$ boundary corresponds to $\tilde{h}^{1} \rightarrow 0$. The metric on the Kähler cone is regular. At this boundary one obtains $S U(2)$-enhancement. ${ }^{40}$

We now construct the vector multiplet scalar manifold for the In-picture Lagrangian. The corresponding prepotential is determined by the orbit sum rule (2.38). Taking the average of the prepotentials $\mathcal{V}_{(\mathrm{II})}$ and $\mathcal{V}_{(\mathrm{III})}$, one finds

$$
\begin{aligned}
\mathcal{V}_{(\text {In })} & =\frac{1}{2}\left(\mathcal{V}_{(\mathrm{II})}+\mathcal{V}_{(\mathrm{III})}\right) \\
& =\frac{7}{24} U^{3}+\frac{1}{2} U T^{2}-\frac{1}{12} W^{3}+\frac{1}{4} U^{2} W-\frac{1}{4} U W^{2}=1 .
\end{aligned}
$$

The metric $g_{x y}^{(\mathrm{In})}$ is obtained analogously to the Out-picture metrics. Solving the constraint (C.8) for $T$, we acquire

$$
T_{(\text {In) }}(U, W)=\frac{1}{2}\left(\frac{24-7 U^{3}+2 W^{3}-6 U^{2} W+6 U W^{2}}{3 U}\right)^{1 / 2} .
$$

Using the definitions (2.4) and (2.6), the components of the In-picture vector multiplet scalar metric

$$
g_{x y}^{(\mathrm{In})}=\left[\begin{array}{ll}
g_{U U} & g_{W U} \\
g_{W U} & g_{W W}
\end{array}\right]
$$

\footnotetext{
${ }^{40}$ This boundary is one of the models where the corresponding In-picture Lagrangian has been worked out in [23].
} 
are

$$
\begin{gathered}
g_{U U}=\frac{1}{8 U^{2} K}\left(144-6 W^{4} U^{2}+48 U W^{2}+4 W^{5} U-72 U^{2} W\right. \\
\left.-14 U^{3} W^{3}-17 U^{4} W^{2}-168 U^{3}+24 W^{3}+W^{6}\right) \\
g_{W U}=\frac{1}{8 U K}\left(\begin{array}{c}
24 U W-36 U^{2}-12 W^{2}-W^{5}-4 W^{4} U \\
\left.\quad+6 U^{2} W^{3}+14 U^{3} W^{2}+17 U^{4} W\right)
\end{array}\right. \\
g_{W W}=\frac{1}{8 K}\left(48(U+W)+4 U W^{3}-6 U^{2} W^{2}-14 U^{3} W+W^{4}-17 U^{4}\right),
\end{gathered}
$$

where

$$
K:=24-7 U^{3}+2 W^{3}-6 U^{2} W+6 U W^{2} .
$$

The corresponding vector multiplet scalar manifold is shown in the second diagram of figure 13. Besides the flop line at $U=W$ where the metric is regular, this diagram shows two additional boundaries, labeled ' $b_{1}$ ' and ' $b_{2}$ '. These have the following meaning:

- The boundary $b_{1}$ corresponds to $U \rightarrow 0$. Here the metric (C.10) has an infinite eigenvalue.

- At the boundary $b_{2}$ the metric $g_{x y}^{(\mathrm{In})}$ degenerates and has a zero eigenvalue. 


\section{The hypermultiplet sector: $X(n-1)$}

In this appendix we carry out the superconformal quotient construction $[40,41,42]$ outlined in section 3 to derive the explicit formulae for the metrics, Killing vectors and moment maps of $X(n-1)$ given in subsection 3.1. We start by constructing the family of metrics (3.2) in subsection D.1 while the results on the Killing vectors (3.6) and moment maps (3.8) are obtained in subsection D.2. An overview of the spaces and the respective coordinates appearing in the construction is given in table 1.

\section{D.1 The metrics of the Wolf spaces $X(n-1)$}

The starting point: flat complex space $\mathbb{C}^{(2 n+2)}$

We start by considering $\mathbb{C}^{(2 n+2)}$ with complex coordinates $z_{+}^{I}, z_{-I}, I=1, \ldots, n+1$. The metric is taken to be Kähler with the Kähler potential being

$$
\chi_{(2 n+2)}:=\eta_{I \bar{J}} z_{+}^{I} \bar{z}_{+}^{\bar{J}}+\eta^{I \bar{J}} z_{-I} \bar{z}_{-\bar{J}}
$$

Here $\eta_{I \bar{J}}=\operatorname{diag}[-, \ldots,-,+,+]$ has indefinite signature $(p, q)$ with $p=n-1$ negative and $q=2$ positive eigenvalues, and $\eta^{I \bar{J}}$ is its inverse. This signature of $\eta$ ensures that the space obtained from the quotient construction is of non-compact type, as required by supergravity. Later on the coordinates associated with the negative eigenvalues of $\eta$ will play the role of the hypermultiplet scalars, while the coordinates with the positive eigenvalues act as gauge compensators.

We now promote $\mathbb{C}^{(2 n+2)}$ to a hyper-Kähler manifold. For this purpose we introduce the coordinates $x^{\tilde{I}}:=\left\{z_{+}^{I}, \bar{z}_{+}^{\bar{I}}, z_{-I}, \bar{z}_{-\bar{I}}\right\}$, with $\tilde{I}=1, \ldots, 4 n+4$. With respect 


\begin{tabular}{cccc}
\hline \hline symbol & space & $\operatorname{dim}_{\mathbb{R}}$ & coordinates \\
\hline $\mathbb{C}^{(2 n+2)}$ & flat complex space & $4 n+4$ & $\left\{z_{+}^{I}, \bar{z}_{+}^{I}, z_{-I}, \bar{z}_{-I}\right\}$ \\
$\mathcal{H}^{(2 n)}$ & HKC over $X(n-1)$ & $4 n$ & $\left\{z_{+}^{\prime a}, \bar{z}_{+}^{\prime a}, z_{-a}^{\prime}, \bar{z}_{-a}^{\prime}\right\}$ \\
$\mathcal{Z}^{(2 n-1)}$ & twistor space & $4 n-2$ & $\left\{v^{i}, \bar{v}^{i}, u_{i}, \bar{u}_{i}, \zeta, \bar{\zeta}\right\}$ \\
$X(n-1)$ & Wolf space & $4 n-4$ & $\left\{v^{i}, \bar{v}^{i}, u_{i}, \bar{u}_{i}\right\}$ \\
\hline \hline
\end{tabular}

Table 1: Summary of the spaces appearing in the superconformal quotient construction of the unitary Wolf spaces $X(n-1)$. The index ranges are given by $I=1, \ldots, n+1, a=1, \ldots, n$ and $i=1, \ldots, n-1$.

to these coordinates, the $S U(2)$ triplet of complex structures is taken to be:

$$
\begin{aligned}
J_{\tilde{J}}^{1 \tilde{I}}= & {\left[\begin{array}{rrrr}
0 & 0 & 0 & \mathbb{1} \\
0 & 0 & \mathbb{1} & 0 \\
0 & -\mathbb{1} & 0 & 0 \\
-\mathbb{1} & 0 & 0 & 0
\end{array}\right], J_{\tilde{J}}^{2 \tilde{I}}=\left[\begin{array}{cccc}
0 & 0 & 0 & i \mathbb{1} \\
0 & 0 & -i \mathbb{1} & 0 \\
0 & -i \mathbb{1} & 0 & 0 \\
i \mathbb{1} & 0 & 0 & 0
\end{array}\right], } \\
J_{\tilde{J}}^{3 \tilde{I}}= & {\left[\begin{array}{cccc}
i \mathbb{1} & 0 & 0 & 0 \\
0 & -i \mathbb{1} & 0 & 0 \\
0 & 0 & i \mathbb{1} & 0 \\
0 & 0 & 0 & -i \mathbb{1}
\end{array}\right] . }
\end{aligned}
$$

Here the entries are $(n+1) \times(n+1)$ dimensional block matrices and $\mathbb{1}$ denotes the corresponding unit matrix. These complex structures satisfy the quaternionic algebra $J^{r} J^{s}=-\mathbb{1} \delta^{r s}+\epsilon^{r s t} J^{t}$, with $r, s, t=1,2,3$ being the $S U(2)$ index. The complex coordinates $z_{+}^{I}, \bar{z}_{+}^{\bar{I}}, z_{-I}$ and $\bar{z}_{-\bar{I}}$ are defined with respect to the canonical complex structure $J^{3}$. The Kähler metric $g_{\tilde{I} \tilde{J}}$ derived from (D.1) is hermitian with respect to all three complex structures, $g\left(J^{r} \cdot, J^{r} \cdot\right)=g(\cdot, \cdot)$.

Instead of working with the basis (D.2), it is more convenient to use $J^{ \pm}:=$ $\frac{1}{2}\left(J^{1} \pm i J^{2}\right)$, since quantities defined with respect to this basis will turn out to be (anti-)holomorphic with respect to $J^{3}$. From these complex structures we obtain 
the following $S U(2)$ triplet of Kähler forms:

$$
\begin{aligned}
& \omega^{3}=-i \eta_{I \bar{J}} \mathrm{~d} z_{+}^{I} \wedge \mathrm{d} \bar{z}_{+}^{\bar{J}}-i \eta^{I \bar{J}} \mathrm{~d} z_{-I} \wedge \mathrm{d} \bar{z}_{-\bar{J}} \\
& \omega^{+}=\mathrm{d} z_{+}^{I} \wedge \mathrm{d} z_{-I}, \quad \omega^{-}=\bar{\omega}^{+}
\end{aligned}
$$

Their components are given by

$$
\Omega_{\tilde{I} \tilde{J}}^{3}=g_{\tilde{I} \tilde{K}} J_{\tilde{J}}^{3 \tilde{K}}, \quad \Omega_{\tilde{I} \tilde{J}}^{+}=g_{\tilde{I} \tilde{K}} J_{\tilde{J}}^{+\tilde{K}} \quad \text { and } \quad \Omega_{\tilde{I} \tilde{K}}^{-}=\bar{\Omega}_{\tilde{I} \tilde{K}}^{+}
$$

respectively. Here the "bar" denotes complex conjugation with respect to $J^{3}$.

Let us now consider the linear action of the $U(n-1,2)$ isometry subgroup:

$$
z_{+}^{I} \rightarrow U_{J}^{I} z_{+}^{J}, \quad z_{-I} \rightarrow\left(U^{-1}\right)_{I}^{J} z_{-J}
$$

With respect to this isometry, the $z_{+}^{I}$ coordinates transform in the fundamental representation of $U(n-1,2)$, while the $z_{-I}$ transform in the complex conjugate representation. Using $\bar{U}_{\bar{J}}^{\bar{I}}=\eta^{\bar{I} K}\left(U^{-1}\right)_{K}^{N} \eta_{N \bar{J}}$ one finds that the Kähler potential (D.1) is invariant under this transformation. In principle the isometry group of (D.1) contains additional generators. But since these do not descend to tri-holomorphic isometries of the hyper-Kähler cone $\mathcal{H}^{(2 n)}$ they do not give rise to isometries of $X(n-1),{ }^{41}$ and will not be considered here. ${ }^{42}$

The Killing vectors of the linearized isometries are given by

$$
k_{+\alpha}^{I}=i t_{\alpha J}^{I} z_{+}^{J}, \quad k_{-\alpha I}=-i t_{\alpha I}^{J} z_{-J} .
$$

Here $\alpha$ numerates the $(n+1)^{2}$ generators of $U(n-1,2), t_{\alpha}{ }_{J}$. To simplify our notation

\footnotetext{
${ }^{41}$ The coset formulation of $X(n-1)$ indicates that the full isometry group of $X(n-1)$ is given by $S U(n-1,2)$. In our approach this $S U(n-1,2)$ arises from the $U(n-1,2)$ above modulo the $U(1)$ gauged in the hyper-Kähler quotient.

${ }^{42}$ The fact that only tri-holomorphic isometries give rise to isometries of the quaternion-Kähler space has been observed in [40].
} 
we will drop the index $\alpha$ in the following. The action of these Killing vectors is triholomorphic, i.e., the Lie derivative with respect to $k$ satisfies $\mathcal{L}_{k} J^{r}=0$ for all three complex structures (D.2). This implies in particular that the Killing vectors are holomorphic with respect to $J^{3}$. Hence we can obtain their components with respect to $\bar{z}_{+}^{\bar{I}}$ and $\bar{z}_{-\bar{I}}$ by complex conjugation of $k_{+}^{I}$ and $k_{-I}$, respectively.

The condition that the vectors $k$ are Killing, $\mathcal{L}_{k} g=0$, as well as tri-holomorphic implies that they are hamiltonian, $\mathcal{L}_{k} \omega^{r}=0$. The last statement provides the integrability condition for the moment maps associated with these isometries. They are obtained as the solution of the equation

$$
\frac{\partial \mu^{r}}{\partial x^{\tilde{I}}}:=\Omega_{\tilde{I} \tilde{J}}^{r} k^{\tilde{J}}
$$

where $r=+,-, 3$. Substituting the Killing vectors (D.6) and the Kähler forms (D.4), these equations are easily integrated and yield:

$$
\begin{aligned}
& \mu^{3}=-\bar{z}_{+}^{\bar{J}} \eta_{\bar{J} I} t^{I}{ }_{K} z_{+}^{K}+z_{-I} t^{I}{ }_{J} \eta^{J \bar{K}} \bar{z}_{-\bar{K}}, \\
& \mu^{+}=-i z_{-I} t^{I}{ }_{J} z_{+}^{J}, \quad \mu^{-}=\bar{\mu}^{+} .
\end{aligned}
$$

Here we omitted the constants of integration, which, in principle, could give rise to Fayet-Iliopoulous terms. Since these terms appear in neither the superconformal theory defined on the level of the hyper-Kähler cone nor in the $D=5$ supergravity action reviewed in subsection 2.1 , the moment maps (D.8) will give rise to the most general moment maps compatible with the action (2.1).

\section{The hyper-Kähler quotient construction of $\mathcal{H}^{(2 n)}$}

We now perform the hyper-Kähler quotient construction of $\mathcal{H}^{(2 n)}$ by taking the quotient of $\mathbb{C}^{(2 n+2)}$ with respect to the $U(1)$ isometry which acts on $z_{+}^{I}$ and $z_{-I}$ by opposite phase transformations. The infinitesimal generator of this isometry is given 
by $t^{I}{ }_{J}=\delta^{I}$. Substituting this generator into (D.8) we find:

$$
\mu^{3}=-\eta_{I \bar{J}} z_{+}^{I} \bar{z}_{+}^{\bar{J}}+\eta^{I \bar{J}} z_{-I} \bar{z}_{-\bar{J}}, \quad \mu^{+}=-i z_{+}^{I} z_{-I}, \quad \mu^{-}=\bar{\mu}^{+}
$$

The quotient is performed by first introducing $U(1)$ invariant coordinates $z_{+}^{\prime I}, z_{-I}^{\prime}$ on $\mathbb{C}^{(2 n+2)}$ and substituting these into the moment maps (D.9). We then set the resulting moment maps to zero and solve these constraints in terms of $z_{+}^{\prime}{ }^{n+1}, z_{-n+1}^{\prime}$ and their complex conjugates. The remaining unconstrained coordinates $z_{+}^{\prime a}, z_{-a}^{\prime}$ with $a=1, \ldots, n$ provide coordinates on $\mathcal{H}^{(2 n)}$. In practice, we choose the primed coordinates as

$$
z_{+}^{\prime I}:=\frac{z_{+}^{I}}{z_{+}^{n+1}}, \quad z_{-I}^{\prime}:=z_{-I} z_{+}^{n+1}
$$

In terms of these coordinates the moment maps (D.9) become

$$
\begin{aligned}
& \mu^{3}=-\eta_{I \bar{J}} z_{+}^{I I} \bar{z}_{+}^{\prime \bar{J}}\left(\bar{z}_{+}^{n+1} z_{+}^{n+1}\right)+\eta^{I \bar{J}} z_{-I}^{\prime} \bar{z}_{-\bar{J}}^{\prime}\left(\bar{z}_{+}^{n+1} z_{+}^{n+1}\right)^{-1}, \\
& \mu^{+}=-i z_{+}^{\prime I} z_{-I}^{\prime}, \quad \mu^{-}=\bar{\mu}^{+} .
\end{aligned}
$$

Setting the moment maps to zero and solving the resulting constraints in terms of $z_{+}^{\prime n+1}, z_{-n+1}^{\prime}$ and their complex conjugates yields

$$
\left(\bar{z}_{+}^{n+1} z_{+}^{n+1}\right)=\left(\frac{\eta^{I \bar{J}} z_{-I}^{\prime} \bar{z}_{-\bar{J}}^{\prime}}{\eta_{I \bar{J}} z_{+}^{\prime I} \bar{z}_{+}^{\prime \bar{J}}}\right)^{1 / 2}, \quad z_{+}^{\prime n+1}=1, \quad z_{-n+1}^{\prime}=-z_{+}^{\prime a} z_{-a}^{\prime}
$$

Substituting the new coordinates (D.10) into the Kähler potential (D.1) and performing the gauge fixing gives the Kähler potential for the metric on $\mathcal{H}^{(2 n)}$ :

$$
\chi_{\mathcal{H}}\left(z_{+}^{a}, \bar{z}_{+}^{a}, z_{-a}, \bar{z}_{-a}\right)=2 \chi_{+} \chi_{-} .
$$

Here we introduced

$$
\chi_{+}:=\left(\eta_{I \bar{J}} z_{+}^{\prime I} \bar{z}_{+}^{\prime \bar{J}}\right)^{1 / 2}, \quad \chi_{-}:=\left(\eta^{I \bar{J}} z_{-I}^{\prime} \bar{z}_{-\bar{J}}^{\prime}\right)^{1 / 2}
$$


where it is understood that we have performed the gauge fixing (D.12).

In view of the later steps in the construction we also calculate $\omega_{\mathcal{H}}^{+}$. Substituting the primed coordinates into (D.3) and performing the gauge fixing gives

$$
\omega_{\mathcal{H}}^{+}=\mathrm{d} z_{+}^{\prime a} \wedge \mathrm{d} z_{-a}^{\prime}
$$

\section{The superconformal quotient: Going to twistor space $\mathcal{Z}^{(2 n-1)}$}

We now descend to the twistor space $\mathcal{Z}^{(2 n-1)}$. Here we follow [40] and introduce the coordinates

$$
z_{-i}^{\prime}=\mathrm{e}^{2 z} u_{i}, \quad z_{-n}^{\prime}=\mathrm{e}^{2 z}, \quad i=1, \ldots, n-1 .
$$

We next single out another coordinate, $\zeta$, which will be gauged when going to $X(n-1)$. To this end, we substitute the coordinates (D.16) into $\omega_{\mathcal{H}}^{+}$given in eq. (D.15):

$$
\omega_{\mathcal{Z}}^{+}=\mathrm{e}^{2 z}\left(2 \mathrm{~d} z_{+}^{\prime n}+2 u_{i} \mathrm{~d} z_{+}^{\prime i}\right) \wedge \mathrm{d} z+\mathrm{e}^{2 z} \mathrm{~d} z_{+}^{\prime i} \wedge \mathrm{d} u_{i}
$$

Following the general construction of the superconformal quotient, the components of this 2-form should be compared to

$$
\Omega_{\tilde{a} \tilde{b}}^{+}\left(u, z, z_{+}^{\prime}\right)=\mathrm{e}^{2 z}\left[\begin{array}{cc}
\omega_{\tilde{\imath} \tilde{\jmath}}\left(u, z_{+}^{\prime}\right) & X_{\tilde{\imath}}\left(u, z_{+}^{\prime}\right) \\
-X_{\tilde{\jmath}}\left(u, z_{+}^{\prime}\right) & 0
\end{array}\right] .
$$

From this comparison, we obtain the explicit form of $X_{\tilde{\imath}}$ :

$$
X_{\tilde{\imath}}=[\underbrace{0, \ldots, 0}_{(n-1) \text { times }}, 2 u_{1}, \ldots, 2 u_{n-1}, 2]^{\mathrm{T}} \text {. }
$$

We then determine $\zeta$ by first finding a $Y^{\tilde{\imath}}$, subject to $X_{\tilde{\imath}} Y^{\tilde{\imath}}=1$ and independent of the coordinates $z_{+}^{\prime a}, u_{i}, z$. The coordinate $\zeta$ is obtained as the solution of the 
differential equation

$$
Y^{\tilde{\imath}} \frac{\partial}{\partial z^{\tilde{\imath}}}=\frac{\partial}{\partial \zeta}
$$

Choosing $Y^{\tilde{\imath}}=[0, \ldots, 0,1 / 2]$, which is natural but not unique, we find $\zeta=2 z_{+}^{\prime}{ }^{n}$. This motivates to introduce

$$
z_{+}^{\prime i}=v^{i}, \quad z_{+}^{\prime n}=\frac{1}{2} \zeta
$$

The $v^{i}, u_{i}, \zeta$ and their complex conjugates then provide coordinates on the twistor space $\mathcal{Z}^{(2 n-1)}$. In order to obtain the Kähler potential of $\mathcal{Z}^{(2 n-1)}$ we first substitute these new coordinates into $\chi_{+}$and $\chi_{-}$:

$$
\begin{aligned}
& \chi_{+}=\left(1+\eta_{i \bar{\jmath}} v^{i} \bar{v}^{\bar{\jmath}}+\frac{1}{4} \zeta \bar{\zeta}\right)^{1 / 2} \\
& \chi_{-}=\mathrm{e}^{z+\bar{z}}\left(1+\eta^{i \bar{\jmath}} u_{i} \bar{u}_{\bar{\jmath}}+\left(v^{i} u_{i}+\frac{1}{2} \zeta\right)\left(\bar{v}^{\bar{\imath}} \bar{u}_{\bar{\imath}}+\frac{1}{2} \bar{\zeta}\right)\right)^{1 / 2}
\end{aligned}
$$

The Kähler potential of $\mathcal{Z}^{(2 n-1)}, K(v, u, \zeta, \bar{v}, \bar{u}, \bar{\zeta})$, can be deduced by comparing $\chi_{\mathcal{H}}$ given in (D.13) to the following expression:

$$
\chi_{\mathcal{H}}=\mathrm{e}^{z+\bar{z}+K(v, u, \zeta, \bar{v}, \bar{u}, \bar{\zeta})} .
$$

From this we read off

$$
K(v, u, \zeta, \bar{v}, \bar{u}, \bar{\zeta})=\ln \left(\chi_{+}\right)+\ln \left(\chi_{-}\right)+\ln (2)
$$

where $\chi_{+}$and $\chi_{-}$are taken at $z=0$.

In order to calculate the $S U(2)$ compensators appearing in the construction of the metric of $X(n-1)$, we also need $\omega_{\mathcal{Z}}^{+}$in terms of the coordinates $v^{i}, u_{i}, z, \zeta$. By substituting these coordinates into (D.17) we obtain

$$
\omega_{\mathcal{Z}}^{+}=\mathrm{e}^{2 z}\left(\mathrm{~d} \zeta+2 u_{i} \mathrm{~d} v^{i}\right) \wedge \mathrm{d} z+\mathrm{e}^{2 z} \mathrm{~d} v^{i} \wedge \mathrm{d} u_{i}
$$




\section{The superconformal quotient: The metric on $X(n-1)$}

We now descend to the quaternion-Kähler space $X(n-1)$ by setting $\zeta=0$. The Kähler potential $K$ becomes

$$
K(u, v, 0, \bar{u}, \bar{v}, 0)=\frac{1}{2} \ln \left(\phi_{+}\right)+\frac{1}{2} \ln \left(\phi_{-}\right)+\ln (2)
$$

where we introduced

$$
\phi_{+}:=1+\eta_{i \bar{\jmath}} v^{i} \bar{v}^{\bar{\jmath}}, \quad \phi_{-}:=1+\eta^{i \bar{\jmath}} u_{i} \bar{u}_{\bar{\jmath}}+\left(v^{i} u_{i}\right)\left(\bar{v}^{\bar{\imath}} \bar{u}_{\bar{\imath}}\right)
$$

However, since $\zeta$ is not parallel to the Killing vector $k^{+}$given in (3.1), the condition $\zeta=0$ is not preserved. In order to obtain the metric $G_{\alpha \bar{\beta}}$ on $X(n-1)$ we need to include an additional compensating transformation. Explicitly we have

$$
G_{\alpha \bar{\beta}}=K_{\alpha \bar{\beta}}-\mathrm{e}^{-2 K} X_{\alpha} \bar{X}_{\bar{\beta}}
$$

where $K_{\alpha \bar{\beta}}$ is the Kähler metric obtained from (D.26) and $\alpha=1, \ldots, 2 n-2$ enumerates the coordinates $\left\{v^{1}, \ldots, v^{n-1}, u_{1}, \ldots, u_{n-1}\right\}$. In order to determine the explicit from of the $X_{\alpha}$ appearing in the compensating transformation, we compare the components of $\omega_{\mathcal{Z}}^{+}$given in (D.25) to the general form of $\omega_{\mathcal{Z}}^{+}$given in [40]:

$$
\Omega_{a b}^{+}=\mathrm{e}^{2 z}\left[\begin{array}{ccc}
\omega_{\alpha \beta}(v, u) & 0 & X_{\alpha}(v, u) \\
0 & 0 & 1 \\
-X_{\beta}(v, u) & -1 & 0
\end{array}\right]
$$

From this we read off

$$
X_{\alpha}=[2 u_{1}, \ldots, 2 u_{n-1}, \underbrace{0, \ldots, 0}_{(n-1) \text { times }}]^{\mathrm{T}} .
$$


Having all these ingredients at hand, we can now write down the metric (D.28) explicitly. Arranging our indices as $X, Y=\left\{v^{i}, \bar{v}^{\bar{\imath}}, u_{i}, \bar{u}_{\bar{\imath}}\right\}$ the components of $G_{\alpha \bar{\beta}}$ can be read off from the following matrix:

$$
G_{X Y}=\left[\begin{array}{cccc}
0 & G_{v \bar{v}} & 0 & G_{v \bar{u}} \\
G_{\bar{v} v} & 0 & G_{\bar{v} u} & 0 \\
0 & G_{u \bar{v}} & 0 & G_{u \bar{u}} \\
G_{\bar{u} v} & 0 & G_{\bar{u} u} & 0
\end{array}\right]
$$

The entries of this matrix are given by

$$
\begin{aligned}
G_{u_{i} \bar{u}_{\bar{\jmath}}}= & \frac{1}{2 \phi_{-}}\left(\eta^{i \bar{\jmath}}+\bar{v}^{\bar{\jmath}} v^{i}\right)-\frac{1}{2 \phi_{-}^{2}}\left(\eta^{\bar{\jmath} l} u_{l}+\bar{v}^{\bar{\jmath}}\left(v^{l} u_{l}\right)\right)\left(\eta^{i \bar{l}} \bar{u}_{\bar{l}}+v^{i}\left(\bar{v}^{\bar{l}} \bar{u}_{\bar{l}}\right)\right) \\
G_{\bar{v}^{\bar{\imath}} u_{j}}= & \frac{1}{2 \phi_{-}^{2}}\left(\bar{u}_{\bar{\imath}} v^{j}\left(1+\eta^{k \bar{l}} u_{k} \bar{u}_{\bar{l}}\right)-\bar{u}_{\bar{\imath}} \eta^{j \bar{l}} \bar{u}_{\bar{l}}\left(v^{l} u_{l}\right)\right) \\
G_{v^{i} \bar{v}^{\bar{\jmath}}}= & \frac{1}{2 \phi_{+}} \eta_{i \bar{\jmath}}-\frac{1}{2 \phi_{+}^{2}}\left(\eta_{i \bar{l}} \bar{v}^{\bar{l}}\right)\left(\eta_{\bar{\jmath} l} v^{l}\right)-\frac{1}{\phi_{+} \phi_{-}} u_{i} \bar{u}_{\bar{\jmath}} \\
& +\frac{1}{2 \phi_{-}} u_{i} \bar{u}_{\bar{\jmath}}-\frac{1}{2 \phi_{-}^{2}} u_{i} \bar{u}_{\bar{\jmath}}\left(v^{l} u_{l}\right)\left(\bar{v}^{\bar{l}} \bar{u}_{\bar{l}}\right)
\end{aligned}
$$

The other non-vanishing entries of the matrix can be obtained from the condition that $G_{X Y}$ is hermitian with respect to $J^{3}$. This result establishes that the family of metrics (3.2) are indeed metrics on $X(n-1)$.

\section{D.2 The isometries of the Wolf spaces $X(n-1)$}

After obtaining the metric on $X(n-1)$, we will now derive the second ingredient needed in the construction of the LEEA and derive the Killing vectors and moment maps of the unitary Wolf spaces. We follow the calculation of [41] and extend these results.

The Killing vectors of flat $\mathbb{C}^{(2 n+2)}$ are given in (D.6). In order to find the Killing vectors on the hyper-Kähler cone $\mathcal{H}^{(2 n)}$ we perform a coordinate transformation to 
the primed coordinates (D.10). The resulting Killing vectors read:

$$
k_{+}^{\prime a}=i t_{I}^{a} z_{+}^{\prime I}-i z_{+}^{\prime a} t_{I}^{n+1} z_{+}^{\prime I}, \quad k_{-a}^{\prime}=i z_{-a}^{\prime} t_{I}^{n+1} z_{+}^{\prime I}-i t_{a}^{I} z_{-I}^{\prime}
$$

Here we have implicitly performed the gauge fixing (D.12).

To obtain the Killing vectors on $X(n-1)$ we first transform (D.33) into the coordinates $v^{i}, u_{i}, z, \zeta$ given by

$$
z_{+}^{\prime i}=v^{i}, \quad z_{+}^{\prime n}=\frac{1}{2} \zeta, \quad z_{-i}^{\prime}=\mathrm{e}^{2 z} u_{i}, \quad z_{-n}^{\prime}=\mathrm{e}^{2 z}
$$

Fixing $\zeta=0$, the resulting vectors $k^{\alpha}$ read:

$$
\begin{aligned}
k^{v^{i}} & =i t_{j}^{i} v^{j}+i t_{n+1}^{i}-i v^{i} t_{j}^{n+1} v^{j}-i v^{i} t_{n+1}^{n+1}, \\
k^{\zeta} & =2 i\left(t_{i}^{n} v^{i}+t_{n+1}^{n}\right) \\
k_{z} & =\frac{i}{2}\left(t_{i}^{n+1} v^{i}+t_{n+1}^{n+1}-t_{n}^{i} u_{i}-t_{n}^{n}+t_{n}^{n+1} v^{i} u_{i}\right), \\
k_{u_{i}} & =i u_{i}\left(t_{j}^{n+1} v^{j}+t_{n+1}^{n+1}\right)-i t_{i}^{j} u_{j}-i t_{i}^{n}+i t_{i}^{n+1}\left(v^{j} u_{j}\right)-2 u_{i} k_{z} .
\end{aligned}
$$

However, these vectors do not preserve the gauge $\zeta=0$. In order to get the Killing vectors $\hat{k}^{\alpha}$ on $X(n-1)$ we have to implement an additional compensating transformation [41]:

$$
\hat{k}^{\alpha}=k^{\alpha}-\frac{X^{\alpha}}{X^{\zeta}} k^{\zeta}
$$

According to [40], $X^{\alpha}, X^{\zeta}$ can be determined from the equations

$$
X^{\alpha}=\left(\hat{\omega}^{\alpha \beta} K_{\beta}+Z^{\alpha} K_{\zeta}\right) \mathrm{e}^{2 K}, \quad X^{\zeta}=\left(1-Z^{\alpha} K_{\alpha}\right) \mathrm{e}^{2 K}
$$

with $\hat{\omega}^{\alpha \beta}$ and $Z^{\alpha}$ given by

$$
\hat{\omega}^{\alpha \gamma} \omega_{\gamma \beta}=-\delta_{\beta}^{\alpha}, \quad Z^{\alpha}=-\hat{\omega}^{\alpha \beta} X_{\beta} .
$$


Here $K$ is the Kähler potential (D.24), $K_{\alpha}$ and $K_{\zeta}$ denote its derivative with respect to $v^{i}, u_{i}$ and $\zeta$, respectively, and $X_{\alpha}$ is given in (D.30). The $\omega_{\alpha \beta}$ is determined by comparing $\omega_{\mathcal{Z}}^{+}$given in (D.25) with the general expression (D.29) and $\hat{\omega}^{\alpha \beta}$ is obtained from eq. (D.38). Explicitly, we find

$$
\omega_{\alpha \beta}=\left[\begin{array}{cc}
0 & \mathbb{1} \\
-\mathbb{1} & 0
\end{array}\right], \quad \hat{\omega}^{\alpha \beta}=\left[\begin{array}{cc}
0 & \mathbb{1} \\
-\mathbb{1} & 0
\end{array}\right]
$$

where $\mathbb{1}$ denotes the $n$-1-dimensional unit matrix. Substituting $X_{\alpha}$ into (D.38) gives

$$
Z^{\alpha}=[\underbrace{0, \ldots, 0}_{n-1 \text { times }}, 2 u_{1}, \ldots, 2 u_{n-1}]^{\mathrm{T}} .
$$

With these results at hand, it is now straightforward to write down the explicit form of the compensating transformation appearing in (D.36):

$$
\frac{X^{v^{i}}}{X^{\zeta}}=\frac{1}{2}\left(\eta^{i \bar{\jmath}} \bar{u}_{\bar{\jmath}}+v^{i}\left(\bar{v}^{\bar{\jmath}} \bar{u}_{\bar{\jmath}}\right)\right), \quad \frac{X^{u_{i}}}{X^{\zeta}}=-\frac{\phi_{-}}{2 \phi_{+}} \eta_{i \bar{\jmath}} \bar{v}^{\bar{\jmath}} .
$$

The Killing vectors of $X(n-1)$ then read:

$$
\begin{gathered}
\hat{k}^{v^{i}}=i t^{i}{ }_{j} v^{j}+i t^{i}{ }_{n+1}-i v^{i} t_{j}^{n+1} v^{j}-i v^{i} t_{n+1}^{n+1}-\frac{k^{\zeta}}{2}\left(\eta^{i \bar{\jmath}} \bar{u}_{\bar{\jmath}}+v^{i} \bar{v}^{\bar{k}} \bar{u}_{\bar{k}}\right) \\
\hat{k}^{u_{i}}=i u_{i}\left(t^{n+1} v_{j}^{j}+t_{n+1}^{n+1}\right)-i t^{j}{ }_{i} u_{j}-i t_{i}^{n}+i t_{i}^{n+1}\left(v^{j} u_{j}\right) \\
-2 u_{i} k_{z}+\frac{\phi_{-}}{2 \phi_{+}} k^{\zeta} \eta_{i \bar{\jmath}} \bar{v}^{\bar{\jmath}}
\end{gathered}
$$

Here $k^{\zeta}$ and $k_{z}$ are given in (D.35).

We will now derive the moment maps associated with these Killing vectors, starting from the moment maps on flat $\mathbb{C}^{(2 n+2)}$ given in (D.8). Rewriting them in 
terms of the primed coordinates, the corresponding moment maps on $\mathcal{H}^{(2 n)}$ are:

$$
\begin{aligned}
& \mu^{3}=-\frac{2}{\chi_{(2 n)}}\left(\chi_{-}^{2} \bar{z}_{+}^{\prime \bar{I}} \eta_{\bar{I} J} t_{K}^{J} z_{+}^{\prime K}-\chi_{+}^{2} z_{-K}^{\prime} t_{J}^{K} \eta^{J \bar{I}} \bar{z}_{-\bar{I}}^{\prime}\right) \\
& \mu^{+}=-i z_{-I}^{\prime} t_{J}^{I} z_{+}^{J}, \quad \mu^{-}=\bar{\mu}^{+} \text {. }
\end{aligned}
$$

Again, it is understood that these expressions implicitly contain the gauge fixing (D.12).

In [41] it was found that the moment maps on the hyper-Kähler cone, $\mu^{r}$, and the moment maps on the underlying quaternion-Kähler manifold, $\hat{\mu}^{r}$, are related by

$$
\mu^{3}=\chi_{(2 n)} \hat{\mu}^{3}, \quad \mu^{+}=\mathrm{e}^{z-\bar{z}} \chi_{(2 n)} \hat{\mu}^{+}, \quad \hat{\mu}^{-}=\overline{\hat{\mu}}^{+} .
$$

Substituting in the coordinate transformation (D.34) and gauging $\zeta=0$, we obtain the following expression for the moment maps on $X(n-1)$ :

$$
\begin{aligned}
& \hat{\mu}^{3}=-\frac{1}{2 \phi_{+}}\left\{\bar{v}^{\bar{\imath}} \eta_{\bar{\imath} j} t_{k}^{j} v^{k}+t_{i}^{n+1} v^{i}+\bar{v}^{\bar{\jmath}} \eta_{\bar{\jmath} i} t_{n+1}^{i}+t_{n+1}^{n+1}\right\} \\
&+\frac{1}{2 \phi_{-}}\left\{u_{i} t^{i}{ }_{j} \eta^{j \bar{k}} \bar{u}_{\bar{k}}+u_{i} t_{n}^{i}+t^{n}{ }_{i} \eta^{i \bar{\jmath}} \bar{u}_{\bar{\jmath}}+t^{n}{ }_{n}-\left(u_{i} t^{i}{ }_{n+1}+t^{n}{ }_{n+1}\right)\left(\bar{u}_{\bar{\imath}} \bar{v}^{\bar{v}}\right)\right. \\
&\left.-\left(t^{n+1}{ }_{i} \eta^{i \bar{\jmath}} \bar{u}_{\bar{\jmath}}+t^{n+1}{ }_{n}\right)\left(u_{k} v^{k}\right)+t^{n+1}{ }_{n+1}\left(u_{i} v^{i}\right)\left(\bar{u}_{\bar{\jmath}} \bar{v}^{\bar{\jmath}}\right)\right\} \\
& \hat{\mu}^{+}=-\frac{i}{2 \phi_{+}^{1 / 2} \phi_{-}^{1 / 2}}\left\{u_{i} t^{i}{ }_{j} v^{j}+t^{n}{ }_{j} v^{j}-t^{n+1}{ }_{i} v^{i}\left(u_{j} v^{j}\right)+u_{i} t^{i}{ }_{n+1}\right. \\
&\left.+t_{n+1}^{n}-t_{n+1}^{n+1}\left(u_{i} v^{i}\right)\right\} .
\end{aligned}
$$

Together with eq. (D.42) this result completes the derivation of the formulae stated in subsection 3.1 . 


\section{E Initial values for numerical solutions}

\begin{tabular}{cccccccccc}
\hline \hline Traj. & $U$ & $\dot{U}$ & $W$ & $\dot{W}$ & $V$ & $\dot{V}$ & $\alpha$ & $\dot{\alpha}$ & $\beta$ \\
\hline $\mathrm{a}$ & 0.6 & -0.1 & 0.2 & 0.1 & 1 & 0.1 & 0 & 0.1 & 0 \\
$\mathrm{~b}$ & 0.8 & -0.1 & 0.5 & 0.1 & 1 & 0.1 & 0 & 0.1 & 0 \\
$\mathrm{c}$ & 0.85 & -0.1 & 0.7 & 0.1 & 1 & 0 & 0 & -0.2 & 0 \\
$\mathrm{~d}$ & 0.1 & -0.1 & 0.8 & 0.1 & 1 & 0.2 & 0 & 0.2 & 0 \\
$\mathrm{e}$ & 0.1 & -0.1 & 1.45 & 0.1 & 1 & 0.2 & 0 & 0.1 & 0 \\
$\mathrm{f}$ & 0.7 & -0.1 & 1.5 & 0.1 & 1 & -0.2 & 0 & -0.05 & 0 \\
$\mathrm{~L}$ & 0.8 & -0.1 & 1 & 0 & 1 & -0.2 & 0 & 0.1 & 0 \\
\hline \hline
\end{tabular}

Table 2: Initial conditions for the numerical solutions of the Out-picture equations of motion discussed in subsection 6.2 .

\begin{tabular}{ccccccccccc}
\hline \hline Traj. & $U$ & $\dot{U}$ & $W$ & $\dot{W}$ & $Q_{u}$ & $\dot{Q}_{u}$ & $Q_{v}$ & $q_{u}$ & $q_{v}$ & $\dot{\alpha}$ \\
\hline $\mathrm{b}^{\prime}$ & 0.8 & -0.1 & 0.5 & 0.1 & 0 & -0.05 & 0 & 0.1 & 0 & 0.1 \\
$\mathrm{i}$ & 0.3 & 0 & 3 & 0 & 0 & 0 & 0 & 0.07 & 0.02 & 0.1 \\
$\mathrm{~g}$ & 0.6 & 0 & 0.6 & 0 & -0.2 & 0 & 0.5 & -0.05 & 0.3 & 0.2 \\
\hline \hline
\end{tabular}

Table 3: Initial conditions for the numerical solutions of the In-picture equations of motion discussed in subsection 7.3. Additionally we take $\dot{q}_{u}=\dot{q}_{v}=\dot{Q}_{v}=0, \alpha=\beta=0$ for all three solutions.

\begin{tabular}{cccccccc}
\hline \hline Traj. & $\phi$ & $\dot{\phi}$ & $q$ & $\dot{q}$ & $\alpha$ & $\dot{\alpha}$ & $\beta$ \\
\hline $\mathrm{cf}$ & 0 & 0.09 & 0.65 & 0 & 0 & 0.1 & 0 \\
\hline \hline
\end{tabular}

Table 4: Initial conditions for the numerical solution undergoing a dynamical conifold transition presented in subsection 8.2. Additionally we take $\alpha=\beta=0$. The initial values of the other hypermultiplet scalars fields follow from restricting the hypermultiplet scalar manifold to the subspace (8.1). 


\section{Zusammenfassung}

Unser derzeitiges Verständnis der fundamentalen Wechselwirkungen der Natur basiert auf dem Standardmodel der Elementarteilchenphysik und der Allgemeinen Relativitätstheorie. Diese beiden Theorien lassen sich im Rahmen von zehn-dimensionalen Superstringtheorien konzeptionell vereinheitlichen. Darüber hinaus wird vermutet, dass sich alle konsistenten Superstringtheorien in einer elf-dimensionalen fundamentalen Theorie, der M-Theorie, vereinigen lassen. Neben einem masselosen Sektor, der durch die elf-dimensionale Supergravitation gegeben ist, enthält das MTheorie-Spektrum ausgedehnte Objekte, welche als geladene, stationäre Lösungen der Bewegungsgleichungen auftreten, sogenannte M2- und M5-Branen. Um die elfdimensionale Raumzeit der M-Theorie (Mikrophysik) mit unserem vier-dimensionalen Universum (Makrophysik) zu verbinden, kompaktifiziert man die Extradimensionen auf einer kompakten Mannigfaltigkeit, deren Längenskalen unterhalb der in Beschleunigerexperimenten untersuchten Abstände liegen. Die Makrophysik wird dabei durch eine effektive Niederenergiewirkung beschrieben, die nur die leichten Moden der mikroskopischen Theorie beinhaltet.

In diesem Rahmen beschäftigt sich die vorliegende Arbeit mit effektiven Niederenergiewirkungen für Calabi-Yau (CY) Kompaktifizierungen von M-Theorie, die bestimmte Windungszustände von M2-Branen explizit berücksichtigen. Die resultierenden fünf-dimensionalen effektiven Wirkungen bieten gegenüber einer direkten Kompaktifizierung nach vier Dimensionen den Vorteil, dass der Vektormultipletsektor exakt behandelt werden kann. Zusätzlich gleicht ihr Hypermultipletsektor dem von CY Kompaktifizierungen der Typ IIA Stringtheorie, so dass die hier gefundenen Ergebnisse auf vier-dimensionale effektive Wirkungen übertragen werden können.

Ohne Hinzunahme von Windungszuständen ist die gewöhnliche effektive Wirkung (GEW) einer M-Theorie-Kompaktifizierung auf einer glatten CY Mannigfaltigkeit $X$ mit Hodgezahlen $h^{p, q}(X)$ eine $\mathcal{N}=2, D=5$ Supergravitationswirkung 
mit $n_{V}=h^{1,1}(X)-1$ abelschen Vektor- und $n_{N}=h^{1,2}(X)+1$ neutralen Hypermultiplets. Die Kopplungskonstanten der GEW sind durch die geometrischen Daten von $X$ vollständig bestimmt. Der Moduli-Raum der Vacua der Wirkung entspricht den Deformationen der CY Mannigfaltigkeit und der M-Theorie Hintergrundfelder. Dieser Raum enthält spezielle Punkte an denen holomorphe Untermannigfaltigkeiten von $X$ kollabieren, wodurch $X$ zu einem singulären CY Raum $\hat{X}$ wird. Lassen sich die so entstehenden Singularitäten auf zwei (oder mehr) topologisch unterschiedliche Weisen glätten spricht man von einem topologischen Phasenübergang.

Die GEW wird während eines solchen Phasenübergangs unstetig oder singulär. Im Rahmen der vollständigen M-Theorie-Beschreibung ist dieses Verhalten jedoch ein Artefakt der GEW, welches durch Ignorieren der auf den kollabierenden Untermannigfaltigkeiten lokalisierten Windungszuständen entsteht. Die Masse solcher Zustände ist proportional zum Volumen der schrumpfenden Untermannigfaltigkeit, so dass an den singulären Punkten zusätzliche masselose Moden (Übergangszustände) auftreten. Die Singularitäten der GEW können dann durch das unberechtigte Ausintegrieren der zusätzlichen masselosen Moden erklärt werden.

Diese Behandlung der Übergangszustände ist jedoch unbefriedigend, da eine vollständige Beschreibung der Makrophysik in der Nähe des Phasenübergangs alle leichten Moden explizit berücksichtigen sollte. Daher befaßt sich der erste Teil der vorliegenden Arbeit mit der Konstruktion von erweiterten effektiven Wirkungen (EEW), die solche Moden beinhalten. Hierbei konzentrieren wir uns auf eine bestimmte Klasse von singulären CY Räumen $\hat{X}$, nämlich solche mit $N$ Doppelpunkten. Diese entstehen durch Deformationen von $X$ in denen $N$ holomorphe Kurven $\mathcal{C}_{i}, i=1, \ldots, N,\left(\operatorname{dim}_{\mathbb{C}}\left(\mathcal{C}_{i}\right)=1\right)$ zu Punkten schrumpfen. In diesem Fall gibt es zwei topologische Phasenübergänge, die glatte, aber topologisch unterschiedliche CY Mannigfaltigkeiten $X$ und $\tilde{X}$ verbinden:

1. Bei einem Flopübergang liegen alle holomorphen Kurven $\mathcal{C}_{i}$ in der gleichen Homologieklasse $C \in H_{2}(X, \mathbb{Z})$. Durch das Schrumpfen der $\mathcal{C}_{i}$ zu Doppel- 
punkten und anschließender Expansion zu holomorphen Kurven $\tilde{\mathcal{C}}_{i}$ in der Homologieklasse $\tilde{C}=-C$ gelangt man zu einer topologisch inäquivalenten $\mathrm{CY}$ Mannigfaltigkeit $\tilde{X}$ mit gleichen Hodge- aber anderen Tripelschnittzahlen.

2. Erfüllen die $N$ kollabierenden Kurven $N>r>0$ Homologierelationen, gibt es zusätzlich die Möglichkeit von Conifoldübergängen. Hier können die resultierenden Doppelpunkte auch durch das Einfügen von drei-Sphären geglättet werden. Die Hodgezahlen der neuen glatten CY Mannigfaltigkeit $\tilde{X}$ sind dann

$$
h^{1,1}(\tilde{X})=h^{1,1}(X)-(N-r), \quad h^{1,2}(\tilde{X})=h^{1,2}(X)-r
$$

so dass sich die Eulerzahlen der beteiligten CY Mannigfaltigkeiten um $\chi(\tilde{X})=$ $\chi(X)-2 N$ unterscheiden.

Die zusätzlichen leichten Moden am Phasenübergäng ergeben sich aus den auf den Kurven $\mathcal{C}_{i}$ lokalisierten Windungszuständen von M2-Branen. In der feldtheoretischen Beschreibung entsprechen diese Zustände $N$ geladenen Hypermultiplets, deren Masse proportional zum Volumen der Kurven $\mathcal{C}_{i}$ ist.

Unser Ziel ist es, diese zusätzlichen Hypermultiplets in eine EEW zu integrieren. Aufgrund der zusätzlichen geladenen Zustände ist die EEW dann eine geeichte $\mathcal{N}=2, D=5$ Supergravitationswirkung mit $n_{V}$ Vektor- und $n_{H}=n_{N}+N$ Hypermultiplets. Bei der expliziten Konstruktion der Wirkungen kombinieren wir die mikroskopische M-Theorie-Beschreibung der Übergänge mit der Form der allgemeinsten geeichten $\mathcal{N}=2, D=5$ Supergravitationswirkung.

Dabei stellt sich heraus, dass der Vektormultipletsektor der EEW exakt bestimmt werden kann. In der GEW wird dieser Sektor vollständig durch die Tripelschnittzahlen der CY Mannigfaltigkeit $X$ festgelegt. Aufbauend auf einem Resultat von E. Witten für die durch Ausintegrieren der geladenen Hypermultiplets entstehenden Schwellenkorrekturen, ergibt sich eine explizite Relation zwischen den Tripelschnittzahlen von $X$ und dem Vektormultipletsektor der EEW. 
Im Hypermultipletsektor ist die Situation jedoch schwieriger. Die $n_{H}$ Hypermultiplets der EEW parametrisieren eine quaternion-Kähler Mannigfaltigkeit. Diese ist im Allgemeinen weder Kähler noch komplex, trägt jedoch eine quaternionische Struktur. Da Rechnungen mit solchen Mannigfaltigkeiten sehr kompliziert sind, leiten wir die quaternion-Kähler Mannigfaltigkeiten der EEW nicht aus der Kompaktifizierung ab, sondern approximieren den Hypermultipletsektor durch die Familie der unitären Wolfräume,

$$
X(N+1)=\frac{U(N+1,2)}{U(N+1) \times U(2)}
$$

für $N+1$ Hypermultiplets.

Die Konstruktion einer geeichten Supergravitationswirkung basierend auf diesen Mannigfaltigkeiten erfordert explizite Ausdrücke für die Metrik, Killingvektoren und Impulsabbildungen auf $X(N+1)$. Diese leiten wir mittels superkonformer Quotientenbildung aus den entsprechenden geometrischen Größen des Hyperkählerkegels über $X(N+1)$ her. Die verwendete Methode ist dabei nicht auf die Wolfräume beschränkt, sondern kann auf jede quaternion-Kähler Mannigfaltigkeit angewendet werden. Die so erhaltenen Resultate beschreiben den allgemeinsten auf $X(N+1)$ basierenden Hypermultipletsektor einer geeichten $\mathcal{N}=2$ Supergravitationswirkung in $3 \leq D \leq 6$ Dimensionen.

Aufbauend auf diesen Ergebnissen konstruieren wir zwei Familien von EEW mit allen zur Beschreibung eines Flop- oder Conifoldübergangs benötigten Eigenschaften. Die resultierenden EEW sind nach der Wahl der skalaren Mannigfaltigkeit im Hypermultipletsektor eindeutig durch die M-Theorie-Beschreibung bestimmt. Die Massen der Übergangszustände werden durch das skalare Potential der Wirkung kontrolliert und stimmen mit den Erwartungen aus der M-Theorie-Beschreibung überein. Weiter bilden die Minima des skalaren Potentials eine Familie supersymmetrischer Minkowski-Grundzustände, die den Moduli der CY Kompaktifizierungen auf $X$ und $\tilde{X}$ entsprechen. Im Gegensatz zur GEW sind die neuen EEW am topolo- 
gische Phasenübergang glatt, d.h. physikalische Größen entwickeln keine Unstetigkeiten oder Singularitäten am Übergangspunkt.

Der zweite Teil der Arbeit untersucht das Verhalten der Übergangszuständen in fünf-dimensionalen Kasner Kosmologien anhand spezifischer Beispiele. Dazu wählen wir die Raumzeit-Metrik als

$$
\mathrm{d} s^{2}=-\mathrm{d} t^{2}+e^{2 \alpha(t)} \mathrm{d} \vec{x}^{2}+e^{2 \beta(t)} \mathrm{d} y^{2}
$$

Die räumlichen Dimensionen sind dabei durch die drei Koordinaten $\vec{x}=\left(x^{1}, x^{2}, x^{3}\right)$ des $x$-Raums und einer Extradimension mit Koordinate $y$ gegeben. Obwohl fünfdimensionalen Kosmologien a priori keinen Vergleich mit den physikalischen Observablen des beobachtbaren Universums erlauben, ermöglicht dieser Ansatz eine dynamische Untersuchung der qualitativen Eigenschaften der Phasenübergänge.

Bei Verwendung der GEW, in der die Übergangszustände nicht explizit berücksichtigt werden, sind die physikalischen Observablen während eines Flopübergangs stetig. Conifoldübergänge hingegen können nicht dynamisch realisiert werden. Weiter schließen die Bewegungsgleichungen eine beschleunigte Expansion des $x$-Raum aus. Die Moduli besitzen typischerweise ein Weglaufverhalten, d.h. die Lösungskurven der Skalarfelder laufen durch die entsprechenden skalaren Mannigfaltigkeiten bis sie einen Rand erreichen.

Werden die Übergangszustände ebenfalls dynamisch behandelt, können sowohl Flop- als auch Conifoldübergänge dynamisch erfolgen und alle physikalischen Observablen sind am Übergangspunkt glatt. Dies ist insbesondere bei Conifoldübergängen bemerkenswert, da diese CY Kompaktifizierungen mit einer unterschiedlichen Anzahl von masselosen Vektor- und Hypermultiplets verbinden. Weiter hat das skalare Potential der EEW tiefgehende Konsequenzen für die Stabilisierung der Moduli und eine beschleunigte Expansion des $x$-Raums.

Bei Anregung aller leichten Zustände werden die Moduli dynamisch in der Übergangsregion eingeschlossen, d.h. die Skalarfelder oszillieren im Übergangsbereich. 
Daraus folgt, dass die "fast singulären" Mannigfaltigkeiten in der Nähe des Phasenübergangs dynamisch bevorzugt sind. Zusätzlich ermöglicht das skalare Potential eine beschleunigte Expansion des $x$-Raumes. Obwohl die Slow-Roll Bedingungen nicht erfüllt sind, ergeben sich typischerweise mehrere kurze Episoden beschleunigter Expansion, die zur Erklärung der heutigen, moderat beschleunigten Expansion des Universums interessant sein könnten.

Zusammenfassend zeigt die vorliegende Arbeit, dass Übergangszustände in Flopund Conifoldübergängen einen wichtigen Beitrag zur Stabilisierung der Form der internen Mannigfaltigkeit leisten können. Die hier konstruierten Potentiale bieten somit eine Alternative zu skalaren Potentialen aus hyperbolischen Kompaktifizierungen oder M-Theorie-Kompaktifizierungen mit Hintergrundflüssen. Im Rahmen eines zukünftigen Projekts wäre es interessant zu untersuchen, ob das Zusammenspiel von Übergangszuständen und Hintergrundflüssen oder Quantenkorrekturen im Hypermultipletsektor zu einer nachhaltigen beschleunigten Expansion des Universums führt. 


\section{Danksagung}

An dieser Stelle möchte ich all jenen danken, die auf direktem oder indirektem Weg zu der Entstehung dieser Arbeit beigetragen haben.

Mein ganz besonderer Dank gilt Dr. Thomas Mohaupt für die sehr gute Betreuung dieser Arbeit und seine Weitsicht beim Vorschlag des Themas. So entstand ein Großteil dieser Arbeit bevor Begriffe wie "the central region of moduli space" und "enhanced symmetry points (ESPs)" in der Literatur populär wurden. Es ist mir eine Ehre, an der Entwicklung dieses Gebietes mitarbeiten zu können.

Ein großes Dankeschön geht an den Rest "meiner" Stringnachwuchsgruppe, Dr. Laur Järv und Christoph Mayer für die gute Zusammenarbeit und viele interessante fachliche Diskussionen. Insbesondere danke ich Laur für viele Erklärungen zu den Feinheiten der englischen Grammatik und Syntax, die wesentlich zum Entstehen dieser Arbeit in ihrer jetzigen Form beigetragen haben. Weiter gilt mein Dank Dr. K. Behrndt, Prof. Dr. V. Cortés, Dr. G. Dall'Agata, Prof. Dr. B. de Wit, Prof. Dr. J. Louis, Prof. Dr. S. Vandoren und Prof. Dr. A. Van Proeyen die durch hilfreiche Kommentare zum Gelingen dieser Arbeit beigetragen haben. Ferner gilt mein Dank Prof. Dr. M. Reuter, der mir immer ein kompetenter Ansprechpartner war und mit vielen sehr guten Ratschlägen zur Seite gestanden hat.

Sehr genossen habe ich auch die vielen interessanten Gespräche und Diskussionen fachlicher und außerfachlicher Natur am Theoretisch-Physikalischen Institut Jena. Stellvertretend seien hier A. Kirchberg, J.D. Länge, Prof. Dr. G. Schäfer, Dr. T. Strobl und Prof. Dr. A. Wipf genannt. Für ihre Hilfe bei Computerproblemen jeglicher Art möchte ich mich weiter bei Dr. T. Heinzl, Dr. E. Wöhnert und Dr. M. Weiß bedanken. Ein besonderer Dank geht an Frau G. Ritter, die durch ihre freundliche und zuvorkommende Art sehr zum guten Betriebsklima und reibungslosen Arbeitsablauf am Institut beiträgt. 
Besonderer Dank gebührt auch "meiner" Diplom-Bibliothekarin, privaten Informationbrokerin und Freundin Christiane, für ihre Geduld beim Zuhören (insbesondere, wenn es mal wieder um Physik geht) und dem Organisieren schwierig zu bekommender Zeitschriftenartikel. Ihr verdanke ich es, dass ich neben meinem Büro noch viele andere schöne Seiten von Jena kennengelernt habe.

Nicht zu letzt danke ich meiner Familie und insbesondere meinen Eltern die mich immer in der bestmöglichen Weise unterstützt haben.

Abschließend danke ich dem Land Thüringen und der Studienstiftung des deutschen Volkes, die diese Arbeit durch ihre finanzielle Unterstützung ermöglicht haben. Weiter sei hier die DFG erwähnt, die durch Reisemittel dazu beigetragen hat, dass ich viele interessante Fachkonferenzen und Schulen im In- und Ausland besuchen konnte. 


\section{Ehrenwörtliche Erklärung}

Ich erkläre hiermit ehrenwörtlich, dass ich die vorliegende Arbeit selbständig, ohne unzulässige Hilfe Dritter und ohne Benutzung anderer als der angegebenen Hilfsmittel und Literatur angefertigt habe. Die aus anderen Quellen direkt oder indirekt übernommenen Daten und Konzepte sind unter Angabe der Quelle gekennzeichnet.

Niemand hat von mir unmittelbar oder mittelbar geldwerte Leistungen für Arbeiten erhalten, die im Zusammenhang mit dem Inhalt meiner Dissertation stehen. Insbesondere habe ich hierfür nicht die entgeltliche Hilfe von Vermittlungs- bzw. Beratungsdiensten in Anspruch genommen.

Die Arbeit wurde bisher weder im In- noch im Ausland in gleicher oder ähnlicher Form einer anderen Prüfungsbehörde vorgelegt.

Die geltende Prüfungsordnung der Physikalisch-Astronomischen Fakultät ist mir bekannt.

Ich versichere ehrenwörtlich, dass ich nach bestem Wissen die reine Wahrheit gesagt und nichts verschwiegen habe. 


\section{Lebenslauf}

$\begin{array}{ll}\text { Name } & \text { Saueressig } \\ \text { Vorname } & \text { Frank Simon } \\ \text { geboren } & \text { 7. September 1975 in Bad Kreuznach } \\ \text { Nationalität } & \text { deutsch }\end{array}$

\section{Schule}

$9.1982-8.1986$

$9.1986-8.1992$

$9.1992-6.1995$

22.6 .1995

\section{Zivildienst}

$8.1995-9.1996$

\section{Studium}

$10.1996-9.1999$

$10.1999-9.2000$

$10.2000-11.2001$

21.11.2001

\section{Promotion}

seit 12.2001
Astrid-Lindgren-Schule, Waldalgesheim

Rochus Realschule, Bingen

Technisches Gymnasium, BBS I, Mainz

Abitur (Note: 1.0)

ev. Kirchengemeinde Bingerbrück

Physikstudium, Johannes-Gutenberg Universität, Mainz

Integriertes Auslandsstudium:

University of Washington, Seattle

Diplomarbeit, Johannes-Gutenberg Universität, Mainz

"Renormierungsgruppenfluss der Quanten-Einstein-

Gravitation in der Einstein-Hilbert-Trunkierung

und nichtlokalen Erweiterungen"

Betreuer: Prof. Dr. M. Reuter

Physik Diplom (Note: Mit Auszeichnung bestanden)

Theoretisch-Physikalisches Institut

Friedrich-Schiller-Universität, Jena

Betreuer: Dr. habil. T. Mohaupt 